\title{
Distributed Coordination Control of Complex Multi-agent Networks with Dynamic Interaction Topologies
}

\author{
A Dissertation \\ Presented to \\ the faculty of the School of Engineering and Applied Science \\ University of Virginia
}

\author{
in partial fulfillment \\ of the requirements for the degree \\ Doctor of Philosophy
}

by

Shize Su

December 2017 
(c) 2017 Shize $\mathrm{Su}$ 


\title{
APPROVAL SHEET
}

\author{
This Dissertation \\ is submitted in partial fulfillment of the requirements \\ for the degree of \\ Doctor of Philosophy
}

Author Signature: Shize su

This Dissertation has been read and approved by the examining committee:

Advisor: Zongli Lin

Committee Member: Gang Tao

Committee Member: Peter Beling

Committee Member: Quanquan Gu

Committee Member: Scott Acton

Committee Member:

Accepted for the School of Engineering and Applied Science:

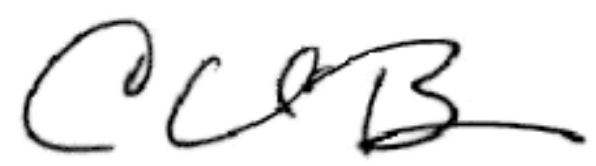

Craig H. Benson, School of Engineering and Applied Science

December 2017 


\section{Abstract}

Distributed coordination control of complex multi-agent networks has received significant attention over the past decades, due to its widely recognized advantages and potentials in many applications such as large scale sensor networks, robotic networks, power grid, distributed computing clouds, social networks and biological networks. Within the control theory community, the main research task in an engineering multi-agent network system is to design distributed control algorithms, which only use local neighborhood information, to achieve some specified global objectives.

Distributed consensus and synchronization are two important problems in complex engineering multi-agent networks. Consensus is concerned with reaching a network-wide agreement on some quantities of interest while each agent can only access local information. Synchronization defines the correlated-in-time behavior between different agents achieved by local interaction strategies. Synchronization control is also employed to achieve distributed coordinated tracking objectives in multi-agent networks.

A multi-agent network can be quite complex. The amount of agents could be huge, each agent might have complicated dynamics which might possibly be nonlinear and unknown, and the interaction topology among the agents could change over time. Regardless of the control algorithms employed, coordinated control of multi-agent networks relies heavily on interactions among agents, and for this reason the interaction topology plays an essential role in the development of the distributed coordination control algorithms as well as the analysis of their performance. Two major approaches, the common Lyapunov function approach and the multiple Lyapunov function approach, have been demonstrated to be effective in developing and analyzing control protocols for multi-agent networks with dynamic interaction topologies.

In this thesis, we investigate distributed coordination control problems of complex multi-agent networks with dynamic interaction topologies, which include three main parts summarized as follows.

i) In the first part of this thesis, we consider the distributed synchronization control problem for a multiagent network with $n$th order unknown nonlinear agent dynamics. We first establish the results for both an undirected time varying interaction topology and a directed time varying interaction topology. A standard assumption on the interaction topology connectivity in the literature on this type of problems 
is made, namely, the interaction topology is connected. Distributed synchronization control algorithms are developed and the desired synchronization performance of the multi-agent network system is proven.

ii) In the second part of this thesis, we address the distributed consensus control problem of a multiagent network with high order linear agent dynamics under a directed jointly connected interaction topology. This enriches the existing literature on multi-agent distributed consensus control under a jointly connected directed interaction topology, which was limited to very simple single integrator agent dynamics. The distributed control protocols are developed and the consensus is proven.

iii) In the final part of this thesis, we investigate the distributed coordinated tracking problem for a multi-agent network under a state dependent jointly connected dynamic interaction topology. In many applications such as in situations where information exchange among agents is conducted via equipped sensors, the interaction topology depends on agents' states such as the distance among agents, and cannot be simply assumed as a function of time. The distributed control protocols are developed and the coordinated tracking is proven. In addition, a new topology connectivity enhancing mechanism is proposed to help ensure coordinated tracking in real world implementations. In comparison with the existing topology connectivity preserving algorithms, our proposed topology connectivity enhancing mechanism is effective in maintaining both the initially existent topology edges and the newly formed topology edges, which enables us to relax the more restrictive requirement that the interaction topology is initially connected. 
To everyone who 's helped me succeed 


\section{Acknowledgments}

The past years at the University of Virginia has been an incredible journey for me. Not only have I learned about the engineering principles from books and lectures, but I have also learned from the life experiences of the people I have met and interacted with. I would like to use this opportunity to recognize the people who have supported and encouraged me throughout the graduate program.

First of all, I would give my sincere thanks to my advisor Professor Zongli Lin. I am greatly indebted to my mentor Professor Zongli Lin for his support throughout the graduate program. I am honored to have had the opportunity to grow as a member in the scientific community under his guidance.

Besides my advisor, I would also like to thank all the other members in my dissertation committee, Professor Gang Tao, Professor Peter Beling, Professor Quanquan Gu and Professor Scott Acton, for your valuable feedbacks to my research which helped me improve this work.

Special thanks to all my friends and colleagues whom I had the honor to meet and work together during my Ph.D. study.

I am greatly indebted to my family for always believing in me. Their encouragement and support have been invaluable during my growth as an engineer and a person. Finally, I am most grateful to my wife Xiali Wang for her everlasting love and support. I cannot find the words to describe my appreciation for everything that she had to sacrifice to walk this path with me, and all I can say is a very humble "thank you and I love you". 


\section{Contents}

Contents

List of Figures . . . . . . . . . . . . . . . . . . . . . . . . . vii

1 Introduction 1

1.1 Overview of Multi-agent Networks . . . . . . . . . . . . . . . . . . . 1

1.2 Distributed Consensus Control of Multi-agent Networks under Dynamic Interaction Topologies 2

1.3 Distributed Synchronization Control of Multi-agent Networks under Dynamic Interaction

Topologies . . . . . . . . . . . . . . . . . . . . . . . . 3

1.4 Distributed Coordinated Tracking of Multi-agent Networks under State Dependent Dynamic Interaction Topologies . . . . . . . . . . . . . . . . . . . . . . . . . 4 4

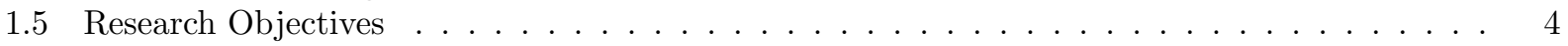

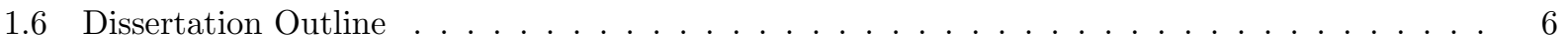

2 Fundamentals $\quad 7$

2.1 Graph Theory . . . . . . . . . . . . . . . . . . . . . . . . . . 7

2.2 Switched System Theory . . . . . . . . . . . . . . . . . . . . 8

3 Distributed Synchronization Control of Multi-agent Networks With Unknown Nonlinearities under Connected Dynamic Interaction Topologies 10

3.1 Problem Statement . . . . . . . . . . . . . . . . . . . . . . . . . . 10

3.2 Synchronization Control of $n$ th-order Systems with a Fixed Strongly-connected Directed Graph Communication Topology . . . . . . . . . . . . . . . . . . . . . . . . . . . 12

3.2.1 Control Protocols and the Closed-loop System Performance . . . . . . . . . . . . . . . 12

3.2.2 Theoretical Analysis . . . . . . . . . . . . . . . . . . . . . . . . . 14

3.3 Synchronization Control of $n$ th-order Systems with Switching Connected Undirected Graph Communication Topologies . . . . . . . . . . . . . . . . . . . . 16

3.3.1 Control Protocols and the Closed-loop System Performance . . . . . . . . . . . . . . . 17

3.3.2 Theoretical Analysis . . . . . . . . . . . . . . . . . . . . . . 18

3.4 Synchronization Control of $n$ th-order Systems with Switching Strongly-connected Directed Graph Communication Topologies . . . . . . . . . . . . . . . . . . . . . . 21

3.4.1 Control Protocols and the Closed-loop System Performance . . . . . . . . . . . . . . . 22

3.4 .2 Theoretical Analysis . . . . . . . . . . . . . . . . . . . . . . . . . 24

3.5 Simulation Results . . . . . . . . . . . . . . . . . . . . . . . 30

3.5.1 Fixed Strongly-connected Directed Graph Communication Topology . . . . . . . . . . 31

3.5.2 Switching Connected Undirected Graph Communication Topology . . . . . . . . . . . 39

3.5.3 Switching Strongly-connected Directed Graph Communication Topology . . . . . . . . 43

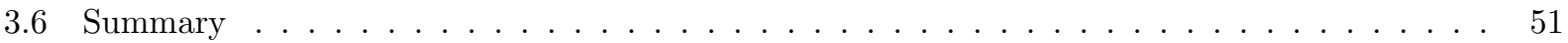

4 Distributed Consensus Control under Jointly Connected Dynamic Interaction Topologies $\mathbf{5 3}$

4.1 Problem Statement . . . . . . . . . . . . . . . . . . . . . . . . . . 53

4.2 Consensus Control Protocols and the Closed-loop System Performance Analysis . . . . . . . . 56

4.2.1 Consensus Control Protocols and the Closed-loop System Performance . . . . . . . . 56 
4.2 .2 Theoretical Analysis . . . . . . . . . . . . . . . . . . . . . . 57

4.2 .3 Discussion . . . . . . . . . . . . . . . . . . . . . . . . . 58

4.3 Simulation Results . . . . . . . . . . . . . . . . . . . . . . . . . 59

4.3.1 Consensus without a Leader . . . . . . . . . . . . . . . . . . . . . . 59

4.3.2 Consensus with a Stationary Leader . . . . . . . . . . . . . . . . . . . . . 62

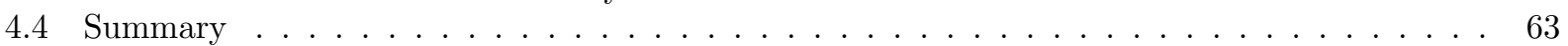

5 Distributed Coordinated Tracking under State Dependent Dynamic Interaction Topologies

5.1 Problem Statement . . . . . . . . . . . . . . . . . . . . . . . 65

5.2 Virtual Leader Tracking Control Protocols and the Closed-loop System Performance Analysis 68 5.2.1 Control Protocols and the Closed-loop System Performance Analysis with Virtual

Leader Velocity Information . . . . . . . . . . . . . . . . . . . . . . . . . . . 68

5.2.2 Control Protocols and the Closed-loop System Performance Analysis without Virtual Leader Velocity Information . . . . . . . . . . . . . . . . . . . . . . . . . . . . . . . 75

5.2.3 Connectivity Enhancing Coordinated Tracking Control Protocols and the Closed-loop System Performance Analysis . . . . . . . . . . . . . . . . . . . . . . . . 83

5.3 Simulation Results . . . . . . . . . . . . . . . . . . . . . . . . . . 87

5.3.1 Distributed Coordinated Tracking Control with Virtual Leader Velocity Information . 87

5.3.2 Distributed Coordinated Tracking Control without Virtual Leader Velocity Information 89

5.4 Summary . . . . . . . . . . . . . . . . . . . . . . . . . 94

6 Conclusions 


\section{List of Figures}

2.1 Two Lyapunov functions (blue solid thick lines correspond to active $V_{1}$, red dashed thick lines correspond to active $V_{2}$, black dashed thin lines correspond to inactive $V_{1}$, green dashed thin lines correspond to inactive $V_{2}$ ): a) left figure - continuous $V_{\sigma}$; b) right figure - discontinuous $V_{\sigma} 9$

3.1 A fixed digraph communication topology . . . . . . . . . . . . . . . . . . 31

3.2 Phase plot, fixed digraph topology, Case $1, \mathrm{c}=100 \ldots \ldots \ldots \ldots \ldots \ldots$

3.3 Tracking error plot, fixed digraph topology, Case $1, \mathrm{c}=100 \ldots \ldots \ldots \ldots$

3.4 RBFNN approximation, fixed digraph topology, Case $1, \mathrm{c}=100 \ldots \ldots \ldots \ldots$

3.5 Tracking error plot, fixed digraph topology, Case $2, c=20 \ldots \ldots \ldots \ldots$

3.6 Tracking error plot, fixed digraph topology, Case $2, \mathrm{c}=30 \ldots \ldots \ldots$

3.7 Tracking error plot, fixed digraph topology, Case $2, \mathrm{c}=60 \ldots \ldots \ldots \ldots$

3.8 Tracking error plot, fixed digraph topology, Case $2, \mathrm{c}=100 \ldots \ldots \ldots \ldots$

3.9 Phase plot, fixed digraph topology, Case 3 , $\mathrm{c}=60$, with RBFNN $\ldots \ldots \ldots \ldots$

3.10 Phase plot, fixed digraph topology, Case 3, $\mathrm{c}=60$, without RBFNN $\ldots \ldots \ldots$. . . . . . 37

3.11 Phase plot, fixed digraph topology, Case 3, $\mathrm{c}=200$, without RBFNN $\ldots \ldots \ldots$. . . . . 37

3.12 Tracking error plot, fixed digraph topology, Case 3 , $\mathrm{c}=60$, with RBFNN $\ldots \ldots \ldots$

3.13 Tracking error plot, fixed digraph topology, Case 3, $\mathrm{c}=60$, without RBFNN $\ldots \ldots$. . . . 38

3.14 Tracking error plot, fixed digraph topology, Case 3, $\mathrm{c}=200$, without RBFNN . . . . . . . . 39

3.15 A switching connected undirected communication topology . . . . . . . . . . . . . 40

3.16 Phase plot, switching undirected graph topology, $\mathrm{c}=60 \ldots \ldots \ldots \ldots$. . . . . . . . 41

3.17 Tracking error plot, switching undirected graph topology, $\mathrm{c}=60 \ldots \ldots$. . . . . . . . 42

3.18 RBFNN approximation, switching undirected graph topology, $c=60 \ldots \ldots$. . . . . . . 42

3.19 A switching strongly-connected directed graph communication topology . . . . . . . . . . 44

3.20 Tracking errors under control protocols $(3.27),(3.30)$ and $(3.31)$ with $c=60 \ldots \ldots$. . . . . 45

3.21 Phase trajectories of the leader agent and the follower agents under control protocols (3.27),

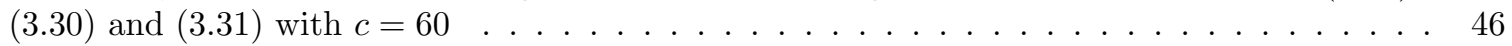

3.22 Strongly-connected directed graph: approximation of $f_{i}\left(x_{i}\right)$ (blue dashed-dotted lines) by RBFNNs (black dashed lines) for the 5 follower agents under control protocols (3.27), (3.30) and $(3.31)$ with $c=60 \ldots \ldots \ldots \ldots \ldots \ldots \ldots$

3.23 A switching strongly-connected directed graph communication topology with infinite many different graph . . . . . . . . . . . . . . . . . . . . . . . 4 49

3.24 Infinite topology set $G$ case: tracking errors under control protocols (3.27), (3.30) and (3.31)

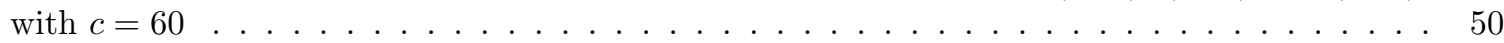

3.25 Infinite topology set $G$ case: phase trajectories of the leader agent and the follower agents under control protocols $(3.27),(3.30)$ and $(3.31)$ with $c=60 \ldots \ldots \ldots \ldots \ldots$

4.1 A switching directed graph interaction topology without a leader $\ldots \ldots \ldots \ldots$

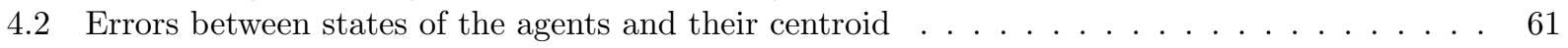

4.3 A switching directed graph interaction topology with a stationary leader . . . . . . . . . 63

4.4 Agents' state tracking errors with a stationary leader . . . . . . . . . . . . . . . . 64

5.1 The 1st dimension tracking errors between follower agents and the virtual leader under control

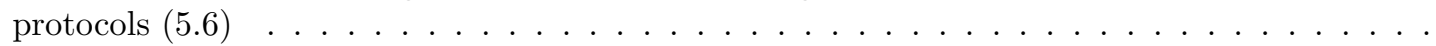


5.2 The 2nd dimension tracking errors between follower agents and the virtual leader under control protocols $(5.6) \ldots \ldots \ldots \ldots \ldots \ldots \ldots$

5.3 Tracking performance under control protocols (5.6) without the proposed topology connectivity maintenance mechanism . . . . . . . . . . . . . . . . . . . 91

5.4 Tracking performance under control protocols (5.6) with the proposed topology connectivity maintenance mechanism . . . . . . . . . . . . . . . . . . . . . . . 92

5.5 The 1st dimension tracking errors between follower agents and the virtual leader under control

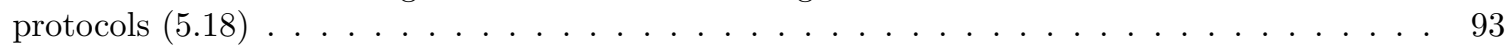

5.6 The 2nd dimension tracking errors between follower agents and the virtual leader under control

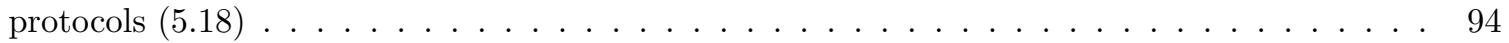




\section{Chapter 1}

\section{Introduction}

\subsection{Overview of Multi-agent Networks}

Distributed coordination of complex multi-agent networks has undergone rapid progress over the past decades. Due to the widely recognized advantages and potentials in various applications such as large scale sensor networks, robotic networks, distributed computing clouds, power grid, social networks and biological networks, the distributed coordination problems in complex multi-agent networks have received much attention from researchers in various disciplines including control, optimization, computer science, physics, social science and biology. Some earliest influential research works on the distributed multi-agent systems are perhaps the work in social science by DeGroot [1], the work in distributed decision making by Tsitsiklis ([2,3]), the work in statistical physics by Vicsek et al. [4], and the work in distributed computing by Lynch [5]. Within the control theory community, in the past two decades, much research effort (e.g., [6-14, 17-37,41,47-49]) has been spent on distributed consensus and synchronization control, which are two very important coordinated control problems in engineering multi-agent networks.

A multi-agent network consists of a number of interacting intelligent agents that cooperate to solve a problem or to achieve a common objective. Each intelligent agent in the system must be endowed with autonomy and social ability. That is, each agent must be able to observe the environment and other agents in the system and to react based on such observations. Intelligent agents can be any entities that possess such two properties, such as, unmanned vehicles, robots and biological objects.

The most significant feature and the biggest advantage of engineering multi-agent networks is perhaps that the power of the system as a whole can be greater than the sum of individuals' power. As a result, engineering multi-agent networks can be used to solve large-scale or complicated problems that are not 
solvable by individual systems.

Regardless of the control algorithms employed, coordinated control in engineering multi-agent networks relies heavily on interactions among agents and for this reason the interaction topology plays an essential role in the development of the distributed coordination control algorithms as well as the analysis of their performance. Two major approaches have been demonstrated to be effective in developing and analyzing control protocols for multi-agent networks with time varying interaction topologies. They are the common Lyapunov function approach and the multiple Lyapunov function approach [51].

\subsection{Distributed Consensus Control of Multi-agent Networks un- der Dynamic Interaction Topologies}

Consensus control is an important problem in coordinated control of multi-agent networks. Many algorithms used to solve other coordination problems, including formation control and swarm tracking, originate from consensus protocols. Consensus control is concerned with reaching a network-wide agreement on some quantities of interest while each agent can only access local neighborhood information. Applications of consensus control include cooperative estimation in wireless sensor networks and management of distributed database.

The consensus control problem of multi-agent networks under time varying interaction topologies has been intensively studied by researchers. To be specific, for multi-agent systems with first order integrator dynamics, Jadbabaie et al. [6] proposed distributed consensus control protocols such that the group of agents can reach consensus under jointly connected switching interaction topologies. The interaction graph considered in [6] is undirected, and all edges in the graph have identical weights. In 2005, Ren and Beard [8] extended the results of [6] to directed interaction graphs. They proposed distributed control protocols for multi-agent systems with first order integrator agent dynamics and showed that the information consensus can be achieved asymptotically under their control algorithms if the union of the switching directed interaction graphs contains a spanning tree frequently enough. Hong et. al [17] in 2007 proposed a Lyapunov-based approach to solving the second order consensus control problem for multi-agent systems with a stationary leader, which stands for a fixed objective point, under switching jointly connected interaction topologies. The interaction topology considered in [17] is undirected, and some additional assumptions about the interaction graphs which are more restrictive than those in [8] are made to derive the results. In 2012, Su and Huang [24] proposed consensus control protocols for a class of multi-agent systems with higher order agent dynamics under switching jointly connected undirected interaction topologies. With the assumption that the linear 
multi-agent system is marginally stable, they showed that consensus can be achieved by the group of agents under their proposed protocols. Both the leaderless and leader following consensus problems were addressed in their work. However, as the authors have pointed out, the tool they used in [24] is not applicable to directed graphs, and thus it is not obvious how their results could be extended to multi-agent systems with directed interaction topologies. It is pointed out here that, for distributed consensus control under a jointly connected directed interaction topology, the existing literature is limited to the simplest case of agent dynamics, namely, single integrator agent dynamics [8].

Here, the exact definitions about graph topology connectivity (e.g., joint connectivity) are referred to Section 2.1 ("Graph Theory") of this thesis.

\subsection{Distributed Synchronization Control of Multi-agent Networks under Dynamic Interaction Topologies}

Different from consensus, which is concerned with reaching a network-wide agreement on some quantities of interest, synchronization defines the correlated-in-time behavior between different agents achieved by local interaction strategies. Namely, distributed synchronization control aims at driving all agents to achieve the same states, and such synchronized states are allowed to be time varying. Synchronization control can also be employed to achieve coordinated tracking objectives in multi-agent networks.

Early works on distributed synchronization control of multi-agent networks mainly focus on the case of known linear agent dynamics. More recently, some emerging research started to address the distributed synchronization control of multi-agent networks with unknown nonlinearities. In particular, the consensus problem for multi-agent systems with unknown nonidentical nonlinear dynamics and disturbances, was first studied by Hou et al. [11] for a fixed connected undirected graph communication topology. In a pair of papers, Das and Lewis $([12,13])$ extended the work [11] to a fixed strongly-connected digraph communication topology, and they considered the synchronization problem with a leader. First-order systems were considered in [12], and second-order systems were considered in [13]. However, unfortunately, the results in ([12, 13]) do not hold due to a technical error. In addition, for such a class of multi-agent network with unknown nonidentical nonlinear agent dynamics, there is no existing literature addressing the distributed synchronization control problem under a time varying interaction topology. 


\subsection{Distributed Coordinated Tracking of Multi-agent Networks under State Dependent Dynamic Interaction Topologies}

In most of the literature on multi-agent systems with switching interaction topologies, the network interaction topology connectivity is simply defined as a function of time, and is not dependent on the agent states. However, in many real world applications, the network topology might actually depend on the agent states. For example, the connectivity might depend on the distance between agents if equipped sensors are used for the information exchange between agents.

To be specific, in the case that the interaction topology is defined based on physical proximity and all agents have identical sensing radius, one well-known work [9] is by Olfati-Saber, who proposed a distributed virtual leader tracking control protocol for multi-agent systems with second order integrator dynamics. In particular, under the assumptions that all agents are informed and that the virtual leader travels at a constant velocity, Olfati-Saber showed that, the proposed virtual leader tracking control algorithm, which incorporates a navigational feedback, enables all the follower agents to track a virtual leader. Su et al. [22] extended the results in [9] to the case that only a fraction of the agents are informed. They proposed a revised version of Olfati-Saber's algorithm and showed that, when only a fraction of agents are informed, their proposed control algorithm still enables all the informed agents to move with the desired velocity, and an uninformed agent to also move with the same desired velocity if it can be influenced by the informed agents from time to time during the evolution. It is emphasized here that the dynamic network interaction topology is not required to be connected at each time instant to establish the results. Note that, the proposed distributed control protocols in [9] and [22] involve quite complicated nonlinear functions, and topology connectivity maintenance was not considered. The previous works $([20,21])$ also extended [9] to the case that only a fraction of the follower agents are informed, and their proposed control protocols work for the case that the virtual leader has a time varying velocity. However, in both [20] and [21], in order to drive the follower agents to track the virtual leader, it is required that the undirected interaction topology is connected all the time, which is restrictive.

\subsection{Research Objectives}

A summary of some existing research and problems that remain unresolved is as follows.

- Distributed synchronization control problem for multi-agent networks with unknown nonlinearities has been studied in ([11-13]), but time varying interaction topologies were not considered. In addition, for 
agent dynamics, only first order and second order systems were addressed. Moreover, the results for fixed directed interaction topologies $([12,13])$ do not hold, due to a technical error.

- Distributed consensus control problem for multi-agent networks under a jointly connected directed interaction topology has been studied in [8], but only the simplest case of agent dynamics was considered, namely, single integrator agent dynamics.

- Distributed virtual leader tracking problem for multi-agent networks under a state dependent dynamic interaction topology has been studied in $([9,20-22])$. However, in ([20,21]), a restrictive assumption about the network interaction topology connectivity was made, namely, the topology is connected at each time instant. In $([9,22])$, the proposed controller structure is quite complex and involves complicated nonlinear functions. Moreover, the velocity information of the virtual leader is required in all these works $([9,20-22])$.

In view of the problems summarized in the above list, in this research we aimed to:

- Design distributed synchronization control protocols for multi-agent networks with unknown nonlinearities and with general $n$th order agent dynamics, under time varying interaction topologies. A common Lyapunov function method is employed to establish our results on time varying undirected interaction topologies, while a multiple Lyapunov function method is developed to establish our results on time varying directed interaction topologies.

- Design distributed consensus control protocols for high order linear multi-agent systems under a jointly connected directed interaction topology. The closed-loop performance is proved.

- Design distributed virtual leader tracking control protocols for multi-agent networks under a state dependent time varying interaction topology. Our proposed control protocols are much simpler than those in $([9,22])$, and we do not pose the restrictive connectivity assumption that the topology is connected all the time as in $([20,21])$. Then, we further extend the results to the case that when the velocity information of the virtual leader is not available to follower agents. Finally, we design a novel topology connectivity enhancing mechanism to help ensure virtual leader tracking in real world implementations. In comparison with the existing topology connectivity preserving algorithms, our proposed topology connectivity enhancing mechanism is effective in maintaining both the initially existent topology edges and the newly formed topology edges. 


\subsection{Dissertation Outline}

The remainder of this dissertation will be organized as follows. In Chapter 2, mathematical tools and concepts are introduced. Chapter 3 presents our solutions to the distributed synchronization control problems for multi-agent networks with unknown nonlinearities. In Chapter 4, we address the distributed consensus control problem for high order linear multi-agent systems under a jointly connected interaction topology. In Chapter 5 , we presents research results on the distributed virtual leader tracking for multi-agent networks under a state dependent time varying interaction topology. Finally, conclusions regarding this work are drawn in Chapter 6, where possible future works are discussed as well. This dissertation research has resulted in the publications listed in ([38-46]). 


\section{Chapter 2}

\section{Fundamentals}

\subsection{Graph Theory}

Distributed coordinated control of multi-agent systems, including synchronization, consensus and tracking, relies on the information exchange among the agents. In this work, the communication topology network among the agents is represented by a directed (or undirected) graph, which might change over time.

Definition $1 A$ directed weighted graph $G(t)=\{V, E(t), A(t)\}$ consists of a nonempty set of nodes $V=$ $\left\{v_{1}, v_{2}, \cdots, v_{N}\right\}$, a set of edges $E(t) \subset V \times V$, and an adjacency matrix $A(t)=\left[a_{i j}\right](t) \in R^{N \times N}$ which specify the weights of the edges. The ordered pair $\left(v_{i}, v_{j}\right) \in E(t)$ if and only if there exists a directional communication link from node $v_{i}$ to node $v_{j}$ at time $t$. For entries of the adjacency matrix $A(t), a_{i j}(t)>0$ if $\left(v_{j}, v_{i}\right) \in E(t)$, and $a_{i j}(t)=0$ otherwise. A directed edge $\left(v_{j}, v_{i}\right)$ at time $t$ denotes that agent $i$ has access to the information of agent $j$ at time $t$. Let $N_{i}(t)$ denote the index set of the neighboring nodes of $v_{i}$ at time $t$, that is, $N_{i}(t)=\left\{1 \leq j \leq N:\left(v_{j}, v_{i}\right) \in E(t)\right\}$.

Remark 1 In definition 1, for the special case that the edges are bidirectional and $a_{i j}(t) \equiv a_{j i}(t), G(t)=$ $\{V, E(t), A(t)\}$ is said to be an undirected graph.

Remark 2 In definition 1, if $E(t)$ and $A(t)$ do not change over time, then $G(t)$ is said to be a fixed graph, and the notation of the graph can be simplified as $G=\{V, E, A\}$.

Definition 2 In a fixed undirected graph, a path is defined as a sequence of nodes in which any two consecutive nodes are linked by an edge. A fixed undirected graph $G=\{V, E, A\}$ is said to be connected if there exists an undirected path between any pair of two nodes $v_{i}$ and $v_{j}$, where $v_{i} \in V, v_{j} \in V, i \neq j$. 
Definition 3 In a fixed directed graph, a directed path of length $l$ is a sequence of directed edges $\left(v_{i_{1}}, v_{i_{2}}\right),\left(v_{i_{2}}, v_{i_{3}}\right), \cdots,\left(v_{i_{l}}, v_{i_{(l+1)}}\right)$. A fixed directed graph $G=\{V, E, A\}$ is said to be strongly connected if there exists a directed path from any node $v_{i}$ to any node $v_{j}$, where $v_{i} \in V, v_{j} \in V, i \neq j$.

Definition 4 A time varying undirected weighted graph $G(t)=\{V, E(t), A(t)\}$ is said to be jointly connected if, there exists a positive constant $T>0$, such that, for any $t \geq 0$, the union of the graphs over the time interval $[t, t+T)$ is connected.

Definition 5 A time varying directed weighted graph $G(t)=\{V, E(t), A(t)\}$ is said to be jointly connected if, there exists a positive constant $T>0$, such that, for any $t \geq 0$, the union of the graphs over the time interval $[t, t+T)$ contains a directed spanning tree.

Definition 6 Define the Laplacian matrix of graph $G(t)=\{V, E(t), A(t)\}$ as $L(t)=D(t)-A(t)$, where $D(t)=\operatorname{diag}\left\{d_{1}, d_{2}, \cdots, d_{N}\right\}$ with $d_{i}=\sum_{j=1}^{N} a_{i j}(t), i=1,2, \cdots, N$. For an undirected graph, $L(t)=(L(t))^{T}$.

Definition 7 For a multi-agent network with $N$ follower agents and one leader agent, define a diagonal matrix $B=\operatorname{diag}\left\{b_{i}\right\} \in R^{N \times N}$, where $b_{i}>0$ if follower agent $i$ has access to the information of the leader agent, and $b_{i}=0$ otherwise.

\subsection{Switched System Theory}

Switched system theory is very useful in developing and analyzing distributed control protocols for multi-agent networks under a switching communication topology. In particular, the common Lyapunov function approach and the multiple Lyapunov function approach are two major approaches that have been demonstrated to be effective in analyzing the performance of such a multi-agent network with time varying communication topologies [51].

Lyapunov function approach is a fundamental tool in control theory to analyze stability of linear and nonlinear systems, including multi-agent network systems. For multi-agent network with a time varying interaction topology, most existing works rely on the construction of a common Lyapunov function. However, in many situations, a common Lyapunov function might be very difficult to know or does not exist at all. In such cases, the multiple Lyapunov function approach to solving the stability analysis problems of multi-agent systems with switching communication topologies is a promising avenue to pursue. To be specific, in such a case, a series of different Lyapunov functions would be constructed, where different Lyapunov functions correspond to different communication interaction topologies. Then these constructed Lyapunov functions would be used together to analyze the performance of the multi-agent networks under a time 

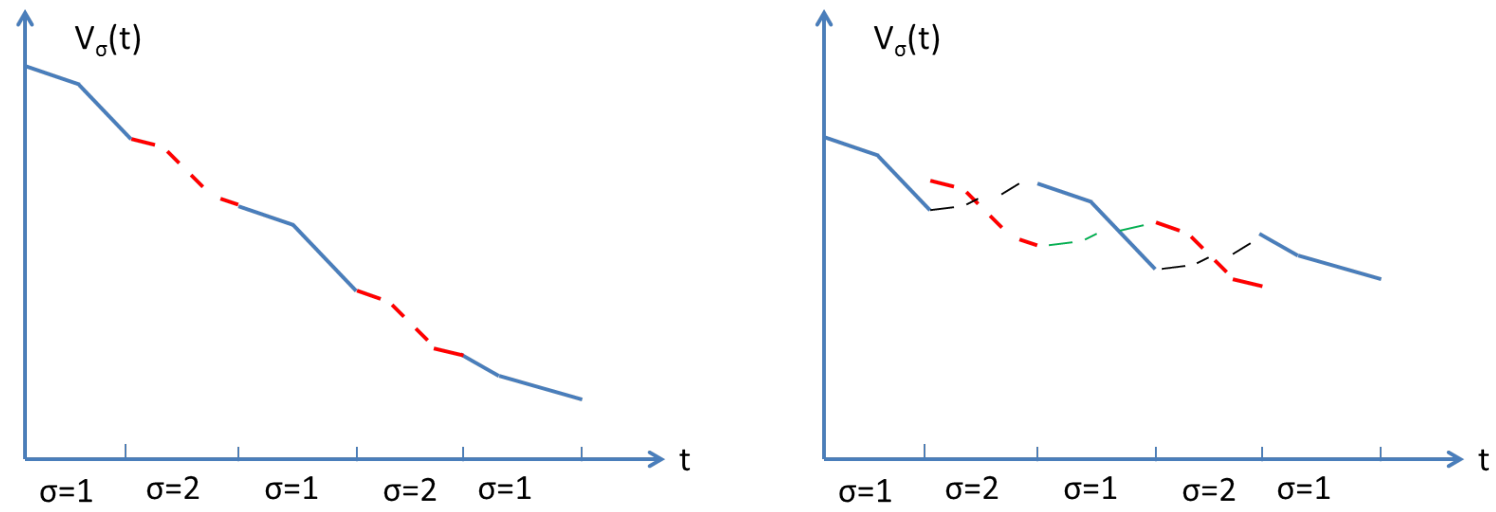

Figure 2.1: Two Lyapunov functions (blue solid thick lines correspond to active $V_{1}$, red dashed thick lines correspond to active $V_{2}$, black dashed thin lines correspond to inactive $V_{1}$, green dashed thin lines correspond to inactive $V_{2}$ ): a) left figure - continuous $V_{\sigma}$; b) right figure - discontinuous $V_{\sigma}$

varying interaction topology. It is pointed out here that, to use the method of multiple Lyapunov function approach to prove system stability, it is required that the total number of the constructed different Lyapunov functions is finite.

To illustrate the basic idea of multiple Lyapunov function approach, consider the simple case of a switched system that consists of only two individual subsystems $S y s_{1}$ and $S y s_{2}$, both of which are (globally) asymptotically stable. Let $V_{1}$ and $V_{2}$ be their respective (radially unbounded) Lyapunov functions. We are interested in the situation where a common Lyapunov function for the two systems is not known or does not exist. Let the system switch between $S y s_{1}$ and $S y s_{2}$ in a sequence defined by a switching signal $\sigma(t)$, whose value set is $\{1,2\}$. Namely, if $\sigma(t)=1$, the subsystem $S y s_{1}$ is active at time $t$, and if $\sigma(t)=2$, the subsystem $S y s_{2}$ is active at time $t$. Let $t_{i}, i=1,2, \cdots$, be the switching time. In the absence of a common Lyapunov function, the stability of such a switched system in general depends on the switching signal $\sigma(t)$. If it so happens that the values of $V_{1}$ and $V_{2}$ coincide at each switching time, i.e., $V_{\sigma\left(t_{i-1}\right)}\left(t_{i}\right)=V_{\sigma\left(t_{i}\right)}\left(t_{i}\right)$ for all $i$, then $V_{\sigma}$ is a continuous Lyapunov function for the switched system, and asymptotic stability follows. This situation is depicted in Fig 2.1(a). In general, however, the function $V_{\sigma}$ will be discontinuous. While each $V_{p}$, $p \in\{1,2\}$, decreases when the $p$ th subsystem is active, it may increase when the $p$ th subsystem is inactive. This behavior is shown in Fig 2.1(b). The basic idea that allows one to show asymptotic stability in this case is as follows. Let us look at the values of $V_{p}$ at the beginning of each time interval on which $\sigma=p$. For the switched system to be asymptotically stable, these values must form a decreasing sequence for each $p$. 


\section{Chapter 3}

\section{Distributed Synchronization Control of Multi- agent Networks With Unknown Nonlinearities under Connected Dynamic Interaction Topolo- gies}

\subsection{Problem Statement}

We consider a network of $N$ agents. The dynamics of each agent $i, i=1,2, \cdots, N$, in the network are described as

$$
\left\{\begin{aligned}
\dot{x}_{i 1} & =x_{i 2} \\
\dot{x}_{i 2} & =x_{i 3} \\
& \vdots \\
\dot{x}_{i n} & =f_{i}\left(x_{i 1}, x_{i 2}, \cdots, x_{i n}\right)+u_{i}+w_{i}
\end{aligned}\right.
$$

where $f_{i}\left(x_{i 1}, x_{i 2}, \cdots, x_{i n}\right)$ is an unknown nonlinear function, $u_{i}$ is the control input, and $w_{i}$ is the disturbance. On the other hand, the dynamics of the leader agent are described as follows,

$$
\left\{\begin{aligned}
\dot{x}_{01} & =x_{02} \\
\dot{x}_{02} & =x_{03} \\
& \vdots \\
\dot{x}_{0 n} & =f_{0}\left(x_{01}, x_{02}, \cdots, x_{0 n}, t\right)
\end{aligned}\right.
$$


where $f_{0}\left(x_{01}, x_{02}, \cdots, x_{0 n}, t\right)$ is an unknown nonlinear function.

As with the literature on this type of problems, we make the following standard assumptions on the agents' dynamics.

Assumption 1 The dynamics of the agents satisfy the following assumptions:

\section{A1. The disturbance $w_{i}$ is bounded for all $i$;}

A2. The function $f_{i}\left(x_{i 1}, x_{i 2}, \cdots, x_{i n}\right)$ is smooth for all $i$;

A3. The neural network activation functions $\varphi_{i}\left(x_{i}\right)$ are bounded for all $i$;

A4. The function $f_{0}\left(x_{01}, x_{02}, \cdots, x_{0 n}, t\right)$ is smooth;

A5. The target trajectory $\left(x_{01}(t), x_{02}(t), \cdots, x_{0 n}(t)\right)$ is bounded.

Neural network is usually employed to approximate unknown functions. It has been proved [52] that any continuous function $f(x)$ can be approximated by the radial basis function neural network (RBFNN) with one hidden layer on a compact set to an arbitrary accuracy. That is, for a continuous function $f(x)$ and any given positive constant $\varepsilon_{0}$, there exists a number $\eta_{0}$ such that, for any network of $\eta \geq \eta_{0}$ neurons, there exists an ideal neural network weight matrix $W$ such that the function approximation error $\varepsilon=\left\|f(x)-W^{\mathrm{T}} \varphi(x)\right\| \leq \varepsilon_{0}$, where $\varphi(x)$ are the radial basis functions of the neural network. On the other hand, for a neural network with a given number of neurons $\eta_{0}$, there exists a positive constant $\varepsilon_{0}$ and an ideal neural network weight matrix $W$ such that the function approximation error $\varepsilon=\left\|f(x)-W^{\mathrm{T}} \varphi(x)\right\| \leq \varepsilon_{0}$. In this Chapter, we will use RBFNN to approximate the smooth unknown nonlinearities $f_{i}\left(x_{i 1}, x_{i 2}, \cdots, x_{i n}\right)$.

Our objective in this chapter is to design distributed control protocols for synchronization of all agents to the leader agent, referred to as the cooperative tracker problem. Namely, we would like to design $u_{i}$ using only local neighborhood information of agent $i$ such that $x_{i j}(t)-x_{0 j}(t) \rightarrow 0$ as $t \rightarrow \infty$, for all $i=1,2, \cdots, N$, $j=1,2, \cdots, n$. Agents in the multi-agent system have $n$th order system dynamics. Different kinds of communication topologies would be addressed. In particular, in Section 3.2, we consider the case of a fixed strongly-connected directed graph communication topology. In Section 3.3, we presents results on switching connected undirected graph communication topologies. In Section 3.4, we presents results on switching connected directed graph communication topologies.

The following fact is important for the control design and analysis in this chapter.

Lemma 1 [53] Suppose $x(t)$ is a smooth function of $t$, and assume that $x(0), \dot{x}(0), \cdots, x^{n-1}(0)$ are all bounded. Define $s(x, t)=\left(\lambda+\frac{d}{d t}\right)^{n-1} x(t)$, where $\lambda>0$ is a constant. If $s(x, t)$ is bounded for all $t$, then $x(t)$, 
$\dot{x}(t), \cdots, x^{n-1}(t)$ will be bounded for all $t$. Besides, if $s(x, t) \rightarrow 0$ as $t \rightarrow \infty$, then $x(t) \rightarrow 0, \dot{x}(t) \rightarrow 0, \cdots$, $x^{n-1}(t) \rightarrow 0$ as $t \rightarrow \infty$.

\subsection{Synchronization Control of $n$ th-order Systems with a Fixed Strongly-connected Directed Graph Communication Topology}

We consider a network of $N$ agents. Each agent is a $n$ th-order system whose dynamics are described by (3.1). Besides the $N$ agents, there is also a leader agent, whose dynamics are described by (3.2).

The network communication topology is represented by a directed graph $G_{N}=(V, E)$, where $V=$ $\left\{v_{1}, v_{2}, \cdots, v_{N}\right\}$ is a finite, nonempty set of $N$ vertices (each vertex denotes an agent in the network), and $E \in V \times V$ is a set of edges of the graph. An edge $\left(v_{i}, v_{j}\right)$ denotes that agent $j$ has access to the information of agent $i$. A directed path of length $l$ is a sequence of edges $\left(v_{i_{1}}, v_{i_{2}}\right),\left(v_{i_{2}}, v_{i_{3}}\right), \cdots,\left(v_{i_{l}}, v_{i_{(l+1)}}\right)$. Agent $i$ is said to be in the neighborhood of agent $j$ if $\left(v_{i}, v_{j}\right) \in E$. Each agent in the network has only limited communication capability and can only access the information in its neighborhoods. Define the adjacency matrix as $A=\left[a_{i j}\right]$ with $a_{i j}>0$ if $\left(v_{j}, v_{i}\right) \in E$, and $a_{i j}=0$ otherwise. The weighted in-degree matrix $D$ is

defined as a diagonal matrix $D=\operatorname{diag}\left\{d_{i}\right\}$ with $d_{i}=\sum_{j=1}^{N} a_{i j}$. The graph Laplacian matrix is defined as $L=D-A$. Besides the $N$ agents in $V$, the leader agent is labeled as $v_{0}$. An edge from the leader agent $v_{0}$ to agent $i$ denotes that agent $i$ has access to the information of the leader agent, with the communication weight defined as $b_{i}>0$. Otherwise, $b_{i}=0$. Define matrix $B=\operatorname{diag}\left\{b_{i}\right\}$.

Following fact is important for control design and analysis in this section. M-matrix is defined as a square matrix with all off-diagonal elements non-positive and all principal minors non-negative [14].

Lemma 2 [14] If a directed graph $G_{N}$ is strongly connected and $B=\operatorname{diag}\left\{b_{i}\right\}$ has at least one $b_{i}>0$, then $L+B$ is a non-singular M-matrix. It follows that $(L+B)^{T}$ is a non-singular M-matrix. Define $s=\left[s_{1} s_{2} \cdots s_{N}\right]^{T}=\left((L+B)^{T}\right)^{-1} \underline{1}$, where $\underline{1}$ denotes the vector $\left[\begin{array}{lll}1 & \cdots & 1\end{array}\right]^{T}$. Define matrix $S=\operatorname{diag}\left\{s_{i}\right\}$. Then $S>0$ and $Q=(L+B)^{T} S+S\left((L+B)^{T}\right)^{T}=(L+B)^{T} S+S(L+B)>0$.

In the remainder of this section, Section 3.2.1 provides the control algorithm description and main results, and section 3.2.2 presents the proof of the main results.

\subsubsection{Control Protocols and the Closed-loop System Performance}

In order to synchronize the states of agents in the network to the state of the leader agent, we propose following control law,

$$
u_{i}=c r_{i}-\hat{f}_{i}\left(x_{i}\right)=c r_{i}-\hat{W}_{i}^{\mathrm{T}} \varphi_{i}\left(x_{i}\right)
$$


where

$$
e_{i k}=\sum_{j \in N_{i}} a_{i j}\left(x_{j k}-x_{i k}\right)+b_{i}\left(x_{0 k}-x_{i k}\right)
$$

and

$$
r_{i}=\left(1+\frac{d}{d t}\right)^{n-1} e_{i 1}=C_{n-1}^{0} e_{i 1}+C_{n-1}^{1} e_{i 2}+\cdots+C_{n-1}^{n-1} e_{i n}
$$

Also, $a_{i j}$ and $b_{i}$ are defined in Section 3.2, $c>0$ is a control gain parameter, $\varphi_{i}\left(x_{i}\right)$ are the activation functions of the neural network, and $\hat{W}_{i}$ are the neural network weights, which are tuned according to

$$
\dot{\hat{W}}_{i}= \begin{cases}-\chi_{i} \varphi_{i} r_{i}^{\mathrm{T}}+\chi_{i} \frac{r_{i}^{\mathrm{T}} \hat{W}_{i}^{\mathrm{T}} \varphi_{i}}{\operatorname{Tr}\left\{\hat{W}_{i}^{\mathrm{T}} \hat{W}_{i}\right\}} \hat{W}_{i}, & \text { if }-r_{i}^{\mathrm{T}} \hat{W}_{i}^{\mathrm{T}} \varphi_{i}>0 \text { and } \\ -\chi_{i} \varphi_{i} r_{i}^{\mathrm{T}}, & \operatorname{Tr}\left\{\hat{W}_{i}^{\mathrm{T}} \hat{W}_{i}\right\}=W_{\max i}, \\ & \text { otherwise }\end{cases}
$$

where $W_{\max i}$ is a given positive constant for limiting the neural network weights, and $\chi_{i}>0$ is a given constant which controls the adaption rate of $\hat{W}_{i}$. This neural network weight matrix updating law is adapted from [11]. The following lemma, established in [11], guarantees the boundedness of $\widehat{W}_{i}$.

Lemma 3 [11] Consider the neural network weight matrix updating law (3.6). If the initial value of the neural network weight matrix satisfies

$$
\operatorname{Tr}\left(\widehat{W}_{i}^{T}(0) \widehat{W}_{i}(0)\right) \leq W_{\max i}
$$

then,

$$
\operatorname{Tr}\left(\widehat{W}_{i}^{T}(t) \widehat{W}_{i}(t)\right) \leq W_{\max i}, t \geq 0, i=1,2, \cdots, N
$$

Define the synchronization error as

$$
\delta_{i k}=x_{i k}-x_{0 k}, i=1,2, \cdots, N, k=1,2, \cdots, n .
$$

Denote $\delta_{1}=\left[\begin{array}{llll}\delta_{11} & \delta_{21} & \cdots & \delta_{N 1}\end{array}\right]^{\mathrm{T}}, \delta_{2}=\left[\begin{array}{llll}\delta_{12} & \delta_{22} & \cdots & \delta_{N 2}\end{array}\right]^{\mathrm{T}}, \delta_{n}=\left[\begin{array}{llll}\delta_{1 n} & \delta_{2 n} & \cdots & \delta_{N n}\end{array}\right]^{\mathrm{T}}$ and $\delta=\left[\begin{array}{llll}\delta_{1}^{\mathrm{T}} & \delta_{2}^{\mathrm{T}} & \cdots & \delta_{n}^{\mathrm{T}}\end{array}\right]^{\mathrm{T}}$. Then, our main results on the synchronization of the states of all agents to the state of the leader agent can be stated in the following theorem 1, whose proof is presented in Section 3.2.2.

Theorem 1 Consider a system of $N$ agents whose dynamics are described by (3.1) and steered by the control protocol specified by (3.3), (3.6) and (3.7). Consider that the leader agent's dynamics are described by (3.2). Suppose that Assumption 1 is satisfied. Assume that the digraph communication topology is fixed and strongly 
connected, and the leader agent is at least connected to one agent in the network. Then, given any bounded set $\mathcal{X}_{0}$, for any initial synchronization error $\delta(0)$ that belongs to $\mathcal{X}_{0}$, there exists a positive constant $c_{\mathcal{X}_{0}}$ such that the synchronization error $\delta$ is bounded as long as $c \geq c_{\mathcal{X}_{0}}$. Furthermore, the ultimate bound on the synchronization error $\delta$ can be reduced arbitrarily by increasing the value of the control parameter $c$.

\subsubsection{Theoretical Analysis}

To prove Theorem 1, we choose the Lyapunov function

$$
V=\frac{1}{2} r^{\mathrm{T}} S r
$$

where $r=\left[r_{1} r_{2} \cdots r_{N}\right]^{\mathrm{T}}$ and $S=\operatorname{diag}\left\{s_{i}\right\}$, with $s_{i}$ being the parameters associated with the graph as defined in Lemma 2.

Denote $e_{1}=\left[\begin{array}{llll}e_{11} & e_{21} & \cdots & e_{N 1}\end{array}\right]^{\mathrm{T}}, e_{2}=\left[\begin{array}{llll}e_{12} & e_{22} & \cdots & e_{N 2}\end{array}\right]^{\mathrm{T}}, e_{n}=\left[\begin{array}{llll}e_{1 n} & e_{2 n} & \cdots & e_{N n}\end{array}\right]^{\mathrm{T}}$ and $e=\left[\begin{array}{llll}e_{1}^{\mathrm{T}} & e_{2}^{\mathrm{T}} & \cdots & e_{n}^{\mathrm{T}}\end{array}\right]^{\mathrm{T}}$.

In view of $(3.4),(3.5),(3.9)$, we have

$$
\begin{aligned}
e_{1} & =-(L+B) \delta_{1}, \\
e_{2} & =-(L+B) \delta_{2}, \\
& \vdots \\
e_{n} & =-(L+B) \delta_{n}, \\
r & =C_{n-1}^{0} e_{1}+C_{n-1}^{1} e_{2}+\cdots+C_{n-1}^{n-1} e_{n} .
\end{aligned}
$$

Since the communication topology is strongly connected, $L+B$ is nonsingular, and hence

$$
\begin{aligned}
\left\|e_{1}\right\| & \leq \bar{\sigma}(L+B)\left\|\delta_{1}\right\|, \\
\left\|e_{2}\right\| & \leq \bar{\sigma}(L+B)\left\|\delta_{2}\right\|, \\
& \vdots \\
\left\|e_{n}\right\| & \leq \bar{\sigma}(L+B)\left\|\delta_{n}\right\|,
\end{aligned}
$$

and

$$
\begin{aligned}
& \left\|\delta_{1}\right\| \leq \frac{\left\|e_{1}\right\|}{\underline{\sigma}(L+B)}, \\
& \left\|\delta_{2}\right\| \leq \frac{\left\|e_{2}\right\|}{\underline{\sigma}(L+B)},
\end{aligned}
$$




$$
\left\|\delta_{n}\right\| \leq \frac{\left\|e_{n}\right\|}{\underline{\sigma}(L+B)}
$$

Here and later in this proof, for a nonsingular matrix $M, \bar{\sigma}(M)$ and $\underline{\sigma}(M)$ denote the maximal and minimal singular values of $M$, respectively.

Consequently,

$$
\|r\| \leq C_{n-1}^{0} \bar{\sigma}(L+B)\left\|\delta_{1}\right\|+C_{n-1}^{1} \bar{\sigma}(L+B)\left\|\delta_{2}\right\|+\cdots+C_{n-1}^{n-1} \bar{\sigma}(L+B)\left\|\delta_{n}\right\|
$$

Let $h_{0}>0$ be such that $\mathcal{X}_{0} \subseteq D_{0}=\left\{\delta:\left\|\delta_{1}\right\| \leq h_{0},\left\|\delta_{2}\right\| \leq h_{0}, \cdots,\left\|\delta_{n}\right\| \leq h_{0}\right\}$. We construct a level set $\Omega=\left\{e: V \leq \frac{1}{2} \bar{\sigma}(S) h^{2}\right\}$, where $h=2^{n-1} \bar{\sigma}(L+B) h_{0}$. It follows that $\mathcal{X}_{0} \subseteq \Omega$.

We now evaluate the derivative of $V$ within the level set $\Omega$ as follows,

$$
\dot{V}=r^{\mathrm{T}} S \dot{r}
$$

where

$$
\begin{aligned}
\dot{r} & =\frac{d}{d t}\left\{C_{n-1}^{0} e_{1}+C_{n-1}^{1} e_{2}+\cdots+C_{n-1}^{n-1} e_{n}\right\} \\
& =-(L+B)\left(f(x)-\underline{f}\left(x_{0}, t\right)+u+w\right)+C_{n-1}^{0} e_{2}+C_{n-1}^{1} e_{3}+\cdots+C_{n-1}^{n-2} e_{n} \\
& =-(L+B)\left(W^{\mathrm{T}} \varphi(x)+\varepsilon-\underline{f}\left(x_{0}, t\right)+c r-\hat{W}^{\mathrm{T}} \varphi(x)+w\right)+C_{n-1}^{0} e_{2}+C_{n-1}^{1} e_{3}+\cdots+C_{n-1}^{n-2} e_{n} \\
& =-(L+B)\left(\tilde{W}^{\mathrm{T}} \varphi(x)+\varepsilon-\underline{f}\left(x_{0}, t\right)+c r+w\right)+C_{n-1}^{0} e_{2}+C_{n-1}^{1} e_{3}+\cdots+C_{n-1}^{n-2} e_{n} .
\end{aligned}
$$

Here, $\varepsilon \leq \varepsilon_{0}$ is the ideal RBFNN function approximation error of $f(x)$ as defined in Section 3.1, $\varphi(x)=$ $\left[\varphi_{1}\left(x_{1}\right) \varphi_{2}\left(x_{2}\right) \cdots \varphi_{N}\left(x_{N}\right)\right]^{\mathrm{T}}, \hat{f}(x)=\left[\begin{array}{lll}\hat{f}_{1}\left(x_{1}\right) & \hat{f}_{2}\left(x_{2}\right) \cdots & \hat{f}_{N}\left(x_{N}\right)\end{array}\right]^{\mathrm{T}}, \underline{f}\left(x_{0}, t\right)=f_{0}\left(x_{0}, t\right)\left[\begin{array}{llll}1 & 1 & \cdots & 1\end{array}\right]^{\mathrm{T}}$ and $\tilde{W}=\tilde{W}=W-\hat{W}$ is the neural network weight approximation error with $W=\operatorname{diag}\left\{W_{i}\right\}$ being the ideal neural network weights and $\hat{W}=\operatorname{diag}\left\{\hat{W}_{i}\right\}$.

Therefore, we have

$$
\begin{aligned}
\dot{V} & =r^{\mathrm{T}} S \dot{r} \\
& =-r^{\mathrm{T}} S(L+B)\left(\tilde{W}^{\mathrm{T}} \varphi(x)+\varepsilon-\underline{f}\left(x_{0}, t\right)+w\right)+r^{\mathrm{T}} S\left(C_{n-1}^{0} e_{2}+C_{n-1}^{1} e_{3}+\cdots+C_{n-1}^{n-2} e_{n}\right) \\
& =-c r^{\mathrm{T}} S(L+B) r-r^{\mathrm{T}} S(L+B)\left(\varepsilon-\underline{f}\left(x_{0}, t\right)+w\right)-r^{\mathrm{T}} S(L+B) \tilde{W}^{\mathrm{T}} \varphi(x)+r^{\mathrm{T}} S\left(C_{n-1}^{0} e_{2}+C_{n-1}^{1} e_{3}+\cdots+C_{n-1}^{n-2} e_{n}\right)
\end{aligned}
$$


Since the communication topology is strongly connected, we know from Lemma 2 that the matrix $Q=S(L+B)+(L+B)^{\mathrm{T}} S$ is positive definite. Therefore, we have

$$
\begin{aligned}
\dot{V}= & -\frac{1}{2} c r^{\mathrm{T}} Q r-r^{\mathrm{T}} S(L+B)\left(\varepsilon-\underline{f}\left(x_{0}, t\right)+w\right)-r^{\mathrm{T}} S(L+B) \tilde{W}^{\mathrm{T}} \varphi(x)+r^{\mathrm{T}} S\left(C_{n-1}^{0} e_{2}+C_{n-1}^{1} e_{3}+\cdots+C_{n-1}^{n-2} e_{n}\right) \\
\leq & \frac{1}{2} c \underline{\sigma}(Q)\|r\|^{2}+\|r\| \bar{\sigma}(S) \bar{\sigma}(L+B)\left(\|\varepsilon\|+\left\|\underline{f}\left(x_{0}, t\right)\right\|+\|w\|+\|\tilde{W}\|\|\varphi(x)\|\right)+\|r\| \bar{\sigma}(S)\left(C_{n-1}^{0}\left\|e_{2}\right\|\right. \\
& \left.+C_{n-1}^{1}\left\|e_{3}\right\|+\cdots+C_{n-1}^{n-2}\left\|e_{n}\right\|\right)
\end{aligned}
$$

Note that the designed neural network weights tuning law ensures that $\operatorname{Tr}\left\{\hat{W}_{i}^{\mathrm{T}} \hat{W}_{i}\right\}$ would be bounded for all $i$. This fact was established as Lemma 2 in [11]. Then it follows that $\tilde{W}_{i}$ is bounded for all $i$. Consequently, we have

$$
\dot{V} \leq-\frac{1}{2} c \underline{\sigma}(Q)\|r\|^{2}+\|r\| B_{m}
$$

where $B_{m}=\bar{\sigma}(S) \bar{\sigma}(L+B)\left(\varepsilon_{0}+F_{0}+L_{0} \Phi_{m}+\Delta_{0}\right)+\left(2^{n-1}-1\right) \bar{\sigma}(S) M_{0}$ is a positive constant. Here $\varepsilon_{0}, F_{0}$, $L_{0}, \Phi_{m}$, and $\Delta_{0}$ respectively denote the bounds of $\varepsilon, \underline{f}\left(x_{0}, t\right), \tilde{W}, \varphi(x)$ and $w . M_{0}$ denotes an upper bound for all $\left\|e_{i}\right\|, i=2,3, \cdots, n$.

Let

$$
c_{\mathcal{X}_{0}}=\frac{2 B_{m}}{\underline{\sigma}(Q) h} .
$$

Then, for any $c \geq c_{\mathcal{X}_{0}}$, we have $\dot{V}<0$ as long as $\|r\|>h$. This implies that $\dot{V}<0$ as long as $V>\frac{1}{2} \bar{\sigma}(S) h^{2}$. Thus, the level set $\Omega$ is an invariant set for any $c \geq c_{\mathcal{X}_{0}}$.

We have now proven that $\|r(t)\|$ is bounded as long as $c \geq c_{\mathcal{X}_{0}}$. Recall that $r=\left(1+\frac{d}{d t}\right)^{n-1} e_{1}=$ $C_{n-1}^{0} e_{1}+C_{n-1}^{1} e_{2}+\cdots+C_{n-1}^{n-1} e_{n}$. It follows from lemma 1 that $\left\|e_{1}(t)\right\|,\left\|e_{2}(t)\right\|, \cdots,\left\|e_{n}(t)\right\|$ are all bounded since $\left\|e_{1}(0)\right\|,\left\|e_{2}(0)\right\|, \cdots,\left\|e_{n}(0)\right\|$ are bounded. In view of (3.13), the synchronization error $\delta$ is also bounded. Furthermore, it is easy to see that the ultimate bound of the synchronization error $\delta$ could be reduced arbitrarily by increasing the value of the control parameter $c$.

\subsection{Synchronization Control of $n$ th-order Systems with Switching Connected Undirected Graph Communication Topologies}

We consider a network of $N$ agents. Each agent is a $n$ th-order system whose dynamics are described by (3.1). Besides the $N$ agents, there is also a leader agent, whose dynamics are described by (3.2). 
The network communication topology is represented by a switching undirected weighted graph $G_{N}=$ $(V, E(t), A(t))$, where $V=\left\{v_{1}, v_{2}, \cdots, v_{N}\right\}$ is a finite, nonempty set of $N$ vertices (each vertex denotes an agent in the network), $E(t) \in V \times V$ is a set of edges of the graph which are dynamically changing in time, and $A=\left[a_{i j}(t)\right]$ with $a_{i j}(t) \geq 0$ and $a_{i j}(t)=a_{j i}(t)$ is the weighted adjacency matrix. At any time instant $t=t_{0}$, an edge $\left(v_{i}, v_{j}\right)$ denotes that agent $i$ can exchange information with agent $j$ at $t=t_{0}$, and $\left(v_{i}, v_{j}\right) \in$ $E(t) \Leftrightarrow\left(v_{j}, v_{i}\right) \in E(t)$. The adjacency matrix $A=\left[a_{i j}(t)\right]$ satisfies that $a_{i j}(t)>0$ if $\left(v_{j}, v_{i}\right) \in E(t)$, and $a_{i j}(t)=0$ otherwise. A undirected path of length $l$ is a sequence of edges $\left(v_{i_{1}}, v_{i_{2}}\right),\left(v_{i_{2}}, v_{i_{3}}\right), \cdots,\left(v_{i_{l}}, v_{i_{(l+1)}}\right)$. An undirected graph is said to be connected at $t=t_{0}$ if there exists a path between each pair of vertices $\left(v_{i}, v_{j}\right), i=1,2, \cdots, N, j=1,2, \cdots, N$ at $t=t_{0}$. Besides the $N$ agents in $V$, the leader agent is labeled as $v_{0}$. Again, at any time instant $t=t_{0}$, an edge between the leader agent $v_{0}$ and agent $i$ denotes that agent $i$ has access to the information of the leader agent at $t=t_{0}$, with the communication weight defined as $b_{i}(t)>0$. Otherwise, $b_{i}(t)=0$. Define matrix $B=\operatorname{diag}\left\{b_{i}(t)\right\}$. Define the weighted in-degree matrix $D$ as a diagonal matrix $D=\operatorname{diag}\left\{d_{i}(t)\right\}$ with $d_{i}(t)=\sum_{j=1}^{N} a_{i j}(t)$. Define the graph Laplacian matrix $L=D-A$.

In this section, the communication topology considered is a switching connected undirected weighted graph. Since the graph $G_{N}=(V, E(t), A(t))$ is connected all the time, it follows that $L+B$ are nonsingular all the time. Following assumption is needed in the control design and analysis in this section.

Assumption 2 Assume that the minimal singular value of $L+B$ is lower bounded by some positive constant $\sigma_{l}>0$, where $L$ and $B$ are defined above in Section 3.3.

In the remainder of this section, Section 3.3.1 provides the control algorithm description and main results, and Section 3.3.2 presents the proof of the main results.

\subsubsection{Control Protocols and the Closed-loop System Performance}

We propose the following control law,

$$
\begin{aligned}
u_{i} & =c r_{i}-\hat{f}_{i}\left(x_{i}\right) \\
& =c r_{i}-\hat{W}_{i}^{\mathrm{T}} \varphi_{i}\left(x_{i}\right),
\end{aligned}
$$

where

$$
e_{i k}=\sum_{j \in N_{i}} a_{i j}(t)\left(x_{j k}-x_{i k}\right)+b_{i}(t)\left(x_{0 k}-x_{i k}\right), k=1,2, \cdots, n,
$$

and

$$
r_{i}=\left(1+\frac{d}{d t}\right)^{n-1} e_{i 1}=C_{n-1}^{0} e_{i 1}+C_{n-1}^{1} e_{i 2}+\cdots+C_{n-1}^{n-1} e_{i n}
$$


Also, $a_{i j}(t)$ and $b_{i}(t)$ are defined above as in Section 3.3, $c>0$ is a control gain parameter, $\varphi_{i}\left(x_{i}\right)$ are the activation functions of the neural network, and $\hat{W}_{i}$ are the neural network weights, which are tuned according to

$$
\dot{\hat{W}}_{i}= \begin{cases}-\chi_{i} \varphi_{i} r_{i}^{\mathrm{T}}+\chi_{i} \frac{r_{i}^{\mathrm{T}} \hat{W}_{i}^{\mathrm{T}} \varphi_{i}}{\operatorname{Tr}\left\{\hat{W}_{i}^{\mathrm{T}} \hat{W}_{i}\right\}} \hat{W}_{i}, & \text { if }-r_{i}^{\mathrm{T}} \hat{W}_{i}^{\mathrm{T}} \varphi_{i}>0 \text { and } \\ -\chi_{i} \varphi_{i} r_{i}^{\mathrm{T}}, & \operatorname{Tr}\left\{\hat{W}_{i}^{\mathrm{T}} \hat{W}_{i}\right\}=W_{\max i}, \\ & \text { otherwise }\end{cases}
$$

with the initial value of the neural network weight matrix being chosen to satisfy

$$
\operatorname{Tr}\left(\widehat{W}_{i}^{\mathrm{T}}(0) \widehat{W}_{i}(0)\right) \leq W_{\max i}
$$

Here, $W_{\max i}$ is a given positive constant for limiting the neural network weights, and $\chi_{i}>0$ is a given constant which controls the adaption rate of $\hat{W}_{i}$.

Define the synchronization error as

$$
\delta_{i k}=x_{i k}-x_{0 k}, i=1,2, \cdots, N, k=1,2, \cdots, n .
$$

Denote $\delta_{1}=\left[\begin{array}{llll}\delta_{11} & \delta_{21} & \cdots & \delta_{N 1}\end{array}\right]^{\mathrm{T}}, \delta_{2}=\left[\begin{array}{llll}\delta_{12} & \delta_{22} & \cdots & \delta_{N 2}\end{array}\right]^{\mathrm{T}}, \delta_{n}=\left[\begin{array}{llll}\delta_{1 n} & \delta_{2 n} & \cdots & \delta_{N n}\end{array}\right]^{\mathrm{T}}$ and $\delta=\left[\begin{array}{llll}\delta_{1}^{\mathrm{T}} & \delta_{2}^{\mathrm{T}} & \cdots & \delta_{n}^{\mathrm{T}}\end{array}\right]^{\mathrm{T}}$. Then, our main results on the synchronization of the states of all agents to the state of the leader agent can be stated in the following theorem 2, whose proof is presented in Section 3.3.2.

Theorem 2 Consider a system of $N$ agents whose dynamics are described by (3.1) and steered by the control protocol specified by (3.16), (3.19) and (3.20). Consider that the leader agent's dynamics are described by (3.2). Assume that the communication topology is represented by a switching connected undirected graph, and the leader agent is at least connected to one agent in the network. Suppose that Assumption 1 and Assumption 2 are satisfied. Then, given any bounded set $\mathcal{X}_{0}$, for any initial synchronization error $\delta(0)$ that belongs to $\mathcal{X}_{0}$, there exists a positive constant $c_{\mathcal{X}_{0}}$ such that the synchronization error $\delta$ is bounded as long as $c \geq c_{\mathcal{X}_{0}}$. Furthermore, the ultimate bound on the synchronization error $\delta$ can be reduced arbitrarily by increasing the value of the control parameter $c$.

\subsubsection{Theoretical Analysis}

To prove Theorem 2, we choose the Lyapunov function

$$
V=\frac{1}{2} m^{\mathrm{T}} m
$$


where $m=\left[m_{1} m_{2} \cdots m_{N}\right]^{\mathrm{T}}=\left(1+\frac{d}{d t}\right)^{n-1} \delta_{1}$ with $\delta_{1}$ defined in (3.21). Consequently,

$$
\begin{aligned}
m & =C_{n-1}^{0} \delta_{1}+C_{n-1}^{1} \delta_{2}+\cdots+C_{n-1}^{n-1} \delta_{n}, \\
\|m\| & \leq C_{n-1}^{0}\left\|\delta_{1}\right\|+C_{n-1}^{1}\left\|\delta_{2}\right\|+\cdots+C_{n-1}^{n-1}\left\|\delta_{n}\right\|,
\end{aligned}
$$

Denote $e_{1}=\left[\begin{array}{llll}e_{11} & e_{21} & \cdots & e_{N 1}\end{array}\right]^{\mathrm{T}}, e_{2}=\left[\begin{array}{llll}e_{12} & e_{22} & \cdots & e_{N 2}\end{array}\right]^{\mathrm{T}}, e_{n}=\left[\begin{array}{llll}e_{1 n} & e_{2 n} & \cdots & e_{N n}\end{array}\right]^{\mathrm{T}}$ and $e=\left[\begin{array}{llll}e_{1}^{\mathrm{T}} & e_{2}^{\mathrm{T}} & \cdots & e_{n}^{\mathrm{T}}\end{array}\right]^{\mathrm{T}}$.

In view of (3.17), (3.18), (3.21), we have

$$
\begin{aligned}
e_{1} & =-(L+B) \delta_{1}, \\
e_{2} & =-(L+B) \delta_{2}, \\
& \vdots \\
e_{n} & =-(L+B) \delta_{n}, \\
r & =C_{n-1}^{0} e_{1}+C_{n-1}^{1} e_{2}+\cdots+C_{n-1}^{n-1} e_{n}, \\
m & =C_{n-1}^{0} \delta_{1}+C_{n-1}^{1} \delta_{2}+\cdots+C_{n-1}^{n-1} \delta_{n} .
\end{aligned}
$$

Then it follows that

$$
r=-(L+B) m .
$$

Let $h_{0}>0$ be such that $\mathcal{X}_{0} \subseteq D_{0}=\left\{\delta:\left\|\delta_{1}\right\| \leq h_{0},\left\|\delta_{2}\right\| \leq h_{0}, \cdots,\left\|\delta_{n}\right\| \leq h_{0}\right\}$. We construct a level set $\Omega=\left\{\delta: V \leq \frac{1}{2} h^{2}\right\}$, where $h=2^{n-1} h_{0}$. It follows that $\mathcal{X}_{0} \subseteq \Omega$.

We now evaluate the derivative of $V$ within the level set $\Omega$ as follows,

$$
\dot{V}=m^{\mathrm{T}} \dot{m}
$$

where

$$
\begin{aligned}
\dot{m} & =\frac{d}{d t}\left\{C_{n-1}^{0} \delta_{1}+C_{n-1}^{1} \delta_{2}+\cdots+C_{n-1}^{n-1} \delta_{n}\right\} \\
& =\left(f(x)-\underline{f}\left(x_{0}, t\right)+u+w\right)+C_{n-1}^{0} \delta_{2}+C_{n-1}^{1} \delta_{3}+\cdots+C_{n-1}^{n-2} \delta_{n} \\
& =\left(W^{\mathrm{T}} \varphi(x)+\varepsilon-\underline{f}\left(x_{0}, t\right)+c r-\hat{W}^{\mathrm{T}} \varphi(x)+w\right)+C_{n-1}^{0} \delta_{2}+C_{n-1}^{1} \delta_{3}+\cdots+C_{n-1}^{n-2} \delta_{n} \\
& =\left(\tilde{W}^{\mathrm{T}} \varphi(x)+\varepsilon-\underline{f}\left(x_{0}, t\right)+c r+w\right)+C_{n-1}^{0} \delta_{2}+C_{n-1}^{1} \delta_{3}+\cdots+C_{n-1}^{n-2} \delta_{n} .
\end{aligned}
$$

Here, $\varepsilon \leq \varepsilon_{0}$ is the ideal RBFNN function approximation error of $f(x)$ as defined in Section 3.1, $\varphi(x)=$ $\left[\varphi_{1}\left(x_{1}\right) \varphi_{2}\left(x_{2}\right) \cdots \varphi_{N}\left(x_{N}\right)\right]^{\mathrm{T}}, \hat{f}(x)=\left[\begin{array}{lll}\hat{f}_{1}\left(x_{1}\right) & \hat{f}_{2}\left(x_{2}\right) \cdots & \hat{f}_{N}\left(x_{N}\right)\end{array}\right]^{\mathrm{T}}, \underline{f}\left(x_{0}, t\right)=f_{0}\left(x_{0}, t\right)\left[\begin{array}{llll}1 & 1 & \cdots & 1\end{array}\right]^{\mathrm{T}}$ and 
$\tilde{W}=\tilde{W}=W-\hat{W}$ is the neural network weight approximation error with $W=\operatorname{diag}\left\{W_{i}\right\}$ being the ideal neural network weights and $\hat{W}=\operatorname{diag}\left\{\hat{W}_{i}\right\}$.

Therefore, we have

$$
\begin{aligned}
\dot{V} & =m^{\mathrm{T}} \dot{m} \\
& =m^{\mathrm{T}}\left(\tilde{W}^{\mathrm{T}} \varphi(x)+\varepsilon-\underline{f}\left(x_{0}, t\right)+w\right)+m^{\mathrm{T}}\left(C_{n-1}^{0} \delta_{2}+C_{n-1}^{1} \delta_{3}+\cdots+C_{n-1}^{n-2} \delta_{n}\right) \\
& =m^{\mathrm{T}}\left(\tilde{W}^{\mathrm{T}} \varphi(x)+\varepsilon-\underline{f}\left(x_{0}, t\right)+c(-(L+B)) m+w\right)+m^{\mathrm{T}}\left(C_{n-1}^{0} \delta_{2}+C_{n-1}^{1} \delta_{3}+\cdots+C_{n-1}^{n-2} \delta_{n}\right) \\
& =-c m^{\mathrm{T}}(L+B) m+m^{\mathrm{T}}\left(\varepsilon-\underline{f}\left(x_{0}, t\right)+w\right)+m^{\mathrm{T}} \tilde{W}^{\mathrm{T}} \varphi(x)+m^{\mathrm{T}}\left(C_{n-1}^{0} \delta_{2}+C_{n-1}^{1} \delta_{3}+\cdots+C_{n-1}^{n-2} \delta_{n}\right)
\end{aligned}
$$

Since the communication topology is connected, we know that $L+B$ is positive definite. Therefore, we have

$\dot{V} \leq-\frac{1}{2} c \underline{\sigma}(L+B)\|m\|^{2}+\|m\|\left(\|\varepsilon\|+\left\|\underline{f}\left(x_{0}, t\right)\right\|+\|w\|+\|\tilde{W}\|\|\varphi(x)\|\right)+\|m\|\left(C_{n-1}^{0}\left\|\delta_{2}\right\|+C_{n-1}^{1}\left\|\delta_{3}\right\|+\cdots+C_{n-1}^{n-2}\left\|\delta_{n}\right\|\right)$.

Note that the designed neural network weights tuning law ensures that $\operatorname{Tr}\left\{\hat{W}_{i}^{\mathrm{T}} \hat{W}_{i}\right\}$ would be bounded for all $i$. This fact was established as Lemma 2 in [11]. Then it follows that $\tilde{W}_{i}$ is bounded for all $i$. Consequently, we have

$$
\dot{V} \leq-\frac{1}{2} c \underline{\sigma}(L+B)\|m\|^{2}+\|m\| B_{u}
$$

where $B_{u}=\varepsilon_{0}+F_{0}+L_{0} \Phi_{m}+\Delta_{0}+\left(2^{n-1}-1\right) M_{0}$ is a positive constant. Here $\varepsilon_{0}, F_{0}, L_{0}, \Phi_{m}$, and $\Delta_{0}$ respectively denote the bounds of $\varepsilon, \underline{f}\left(x_{0}, t\right), \tilde{W}, \varphi(x)$ and $w$. $M_{0}$ denotes an upper bound for all $\left\|e_{i}\right\|$, $i=2,3, \cdots, n$.

Let

$$
c_{\mathcal{X}_{0}}=\frac{2 B_{u}}{\sigma_{l} h}
$$

where $\sigma_{l}$ is as defined in Assumption 2. Then, for any $c \geq c_{\mathcal{X}_{0}}$, we have $\dot{V}<0$ as long as $\|m\|>h$. This implies that $\dot{V}<0$ as long as $V>\frac{1}{2} h^{2}$. Thus, the level set $\Omega$ is an invariant set for any $c \geq c \mathcal{X}_{0}$.

We have now proven that $\|m\|(t)$ is bounded as long as $c \geq c_{\mathcal{X}_{0}}$. Recall that $m=\left(1+\frac{d}{d t}\right)^{n-1} \delta_{1}=$ $C_{n-1}^{0} \delta_{1}+C_{n-1}^{1} \delta_{2}+\cdots+C_{n-1}^{n-1} \delta_{n}$. It follows from lemma 1 that $\left\|\delta_{1}(t)\right\|,\left\|\delta_{2}(t)\right\|, \cdots,\left\|\delta_{n}(t)\right\|$ are all bounded since $\left\|\delta_{1}(0)\right\|,\left\|\delta_{2}(0)\right\|, \cdots,\left\|\delta_{n}(0)\right\|$ are bounded. That is, the synchronization error $\delta$ is also bounded. Furthermore, it is easy to see that the ultimate bound of the synchronization error $\delta$ could be reduced arbitrarily by increasing the value of the control parameter $c$. 


\subsection{Synchronization Control of $n$ th-order Systems with Switching Strongly-connected Directed Graph Communication Topolo- gies}

We consider a network of $N$ agents. Each agent is a $n$ th-order system whose dynamics are described by (3.1). Besides the $N$ agents, there is also a leader agent, whose dynamics are described by (3.2).

The communication topology under consideration is switching and directed. Let $G=\left\{G_{1}, G_{2}, \cdots, G_{m}\right\}$ be a finite set of directed communication topologies, where for each $G_{k}$, the topology among the $N$ follower agents is fixed, both in connectivity and edge weights, and strongly-connected, and at least one follower agent in topology $G_{k}$ has access to the information of the leader. In this section, such a switching topology $G$ is called as a switching strongly-connected directed topology. Let $\sigma(t)$ denote the communication topology switching signal. For example, $\sigma(t)=i$ implies that the communication topology $G_{i}$ is active at time $t$. For any given $t \geq 0, \sigma(t) \in\{1,2, \cdots, m\}$. Let $t_{0}, t_{1}, t_{2}, \cdots$ be the time the communication topology switches, where $0=t_{0}<t_{1}<t_{2} \cdots$ can be an infinite sequence. In this section, we assume that the communication topology cannot switch infinitely fast, namely, there exists a constant dwell time $\tau>0$ such that $t_{i+1}-t_{i} \geq \tau$ for all $i$. With the notation above, the switching and directed communication topology under consideration in this section is denoted as $G_{\sigma(t)}$.

For each topology $G_{k} \in G, k=1,2, \cdots, m$, we denote the topology among the $N$ follower agents as $G_{k \text { Follower }}=\left(V, E_{k}\right)$, where $V=\left\{v_{1}, v_{2}, \cdots, v_{N}\right\}$ is a finite, nonempty set of $N$ vertices, each denoting an follower agent in the network, and $E_{k} \subset V \times V$ is the set of edges of the graph. An edge $\left(v_{i}, v_{j}\right)$ denotes that agent $j$ has access to the information of agent $i$. Define the adjacency matrix as $A=\left[a_{i j}\right]$ with $a_{i j}>0$ if $\left(v_{j}, v_{i}\right) \in E$ and $a_{i j}=0$ otherwise. The weighted in-degree matrix $D$ is defined as a diagonal matrix $D=\operatorname{diag}\left\{d_{1}, d_{2}, \cdots, d_{N}\right\}$ with $d_{i}=\sum_{j=1}^{N} a_{i j}, i=1,2, \cdots, N$. The graph Laplacian matrix is defined as $L=D-A$. The leader agent is labeled as $v_{0}$. An edge from the leader agent $v_{0}$ to follower agent $i$ denotes that agent $i$ has access to the information of the leader agent, with the communication weight defined as $b_{i}>0$. On the other hand, if a follower agent $i$ does not have access to the information of the leader agent, then $b_{i}=0$. In this section, we assume at least one follower agent has access to the information of the leader agent, namely, there is at least one $b_{i}>0$. Define matrix $B=\operatorname{diag}\left\{b_{1}, b_{2}, \cdots, b_{N}\right\}$. Note that matrix $A, D$ and $B$ are fixed for a given topology $G_{k}$. Some or all the matrices $A, D$ and $B$ are different for topologies $G_{k}$ and $G_{j}, k \neq j$. Note that, for each fixed topology $G_{k} \in G=\left\{G_{1}, G_{2}, \cdots, G_{m}\right\}$, the weight matrices $A$ and $B$ are constant. 
The following fact is important in the control design and the analysis of the closed-loop behaviors to be presented in this section. Recall that an M-matrix is a square matrix with all off-diagonal elements being non-positive and all principal minors being non-negative.

Lemma 4 ([54], [16], [14]) If a directed graph topology among the follower agents $G_{k \text { Follower }}$ is strongly connected and $B=\operatorname{diag}\left\{b_{1}, b_{2}, \cdots, b_{N}\right\}$ has at least one $b_{i}>0$, then $L+B$ is a non-singular M-matrix. It follows that $(L+B)^{T}$ is also a non-singular M-matrix. Define $s=\left[s_{1} s_{2} \cdots s_{N}\right]^{T}=\left((L+B)^{T}\right)^{-1} \underline{1}$, where 1 denotes the vector $[11 \cdots 1]^{T}$. Define matrix $S_{k}=\operatorname{diag}\left\{s_{1}, s_{2}, \cdots, s_{N}\right\}$. Then $S_{k}>0$ and $Q=(L+B)^{T} S_{k}+S_{k}\left((L+B)^{T}\right)^{T}=(L+B)^{T} S_{k}+S_{k}(L+B)>0$.

Based on Lemma 4, for each $G_{k} \in G, k=1,2, \cdots, m$, we can construct a positive definite matrix $S_{k}$. Let $\sigma_{\mathrm{L}}>0$ denote a lower bound of the minimal singular values of the matrices $S_{k}, k=1,2, \cdots, m$. Let $\sigma_{\mathrm{U}}>0$ denote a upper bound of the maximal singular values of the matrices $S_{k}, k=1,2, \cdots, m$.

In the remainder of this section, Section 3.4.1 provides the control algorithm description and main results, and Section 3.4.2 presents the proof of the main results.

\subsubsection{Control Protocols and the Closed-loop System Performance}

We propose the following distributed control laws for the follower agents to synchronize their states to the state of the leader agent,

$$
\begin{aligned}
u_{i} & =c r_{i}-\widehat{f}_{i}\left(x_{i}\right) \\
& =c r_{i}-\widehat{W}_{i}^{\mathrm{T}} \varphi_{i}\left(x_{i}\right), i=1,2, \cdots, N,
\end{aligned}
$$

where

$$
\begin{aligned}
r_{i} & =\left(1+\frac{d}{d t}\right)^{n-1} e_{i 1} \\
& =C_{n-1}^{0} e_{i 1}+C_{n-1}^{1} e_{i 2}+\cdots+C_{n-1}^{n-1} e_{i n}
\end{aligned}
$$

with

$$
e_{i k}=\sum_{j \in N_{i}} a_{i j}(t)\left(x_{j k}-x_{i k}\right)+b_{i}(t)\left(x_{0 k}-x_{i k}\right) .
$$

In $(3.27)-(3.29), C_{i}^{j}$ 's are coefficients of the binomial expansion, $c>0$ is a control gain parameter, $\varphi_{i}\left(x_{i}\right)=$ $\left[\varphi_{i 1}\left(x_{i}\right) \varphi_{i 2}\left(x_{i}\right) \cdots \varphi_{i \eta}\left(x_{i}\right)\right]^{\mathrm{T}}$ are the activation functions of the neural network with $\eta$ being the number of neurons, and $\widehat{W}_{i}=\left[\widehat{W}_{i 1} \widehat{W}_{i 2} \cdots \widehat{W}_{i \eta}\right]^{\mathrm{T}}$ are the neural network weights, and $a_{i j}(t)$ and $b_{i}(t)$ are the constant 
graph parameters $\left(a_{i j}\right.$ and $\left.b_{i}\right)$ corresponding to the current active communication topology $G_{\sigma(t)}$, as defined in Section 3.4.

The neural network weights $\widehat{W}_{i}$ are updated according to

$$
\dot{\widehat{W}}_{i}= \begin{cases}-\chi_{i} \varphi_{i} r_{i}+\chi_{i} \frac{r_{i} \widehat{W}_{i}^{\mathrm{T}} \varphi_{i}}{\operatorname{Tr}\left\{\widehat{W}_{i}^{\mathrm{T}} \widehat{W}_{i}\right\}} \widehat{W}_{i}, & \text { if }-r_{i} \widehat{W}_{i}^{\mathrm{T}} \varphi_{i}>0 \text { and } \\ -\chi_{i} \varphi_{i} r_{i}, & \operatorname{Tr}\left\{\widehat{W}_{i}^{\mathrm{T}} \widehat{W}_{i}\right\}=W_{\max i} \\ & \text { otherwise }\end{cases}
$$

with the initial value of the neural network weight matrix being chosen to satisfy

$$
\operatorname{Tr}\left(\widehat{W}_{i}^{\mathrm{T}}(0) \widehat{W}_{i}(0)\right) \leq W_{\max i}
$$

Here, $W_{\max i}$ is a given positive constant for limiting the neural network weights, and $\chi_{i}>0$ is a given constant which controls the adaption rate of $\widehat{W}_{i}$. This neural network weight matrix updating law is adapted from [11]. Note that, Lemma 3 guarantees the boundedness of $\widehat{W}_{i}$.

Define the synchronization error as

$$
\delta_{i k}=x_{i k}-x_{0 k}, \quad k=1,2, \cdots, n, i=1,2, \cdots, N .
$$

Denote $\delta_{1}=\left[\begin{array}{llll}\delta_{11} & \delta_{21} & \cdots & \delta_{N 1}\end{array}\right]^{\mathrm{T}}, \delta_{2}=\left[\begin{array}{llll}\delta_{12} & \delta_{22} & \cdots & \delta_{N 2}\end{array}\right]^{\mathrm{T}}, \cdots, \delta_{n}=\left[\begin{array}{llll}\delta_{1 n} & \delta_{2 n} & \cdots & \delta_{N n}\end{array}\right]^{\mathrm{T}}$, and $\delta=\left[\begin{array}{llll}\delta_{1}^{\mathrm{T}} & \delta_{2}^{\mathrm{T}} & \cdots & \delta_{n}^{\mathrm{T}}\end{array}\right]^{\mathrm{T}}$.

We state our main results in this section in a theorem as follows. The proof of this theorem is provided in Section 3.4.2.

Theorem 3 Consider a multi-agent system of $N$ follower agents whose dynamics are described by (3.1) and steered by the control protocols specified by (3.27), (3.30) and (3.31). Let the dynamics of the leader agent be described by (3.2). Let the communication topology among the follower agents be represented by a switching strongly-connected directed graph, and at least one follower agent has access to the information of the leader agent, as described in Section 3.4 above. Then, for any given bounded set $\mathcal{X}_{0} \subset R^{(N+1) \times n}$, there exists a positive constant $c_{\mathcal{X}_{0}}$ such that, for any initial states $x(0)=\left[x_{0}(0) x_{1}(0) \cdots x_{N}(0)\right]^{T} \in \mathcal{X}_{0}$, the synchronization error $\delta$ is ultimately bounded as long as $c \geq c_{\mathcal{X}_{0}}$. Furthermore, the ultimate bound on the synchronization error $\delta$ can be reduced arbitrarily by increasing the value of the control gain parameter $c$.

Theorem 3 extends the previous results in Section 3.2 and Section 3.3 to communication topology which is represented by a switching strongly-connected directed graph. The communication topologies of many real-world engineering systems can be both directed and switching, such as a mobile multi-robotic system 
with nonidentical sensing radius. Therefore, the results in Theorem 3 are applicable to more real-world engineering systems.

\subsubsection{Theoretical Analysis}

In Section 3.2 and Section 3.3, we employed a common Lyapunov function approach to establishing our results for a fixed strongly-connected directed graph topology and switching connected undirected graph topologies. It is not clear how a common Lyapunov function can be constructed for the situation of a switching strongly-connected directed graph communication topology. To overcome this difficulty, we construct in this section a sequence of different Lyapunov functions and use them to prove our results in Theorem 3.

Let

$$
\begin{aligned}
\Delta & =\left(1+\frac{d}{d t}\right)^{n-1} \delta_{1} \\
& =C_{n-1}^{0} \delta_{1}+C_{n-1}^{1} \delta_{2}+\cdots+C_{n-1}^{n-1} \delta_{n} .
\end{aligned}
$$

Denote $r_{G_{k}}=-\left(L_{G_{k}}+B_{G_{k}}\right) \Delta$, where $L_{G_{k}}$ and $B_{G_{k}}$ are the corresponding matrices $L$ and $B$ for graph $G_{k} \in G$, as defined in Section 3.4. Then, we construct a sequence of $m$ different Lyapunov functions,

$$
\begin{aligned}
V_{k}(\Delta) & =\frac{1}{2} r_{G_{k}}^{\mathrm{T}} S_{k} r_{G_{k}}, \\
& =\frac{1}{2}\left(\left(L_{G_{k}}+B_{G_{k}}\right) \Delta\right)^{\mathrm{T}} S_{k}\left(\left(L_{G_{k}}+B_{G_{k}}\right) \Delta\right), k=1,2, \cdots, m,
\end{aligned}
$$

where $S_{k}$ is the corresponding positive definite matrix constructed from $G_{k} \in G$ in the way specified by Lemma 4.

Recall that $\sigma_{\mathrm{L}}>0$ is a lower bound of the minimal singular values of the diagonal matrices $S_{k}, k=$ $1,2, \cdots, m$, and $\sigma_{\mathrm{U}}>0$ is a upper bound of the maximal singular values of the diagonal matrices $S_{k}$, $k=1,2, \cdots, m$. Since the communication topology among the follower agents is strongly connected, $L_{G_{k}}+B_{G_{k}}$ is a non-singular matrix. Denote $z_{\mathrm{L}}>0$ as a lower bound of the minimal singular values of the matrix $L_{G_{k}}+B_{G_{k}}, k=1,2, \cdots, m$, and denote $z_{\mathrm{U}}>0$ as a upper bound of the maximal singular values of the matrix $L_{G_{k}}+B_{G_{k}}, k=1,2, \cdots, m$. Then we have,

$$
\frac{1}{2} \sigma_{\text {Lower }}\|\Delta\|^{2} \leq V_{k} \leq \frac{1}{2} \sigma_{\text {Upper }}\|\Delta\|^{2}, k=1,2, \cdots, m
$$

where $\sigma_{\text {Lower }}=\sigma_{\mathrm{L}} z_{\mathrm{L}}^{2}$ and $\sigma_{\mathrm{Upper}}=\sigma_{\mathrm{U}} z_{\mathrm{U}}^{2}$. 
Without loss of generality, we can assume that the topologies $G_{1}, G_{2}, \cdots, G_{m}$ are labeled in the same order as the first time they are active in the switching communication topology. Namely, if we denote $T_{k}$ as the first time that $G_{k}$ is active, then $T_{1}<T_{2}<\cdots<T_{m}$.

Consider the ball $B_{0}=\{\Delta:\|\Delta\| \leq h\}$, where $h>0$ is a constant such that $x(0) \in \mathcal{X}_{0}$ implies that $\Delta(0) \in B_{0}$. Construct $m$ level sets $R_{k}=\left\{\Delta: V_{k}(\Delta) \leq h_{k}\right\}$, where $h_{k}>0$, such that the ball $B_{0}$ is contained by the intersection of all these $R_{k}, k=1,2, \cdots, m$. Note that, these sets $R_{k}$ are constructed in a way such that $R_{i}$ is contained in the set $R_{i+1}, i=m-1, \cdots, 2,1$. Denote the union of all these $m$ level sets $R_{k}$ as set $R$, which is obviously equal to $R_{m}$. Define a ball $B_{\infty}=\left\{\Delta:\|\Delta\| \leq \frac{1}{2} h\right\}$. Then, to prove our results in Theorem 3, we will first establish the following lemma.

Lemma 5 As long as the control gain $c$ is greater than some constant $c_{\mathcal{X}_{0}}$, any trajectory of $\Delta$ starting from the ball $B_{0}$ always belongs to the set $R_{\sigma(t)}$, and will enter and stay inside the smaller ball $B_{\infty}$ in a finite time. Moreover, such a ball $B_{\infty}$ can be chosen to be arbitrarily small as long as we increase the value of the control gain $c$.

We will prove Lemma 5 as follows.

To start with, we conduct the Lyapunov analysis in a given time interval $\left[t_{i}, t_{i+1}\right)$, where $t_{i}$ and $t_{i+1}$ are contiguous topology switching times. Note that, in such a time interval, the communication topology is fixed. Denote the corresponding constructed positive definite matrix in this given time interval as $S=S_{\sigma\left(t_{i}\right)} \in\left\{S_{1}, S_{2}, \cdots, S_{m}\right\}$, and denote the corresponding topology graph parameter matrices in this given interval as $L=L_{G_{\sigma\left(t_{i}\right)}}$ and $B=B_{G_{\sigma\left(t_{i}\right)}}$, where $\sigma(t)$ is the communication topology switching signal as defined in Section 3.4. Denote $r=\left[r_{1} r_{2} \cdots r_{N}\right]^{\mathrm{T}}$, where $r_{i}, i=1,2, \cdots, N$, is defined in (3.5). In view of (3.28), (3.29), (3.32) and (3.33), it is easy to see that, in this given time interval $\left[t_{i}, t_{i+1}\right)$, $r=-\left(L_{G_{\sigma\left(t_{i}\right)}}+B_{G_{\sigma\left(t_{i}\right)}}\right) \Delta=-(L+B) \Delta$. Then, the corresponding Lyapunov function in this time interval $V=V_{\sigma\left(t_{i}\right)}$ is expressed as

$$
\begin{aligned}
V(\Delta) & =\frac{1}{2}((L+B) \Delta)^{\mathrm{T}} S((L+B) \Delta) \\
& =\frac{1}{2} r^{\mathrm{T}} S r .
\end{aligned}
$$

Assume that $\Delta\left(t_{i}\right) \in R_{1}, \Delta\left(t_{i}\right) \in R_{2}, \cdots, \Delta\left(t_{i}\right) \in R_{m}$. This assumption can always hold for $t_{i}=t_{0}$ by construction of the sets $R_{k}$. Later in this section we will prove that $\Delta\left(t_{k}\right) \in R_{1}, \Delta\left(t_{k}\right) \in R_{2}, \cdots, \Delta\left(t_{k}\right) \in R_{m}$ indeed holds for all $t_{k}, k=0,1,2 \cdots$, as long as the control gain $c$ is greater than some constant $c_{\mathcal{X}_{0}}$.

$$
\text { Let } e_{1}=\left[\begin{array}{llll}
e_{11} & e_{21} & \cdots & e_{N 1}
\end{array}\right]^{\mathrm{T}}, e_{2}=\left[\begin{array}{llll}
e_{12} & e_{22} & \cdots & e_{N 2}
\end{array}\right]^{\mathrm{T}}, \cdots, e_{n}=\left[\begin{array}{llll}
e_{1 n} & e_{2 n} & \cdots & e_{N n}
\end{array}\right]^{\mathrm{T}} \text {, and } e=\left[\begin{array}{llll}
e_{1}^{\mathrm{T}} & e_{2}^{\mathrm{T}} & \cdots & e_{n}^{\mathrm{T}}
\end{array}\right]^{\mathrm{T}} \text {. }
$$


In view of (3.28), (3.29) and (3.32), we have

$$
e_{k}=-(L+B) \delta_{k}, \quad k=1,2, \cdots, n,
$$

and

$$
r=C_{n-1}^{0} e_{1}+C_{n-1}^{1} e_{2}+\cdots+C_{n-1}^{n-1} e_{n}
$$

We note here that

$$
\dot{e}_{k}=e_{k+1}, \quad k=1,2, \cdots, n-1,
$$

and

$$
\dot{e}_{n}=-(L+B)\left(F\left(x_{1}, x_{2}, \cdots, x_{N}\right)+u+w-F_{0}\left(x_{0}\right)\right) \text {, }
$$

where

$$
\begin{gathered}
F\left(x_{1}, x_{2}, \cdots, x_{N}\right)=\left[\begin{array}{c}
f_{1}\left(x_{1}\right) \\
f_{2}\left(x_{2}\right) \\
\vdots \\
f_{N}\left(x_{N}\right)
\end{array}\right], \\
F_{0}\left(x_{0}, t\right)=\left[\begin{array}{c}
f_{0}\left(x_{0}, t\right) \\
f_{0}\left(x_{0}, t\right) \\
\vdots \\
f_{0}\left(x_{0}, t\right)
\end{array}\right],
\end{gathered}
$$

$u=\left[\begin{array}{llll}u_{1} & u_{2} & \cdots & u_{N}\end{array}\right]^{\mathrm{T}}$ and $w=\left[\begin{array}{llll}w_{1} & w_{2} & \cdots & w_{N}\end{array}\right]^{\mathrm{T}}$.

Since the communication topology among the follower agents is strongly connected, $L+B$ is a non-singular matrix, and hence

$$
\left\|e_{k}\right\| \leq \bar{\sigma}(L+B)\left\|\delta_{k}\right\|, \quad k=1,2, \cdots, n
$$

and

$$
\left\|\delta_{k}\right\| \leq \frac{\left\|e_{k}\right\|}{\underline{\sigma}(L+B)}, \quad k=1,2, \cdots, n .
$$

Here and later in this proof, for a non-singular matrix $M, \bar{\sigma}(M)$ and $\underline{\sigma}(M)$ denote the maximal and minimal singular values of $M$, respectively.

Consequently, we have

$$
\|r\| \leq C_{n-1}^{0} \bar{\sigma}(L+B)\left\|\delta_{1}\right\|+C_{n-1}^{1} \bar{\sigma}(L+B)\left\|\delta_{2}\right\|+\cdots+C_{n-1}^{n-1} \bar{\sigma}(L+B)\left\|\delta_{n}\right\| .
$$


To evaluate the derivative of $V$ along the closed-loop trajectories within the corresponding level set $R_{\sigma\left(t_{i}\right)}$ in time interval $\left[t_{i}, t_{i+1}\right)$, we first evaluate the derivative of $r$ along the closed-loop trajectories within the level set $R_{\sigma\left(t_{i}\right)}$ as follows,

$$
\begin{aligned}
\dot{r} & =C_{n-1}^{0} \dot{e}_{1}+C_{n-1}^{1} \dot{e}_{2}+\cdots+C_{n-1}^{n-1} \dot{e}_{n} \\
& =C_{n-1}^{0} e_{2}+C_{n-1}^{1} e_{3}+\cdots+C_{n-1}^{n-2} e_{n}-(L+B)\left(F\left(x_{1}, x_{2}, \cdots, x_{N}\right)-F_{0}\left(x_{0}, t\right)+u+w\right) \\
& =C_{n-1}^{0} e_{2}+C_{n-1}^{1} e_{3}+\cdots+C_{n-1}^{n-2} e_{n}-(L+B)\left(W^{\mathrm{T}} \varphi\left(x_{1}, x_{2}, \cdots, x_{N}\right)+\varepsilon-F_{0}\left(x_{0}, t\right)+c r-\widehat{W}^{\mathrm{T}} \varphi\left(x_{1}, x_{2}, \cdots, x_{N}\right)+w\right) \\
& =C_{n-1}^{0} e_{2}+C_{n-1}^{1} e_{3}+\cdots+C_{n-1}^{n-2} e_{n}-(L+B)\left(\widetilde{W}^{\mathrm{T}} \varphi\left(x_{1}, x_{2}, \cdots, x_{N}\right)+\varepsilon-F_{0}\left(x_{0}, t\right)+c r+w\right) .
\end{aligned}
$$

In the above derivation, $\varphi\left(x_{1}, x_{2}, \cdots, x_{N}\right)=\left[\varphi_{1}\left(x_{1}\right) \varphi_{2}\left(x_{2}\right) \cdots \varphi_{N}\left(x_{N}\right)\right]^{\mathrm{T}}, W=\operatorname{diag}\left\{W_{1}, W_{2}, \cdots, W_{N}\right\}$, $\widehat{W}=\operatorname{diag}\left\{\widehat{W}_{1}, \widehat{W}_{2}, \cdots, \widehat{W}_{N}\right\}, \widetilde{W}=\widehat{W}-W, \varepsilon=\left[\varepsilon_{1} \varepsilon_{2} \cdots \varepsilon_{N}\right]^{\mathrm{T}}$, and $\left(\varphi_{i}\left(x_{i}\right), W_{i}\right)$ is the neural network approximation of $f_{i}\left(x_{i}\right)$ such that $f_{i}\left(x_{i}\right)=W_{i}^{\mathrm{T}} \varphi_{i}\left(x_{i}\right)+\varepsilon_{i}$ with $\left|\varepsilon_{i}\right| \leq \varepsilon_{0}$ for some $\varepsilon_{0}>0$ on the compact set $R$.

Therefore, the derivative of $V$ along the closed-loop trajectories within the level set $R_{\sigma\left(t_{i}\right)}$ can be evaluated as follows,

$$
\begin{aligned}
\dot{V}= & r^{\mathrm{T}} S \dot{r} \\
= & -r^{\mathrm{T}} S(L+B)\left(\widetilde{W}^{\mathrm{T}} \varphi\left(x_{1}, x_{2}, \cdots, x_{N}\right)+\varepsilon-F_{0}\left(x_{0}, t\right)+c r+w\right)+r^{\mathrm{T}} S\left(C_{n-1}^{0} e_{2}+C_{n-1}^{1} e_{3}+\cdots+C_{n-1}^{n-2} e_{n}\right) \\
= & -c r^{\mathrm{T}} S(L+B) r-r^{\mathrm{T}} S(L+B)\left(\varepsilon-F_{0}\left(x_{0}, t\right)+w\right)-r^{\mathrm{T}} S(L+B) \widetilde{W}^{\mathrm{T}} \varphi\left(x_{1}, x_{2}, \cdots, x_{N}\right) \\
& +r^{\mathrm{T}} S\left(C_{n-1}^{0} e_{2}+C_{n-1}^{1} e_{3}+\cdots+C_{n-1}^{n-2} e_{n}\right) .
\end{aligned}
$$

Since the communication topology among the follower agents is strongly connected, by Lemma 4, the matrix $Q=S(L+B)+(L+B)^{\mathrm{T}} S$ is positive definite. Therefore, we have

$$
\begin{aligned}
\dot{V}= & -\frac{c}{2} r^{\mathrm{T}} Q r-r^{\mathrm{T}} S(L+B)\left(\varepsilon-F_{0}\left(x_{0}, t\right)+w\right)-r^{\mathrm{T}} S(L+B) \widetilde{W}^{\mathrm{T}} \varphi\left(x_{1}, x_{2}, \cdots, x_{N}\right) \\
& +r^{\mathrm{T}} S\left(C_{n-1}^{0} e_{2}+C_{n-1}^{1} e_{3}+\cdots+C_{n-1}^{n-2} e_{n}\right) \\
\leq & -\frac{c}{2} \underline{\sigma}(Q)\|r\|^{2}+\|r\| \bar{\sigma}(S) \bar{\sigma}(L+B)\left(\|\varepsilon\|+\left\|F_{0}\left(x_{0}, t\right)\right\|+\|w\|+\|\widetilde{W}\|\left\|\varphi\left(x_{1}, x_{2}, \cdots, x_{N}\right)\right\|\right) \\
& +\|r\| \bar{\sigma}(S)\left(C_{n-1}^{0}\left\|e_{2}\right\|+C_{n-1}^{1}\left\|e_{3}\right\|+\cdots+C_{n-1}^{n-2}\left\|e_{n}\right\|\right) .
\end{aligned}
$$

Note that, by Lemma 3, the neural network weights tuning law (3.30) and (3.31) ensures that $\operatorname{Tr}\left\{\widehat{W}^{\mathrm{T}} \widehat{W}\right\}$ is bounded for all time. It follows that $\widetilde{W}$ is bounded for all time. Assume that $\|\widetilde{W}\| \leq B_{\widetilde{W}}$ for some $B_{\widetilde{W}}>0$.

By the assumption of the boundedness of trajectories of the leader agent, $F_{0}\left(x_{0}, t\right)$ is bounded on $R$. 
Assume that $\left\|F_{0}\left(x_{0}, t\right)\right\| \leq B_{F_{0}}$ for some $B_{F_{0}}>0$. Recall that, $w$ and $\varphi\left(x_{1}, x_{2}, \cdots, x_{N}\right)$ are both bounded. Let $\|w\| \leq B_{w}$ for some $B_{w}>0$ and $\left\|\varphi\left(x_{1}, x_{2}, \cdots, x_{N}\right)\right\| \leq B_{\varphi}$ for some $B_{\varphi}>0$. Also, by Lemma $1, e_{i}$, $i=1,2, \cdots, n$, are bounded on $R$. Let $\left\|e_{i}\right\| \leq B_{e}, i=1,2, \cdots, n$, for some $B_{e}>0$. Then, we have

$$
\begin{aligned}
\dot{V} & \leq-\frac{c}{2} \frac{\sigma}{}(Q)\|r\|^{2}+\bar{B}\|r\| \\
& \leq-c \frac{\underline{\sigma}(Q)}{\bar{\sigma}(S)} V+\frac{\sqrt{2} \bar{B}}{\sqrt{\underline{\sigma}(S)}} V^{\frac{1}{2}} \\
& \leq-c_{1} \frac{\underline{\sigma}(Q)}{\bar{\sigma}(S)} V-\left(c-c_{1}\right) \frac{\underline{\sigma}(Q)}{\bar{\sigma}(S)} V+\frac{\sqrt{2} \bar{B}}{\sqrt{\underline{\sigma}(S)}} V^{\frac{1}{2}} \\
& =-c_{1} \frac{\underline{\sigma}(Q)}{\bar{\sigma}(S)} V^{\frac{1}{2}}\left(V^{\frac{1}{2}}-\frac{\sqrt{2} \bar{B} \bar{\sigma}(S)}{c_{1} \sqrt{\underline{\sigma}(S)} \underline{\sigma}(Q)}\right)-\left(c-c_{1}\right) \frac{\underline{\sigma}(Q)}{\bar{\sigma}(S)} V,
\end{aligned}
$$

where

$$
\bar{B}=\bar{\sigma}(S) \bar{\sigma}(L+B)\left(\sqrt{N} \varepsilon_{0}+B_{F_{0}}+B_{w}+B_{\widetilde{W}} B_{\varphi}\right)+\left(2^{n-1}-1\right) \bar{\sigma}(S) B_{e},
$$

and $c_{1} \in(0, c)$ is a constant parameter whose value is to be determined later.

For any given $\alpha \in(0,1)$, let

$$
c_{\mathcal{X}_{0}}=\underset{G_{\sigma\left(t_{i}\right)} \in G}{\operatorname{argmax}}\left[\frac{6 \bar{B} \bar{\sigma}(S) \sqrt{\sigma_{\text {Upper }}}}{\alpha \sqrt{\underline{\sigma}(S)} \underline{\sigma}(Q) h \sigma_{\text {Lower }}}+\frac{\bar{\sigma}(S)\left(\log \left(2 \sigma_{\text {Upper }}^{2}\right)-\log \left(\sigma_{\text {Lower }}^{2}\right)\right)}{\underline{\sigma}(Q) \tau}\right] .
$$

Here, we use the notation $\underset{\sigma}{\operatorname{argmax}}$ since $\bar{B}, S$ and $Q$ are constructed based on the corresponding graph $G_{\sigma\left(t_{i}\right)}$. $G_{\sigma\left(t_{i}\right)} \in G$

Then, for any $c \geq c_{\mathcal{X}_{0}}$, by choosing $c_{1}=\frac{6 \bar{B} \bar{\sigma}(S) \sqrt{\sigma_{\mathrm{Upper}}}}{\alpha \sqrt{\underline{\sigma(S)} \underline{\sigma}(Q) h \sigma_{\text {Lower }}}}$, we have

$$
\dot{V} \leq-\frac{\log \left(2 \sigma_{\text {Upper }}^{2}\right)-\log \left(\sigma_{\text {Lower }}^{2}\right)}{\tau} V<0
$$

for

$$
\Delta \in\left\{\Delta: \frac{\alpha^{2}}{16} \frac{\sigma_{\text {Lower }}^{2}}{\sigma_{\text {Upper }}} h^{2} \leq V(\Delta) \leq h_{\sigma\left(t_{i}\right)}\right\}
$$

where $\sigma\left(t_{i}\right)$ is the topology switching signal.

Recall that $t_{i+1}-t_{i}>\tau$. Then it follows from the above analysis that either $V\left(\Delta\left(t_{i+1}\right)\right)<\frac{\sigma_{\mathrm{Lower}}^{2}}{2 \sigma_{\mathrm{Upper}}^{2}} V\left(\Delta\left(t_{i}\right)\right)$ or $V\left(\Delta\left(t_{i+1}\right)\right)<\frac{\alpha^{2}}{16} \frac{\sigma_{\text {Lower }}^{2}}{\sigma_{\text {Upper }}} h^{2}$ would be true. Recall that, here $V\left(\Delta\left(t_{i+1}\right)\right)=V_{\sigma\left(t_{i}\right)}\left(\Delta\left(t_{i+1}\right)\right)$.

Then consider the values of all the other Lyapunov functions $V_{k}(\Delta)$ at time $t=t_{i+1}, k=1,2, \cdots, m$, $k \neq \sigma\left(t_{i}\right)$. 
If $V_{\sigma\left(t_{i}\right)}\left(t_{i+1}\right)<\frac{\sigma_{\mathrm{Lower}}^{2}}{2 \sigma_{\mathrm{Upper}}^{2}} V_{\sigma\left(t_{i}\right)}\left(t_{i}\right)$ is true, then we have

$$
\begin{aligned}
V_{k}\left(\Delta\left(t_{i+1}\right)\right) & \leq \frac{\sigma_{\text {Upper }}}{\sigma_{\text {Lower }}} V_{\sigma\left(t_{i}\right)}\left(\Delta\left(t_{i+1}\right)\right) \\
& \leq \frac{\sigma_{\text {Lower }}^{2}}{2 \sigma_{\text {Upper }}^{2}} \frac{\sigma_{\text {Upper }}}{\sigma_{\text {Lower }}} V_{\sigma\left(t_{i}\right)}\left(\Delta\left(t_{i}\right)\right) \\
& \leq \frac{\sigma_{\text {Lower }}^{2}}{2 \sigma_{\text {Upper }}^{2}} \frac{\sigma_{\text {Upper }}^{2}}{\sigma_{\text {Lower }}^{2}} V_{k}\left(\Delta\left(t_{i}\right)\right) \\
& =\frac{1}{2} V_{k}\left(\Delta\left(t_{i}\right)\right) .
\end{aligned}
$$

Recall that $\Delta\left(t_{i}\right) \in R_{1}, \Delta\left(t_{i}\right) \in R_{2}, \cdots, \Delta\left(t_{i}\right) \in R_{m}$. It follows directly in this case that, $\Delta\left(t_{i+1}\right) \in$ $R_{1}, \Delta\left(t_{i+1}\right) \in R_{2}, \cdots, \Delta\left(t_{i+1}\right) \in R_{m}$

On the other hand, if $V_{\sigma\left(t_{i}\right)}\left(t_{i+1}\right)<\frac{\alpha^{2}}{16} \frac{\sigma_{\text {Lower }}^{2}}{\sigma_{\text {Upper }}} h^{2}$ is true, we would have

$$
V_{k}\left(\Delta\left(t_{i+1}\right)\right) \leq \frac{\sigma_{\text {Upper }}}{\sigma_{\text {Lower }}} V_{\sigma\left(t_{i}\right)}\left(\Delta\left(t_{i+1}\right)\right) \leq \frac{\alpha^{2}}{16} \sigma_{\text {Lower }} h^{2}, k=1,2, \cdots, m
$$

In view of $(3.35)$, it follows in this case that the trajectory $\Delta$ has already entered the smaller ball $B_{\infty}=\left\{\Delta:\|\Delta\| \leq \frac{\alpha}{2} h\right\}$. Note that $B_{\infty} \subset R_{k}$, for each $k \in 1,2, \cdots, m$. Therefore, in this case we also have that $\Delta\left(t_{i+1}\right) \in R_{1}, \Delta\left(t_{i+1}\right) \in R_{2}, \cdots, \Delta\left(t_{i+1}\right) \in R_{m}$.

Based on the analysis above, we can conclude that $\Delta(t)$ belongs to the level set $R_{\sigma\left(t_{i}\right)}$ for $t \in\left[t_{i}, t_{i+1}\right)$, and that $\Delta\left(t_{i}\right) \in R_{1}, \Delta\left(t_{i}\right) \in R_{2}, \cdots, \Delta\left(t_{i}\right) \in R_{m}$ implies $\Delta\left(t_{i+1}\right) \in R_{1}, \Delta\left(t_{i+1}\right) \in R_{2}, \cdots, \Delta\left(t_{i+1}\right) \in R_{m}$. Next, the same Lyapunov analysis can be carried out within the level set $R_{\sigma\left(t_{i+1}\right)}$ for time intervals $\left[t_{i+1}, t_{i+2}\right)$ using the corresponding Lyapunov functions $V_{\sigma\left(t_{i+1}\right)}$ to conclude that $\Delta(t)$ belongs to the level set $R_{\sigma\left(t_{i+1}\right)}$ for $t \in\left[t_{i+1}, t_{i+2}\right)$, and $\Delta\left(t_{i+2}\right) \in R_{1}, \Delta\left(t_{i+2}\right) \in R_{2}, \cdots, \Delta\left(t_{i+2}\right) \in R_{m}$. The same analysis can be repeated for time intervals $\left[t_{i+2}, t_{i+3}\right),\left[t_{i+3}, t_{i+4}\right), \cdots$. We thus establish that any trajectory of $\Delta$ starting from the ball $B_{0}$ always belongs to the set $R_{\sigma(t)}$ as long as the control gain $c$ is greater than some constant $c_{\mathcal{X}_{0}}$.

Besides, in view of $(3.35),(3.40),(3.41)$ and $(3.42)$, we can also easily conclude that any trajectory of $\Delta$ starting from $B_{0}$ will finally enter and remain in the smaller ball $B_{\infty}=\left\{\Delta:\|\Delta\| \leq \frac{\alpha}{2} h\right\}$ in a finite time. Since $\alpha \in(0,1)$ is arbitrary, $B_{\infty}$ could be made to be arbitrarily small by decreasing the value of $\alpha$. A smaller value of $\alpha$ entails a larger value of $c_{\mathcal{X}_{0}}$ and hence a larger value of control gain parameter $c$. This completes the proof of Lemma 5 .

We have now proven that $\Delta(t)$ is bounded all the time as long as $c \geq c_{\mathcal{X}_{0}}$. We have also proven that the ultimate bound of $\Delta$ can be reduced arbitrarily by increasing the value of the control gain parameter c. Next, we apply Lemma 1 to establish the ultimate boundedness of the synchronization error $\delta$. To this 
end, we recall that $\Delta=\left(1+\frac{d}{d t}\right)^{n-1} \delta_{1}=C_{n-1}^{0} \delta_{1}+C_{n-1}^{1} \delta_{2}+\cdots+C_{n-1}^{n-1} \delta_{n}$. Then, by using Lemma 1 , we can conclude that $\left\|\delta_{1}(t)\right\|,\left\|\delta_{2}(t)\right\|, \cdots$ and $\left\|\delta_{n}(t)\right\|$ are all ultimately bounded, and their ultimate bounds can be reduced arbitrarily by increasing the value of $c$. Namely, the synchronization error $\delta$ is ultimately bounded, and their ultimate bounds can be reduced arbitrarily.

This completes the proof of Theorem 3 .

\subsection{Simulation Results}

In this section, simulation results are provided to demonstrate the effectiveness of our proposed control protocols. In particular, we consider a network of five third-order systems,

$$
\left\{\begin{array}{l}
\dot{x}_{11}=x_{12} \\
\dot{x}_{12}=x_{13} \\
\dot{x}_{13}=-2 x_{13}-3 \sin x_{12}+4 \cos x_{11}+u_{1}+w_{1} \\
\dot{x}_{21}=x_{22} \\
\dot{x}_{22}=x_{23} \\
\dot{x}_{23}=-6 x_{23}-8 \sin x_{22}+10 \cos x_{11}+u_{2}+w_{2} \\
\dot{x}_{31}=x_{32} \\
\dot{x}_{32}=x_{33} \\
\dot{x}_{33}=-12 x_{33}-15 \sin x_{32}+18 \cos x_{31}+u_{3}+w_{3} \\
\dot{x}_{41}=x_{42}, \\
\dot{x}_{42}=x_{43}, \\
\dot{x}_{43}=-20 x_{43}-24 \sin x_{42}+28 \cos x_{41}+u_{4}+w_{4} \\
\dot{x}_{51}=x_{52}, \\
\dot{x}_{52}=x_{53}, \\
\dot{x}_{53}=-42 x_{53}-48 \sin x_{52}+54 \cos x_{51}+u_{5}+w_{5}
\end{array}\right.
$$

where the disturbance $w_{i}=2 \sin \left(t+\frac{i \Pi}{8}\right), i=1,2, \cdots, 5$.

The leader agent is taken as a third-order system which has the following dynamics,

$$
\left\{\begin{array}{l}
\dot{x}_{01}=x_{02}, \\
\dot{x}_{02}=x_{03} \\
\dot{x}_{03}=-3 x_{01}-3 x_{02}-4 x_{03}+11 \cos (3 t)+15 \sin (3 t) .
\end{array}\right.
$$


Then, for such a multi-agent network system, the simulation results for the case of a fixed directed interaction topology are presented in Section 3.5.1. In Section 3.5.2, we provide the simulation results for the case of switching undirected interaction topology. Finally, the simulation results for the case of switching directed interaction topology are presented in Section 3.5.3.

\subsubsection{Fixed Strongly-connected Directed Graph Communication Topology}

Let the communication topology be as shown in Fig. 3.1, where the weights are all set to be 1.

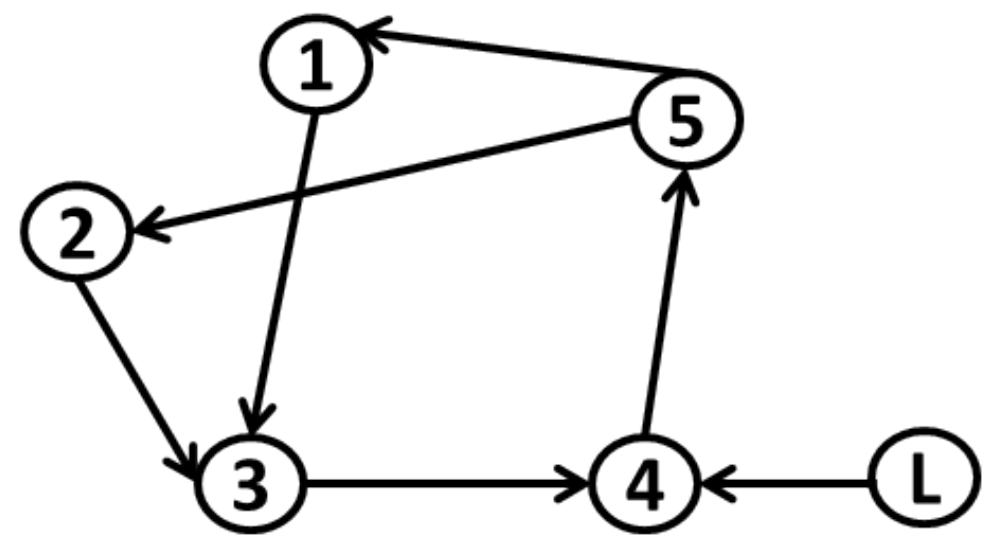

Figure 3.1: A fixed digraph communication topology

We will use an RBFNN with 27 neurons for each agent in our simulation. The configurations of RBFNN are the same for all five agents. The parameters in the RBFNN weight tuning law are selected as $\chi_{i}=1$ and $W_{\max i}=20000, i=1,2, \cdots, 5$. And the initial neural network weights are all set to be 0 . The RBFNN activation functions are selected to have following form

$$
\varphi_{i j}\left(x_{i}\right)=30 e^{-\frac{\left\|x_{i}-c_{j}\right\|^{2}}{1000}}, i=1,2, \cdots, 5 ; j=1,2, \cdots, 27
$$

where, $c_{(j)}$ are the centers of the RBFNN activation functions, which are distributed evenly in the cube $[-1,1] \times[-1,1] \times[-1,1]$.

The initial states of the five agents are $x_{1}=\left[\begin{array}{lll}15 & 1 & 10\end{array}\right]^{\mathrm{T}}, x_{2}=\left[\begin{array}{lll}2 & 20 & 2\end{array}\right]^{\mathrm{T}}, x_{3}=\left[\begin{array}{llll}3 & 12.5 & 1.5\end{array}\right]^{\mathrm{T}}, x_{4}=\left[\begin{array}{lll}4 & 32 & 3\end{array}\right]^{\mathrm{T}}$, and $x_{5}=\left[\begin{array}{lll}-5 & 40 & 4\end{array}\right]^{\mathrm{T}}$. The initial state of the leader agent is $x_{0}=\left[\begin{array}{lll}10 & 20 & 8\end{array}\right]^{\mathrm{T}}$.

Case 1 Using the control protocol defined by (3.3) and (3.6) with the aforementioned parameters, we simulate the multi-agent system from $t=0.0$ to $t=14.0 \mathrm{~s}$ with $c=100$. The synchronized motion phase plane plot of all five agents and the leader agent is shown in Fig. 3.2, where the blue line represents the leader agent and the red lines represent the five follower agents. The "X" symbols stand for the initial states, and the "+" 
symbols mean the states at the end of the simulation. As time evolves, the states of all the five follower agents are synchronized to the state of the leader agent very well. This can also be seen from the tracking error plot of position, velocity and acceleration-see Fig. 3.3. Basically, as time evolves, all these tracking errors are approximately zero. Fig. 3.4 plots the unknown nonlinear function $f_{i}\left(x_{i}\right)$ as well as the neural network approximation $\hat{f}_{i}\left(x_{i}\right), i=1,2, \cdots, 5$. In Fig. 3.4, the black lines represent $f_{i}\left(x_{i}\right)$ and the red lines are $\hat{f}_{i}\left(x_{i}\right)$. As we can see, the RBFNN can approximate the unknown nonlinear function $f_{i}\left(x_{i}\right)$ very well. Based on Fig. 3.4, the uncertainty compensation capability of the proposed RBFNN is illustrated.

Case 2 This case is used to illustrate the impact of the control parameter $c$. With the control protocol defined by (3.3) and (3.6), the multi-agent system is simulated from $t=0.0$ to $t=10.0 \mathrm{~s}$ with 1) $c=20$; 2) $c=30$; 3) $c=60$; 4) $c=100$. The tracking error plots are shown in Fig. 3.5, Fig. 3.6, Fig. 3.7 and Fig. 3.8, respectively. As we can see, the control parameter c has significant impact on the synchronization control performance. The ultimate bound of synchronization error can be reduced by increasing the value of control parameter $c$.

Case 3 The RBFNN uncertainty compensation ability has already been illustrated by Fig. 3.4 in Case 1. This case is used to further investigate the impact of the RBFNN upon the synchronization control performance. The multi-agent system is simulated from $t=0.0$ to $t=8.0 \mathrm{~s}$ in following three different cases: 1$)$ use the control rule specified by (3.3) and (3.6) with control parameter $c=60$; 2) use the control rule $u_{i}=$ cr $_{i}$ with control parameter $c=60$; 3) use the control rule $u_{i}=c_{i}$ with control parameter $c=500$. It is easy to see that the control rule $u_{i}=c r_{i}$ just removes the RBFNN from the proposed control protocol. The synchronized motion phase plane plots are shown in Fig. 3.9, Fig. 3.10 and Fig. 3.11, respectively. The tracking error plots are shown in Fig. 3.12, Fig. 3.13 and Fig. 3.14, respectively. By comparing Fig. 3.9, Fig. 3.10, Fig. 3.12 and Fig. 3.13, we know that the employment of the RBFNN can significantly reduce the ultimate bound of the synchronization error. By comparing Fig. 3.9, Fig. 3.11, Fig. 3.12 and Fig. 3.14, we know that, without the RBFNN, the control protocol will require a much larger control gain c to achieve the same ultimate synchronization error bound. This illustrates the benefits to employ the RBFNN in our control protocols. 


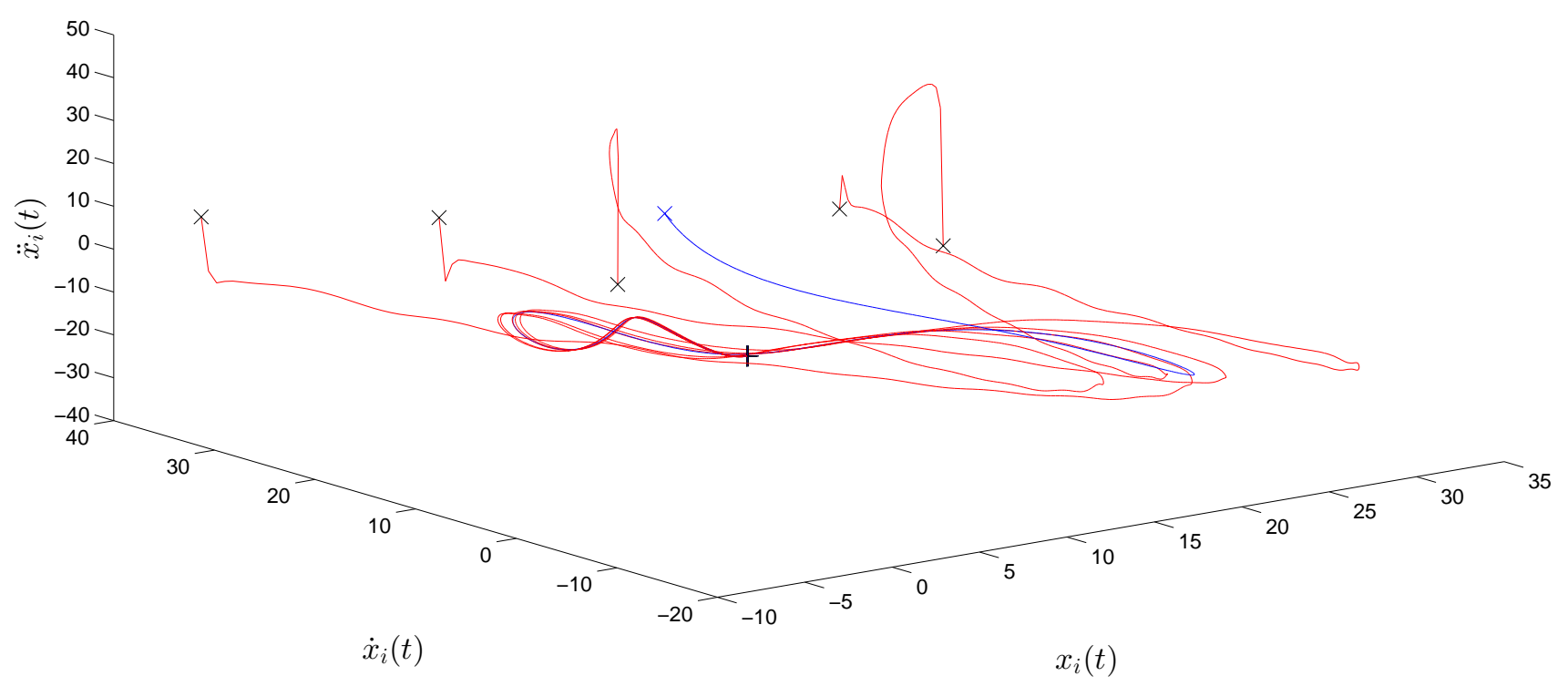

Figure 3.2: Phase plot, fixed digraph topology, Case 1, c=100

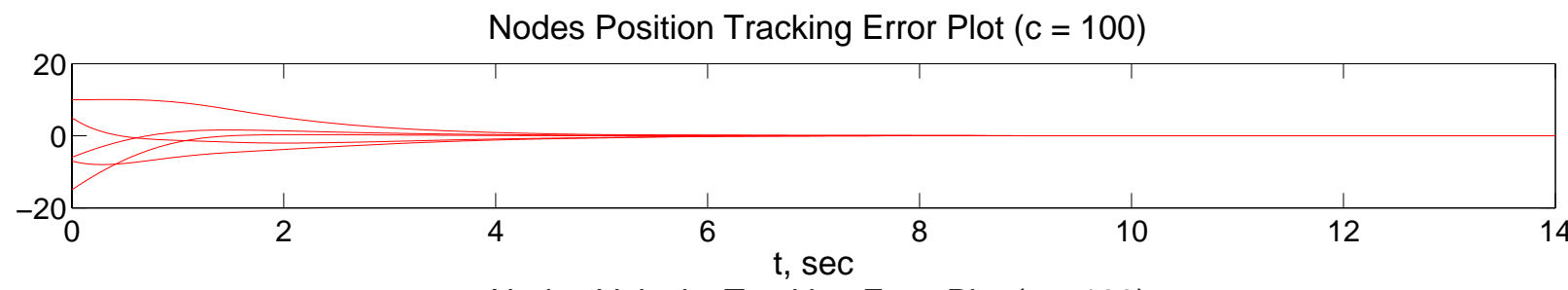

Nodes Velocity Tracking Error Plot $(c=100)$

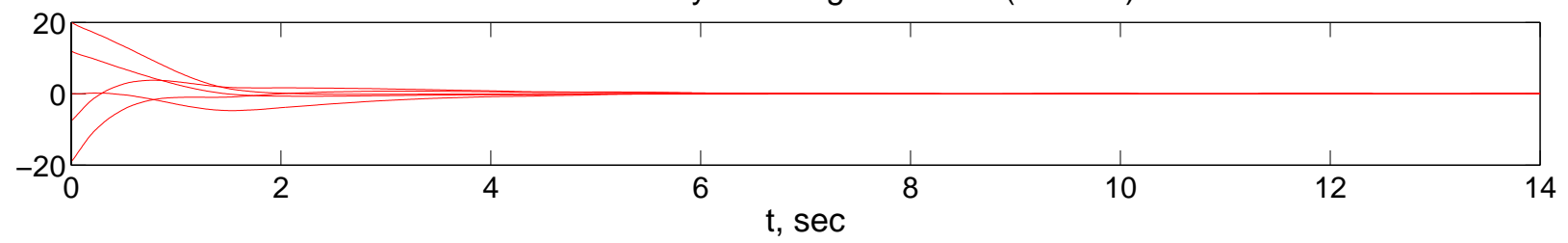

Nodes Acceleration Tracking Error Plot $(c=100)$

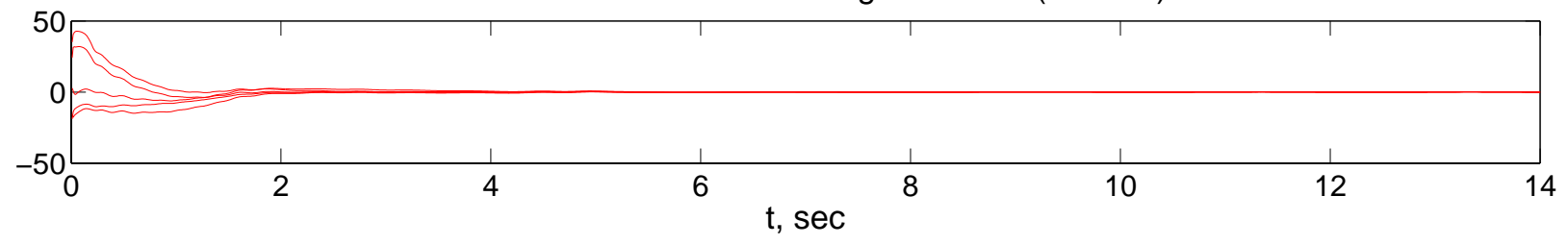

Figure 3.3: Tracking error plot, fixed digraph topology, Case 1, c=100 


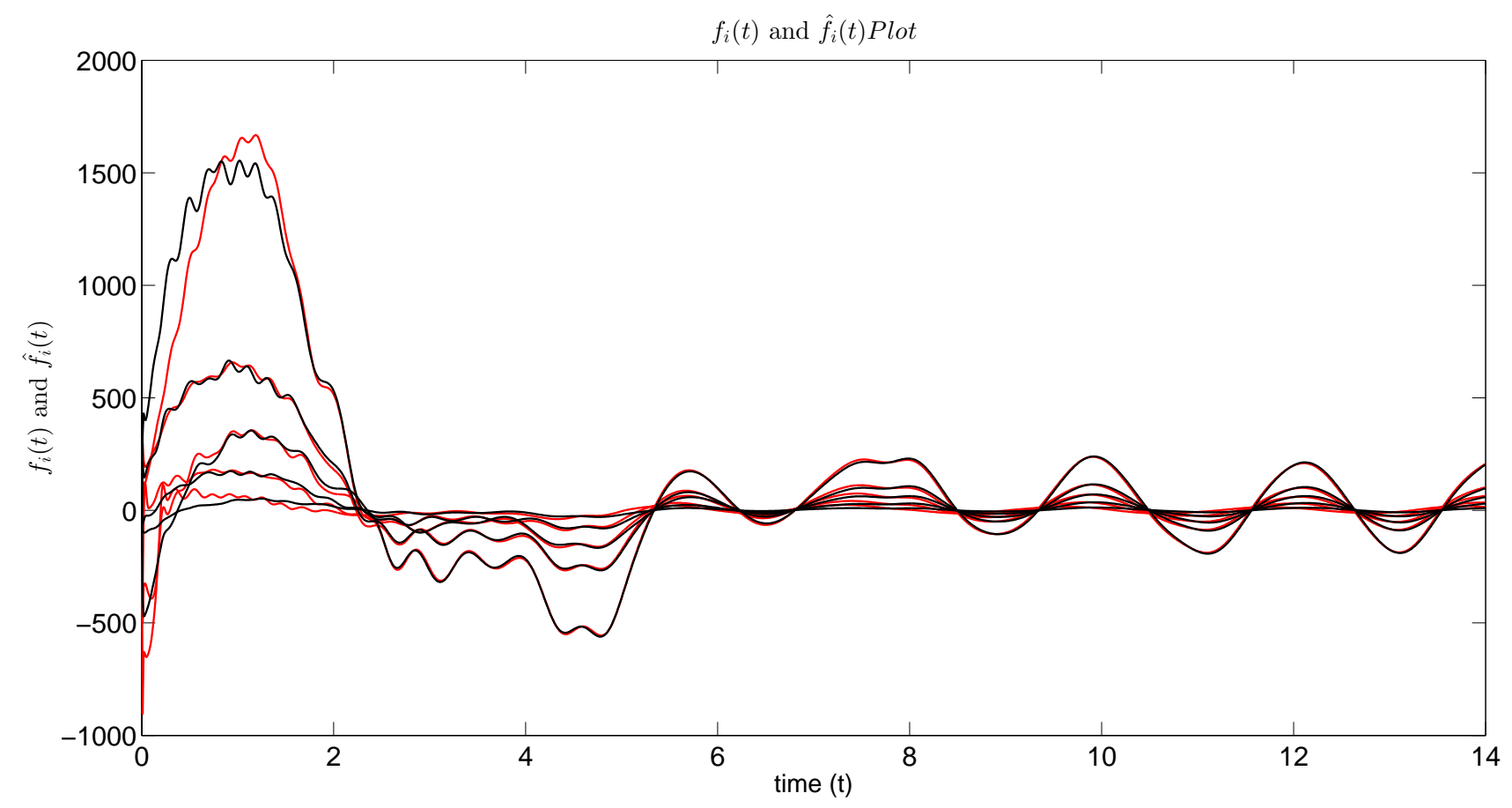

Figure 3.4: RBFNN approximation, fixed digraph topology, Case 1, $c=100$

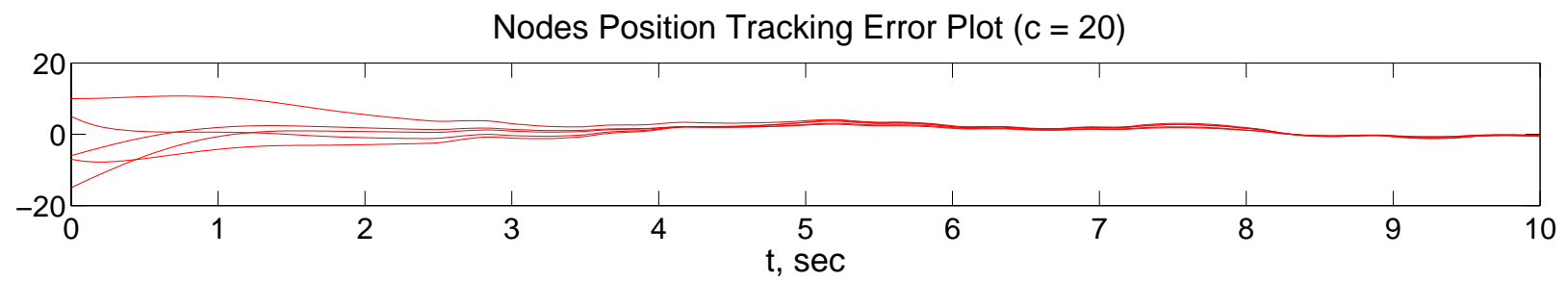

Nodes Velocity Tracking Error Plot $(c=20)$

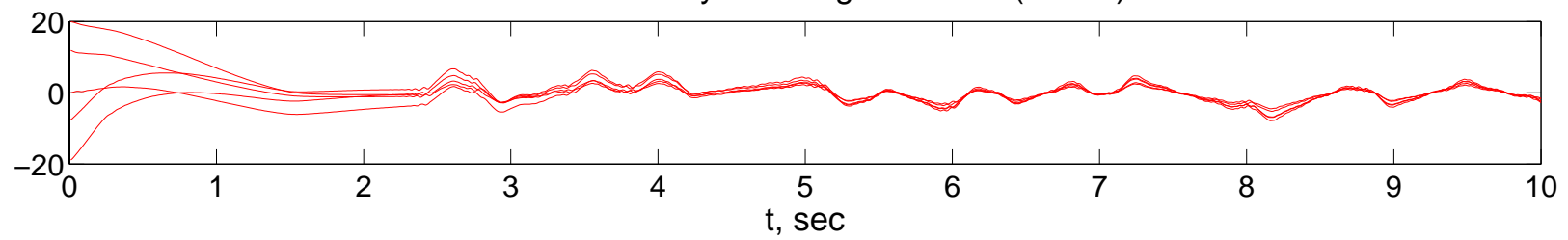

Nodes Acceleration Tracking Error Plot $(c=20)$

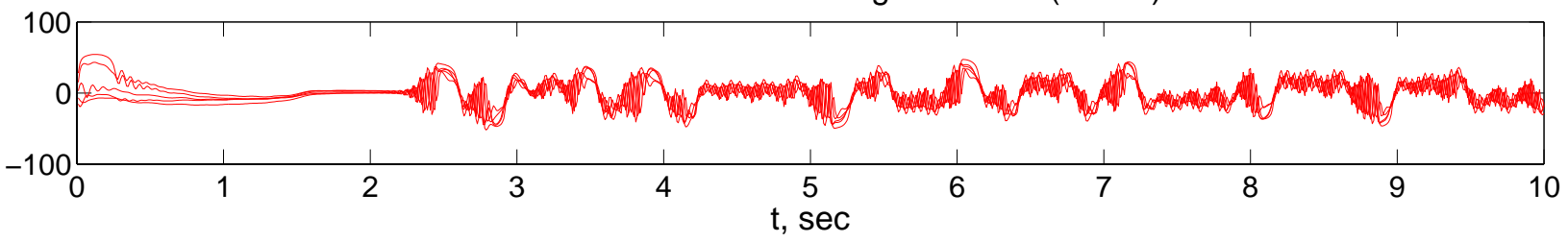

Figure 3.5: Tracking error plot, fixed digraph topology, Case 2, $\mathrm{c}=20$ 
Nodes Position Tracking Error Plot $(c=30)$
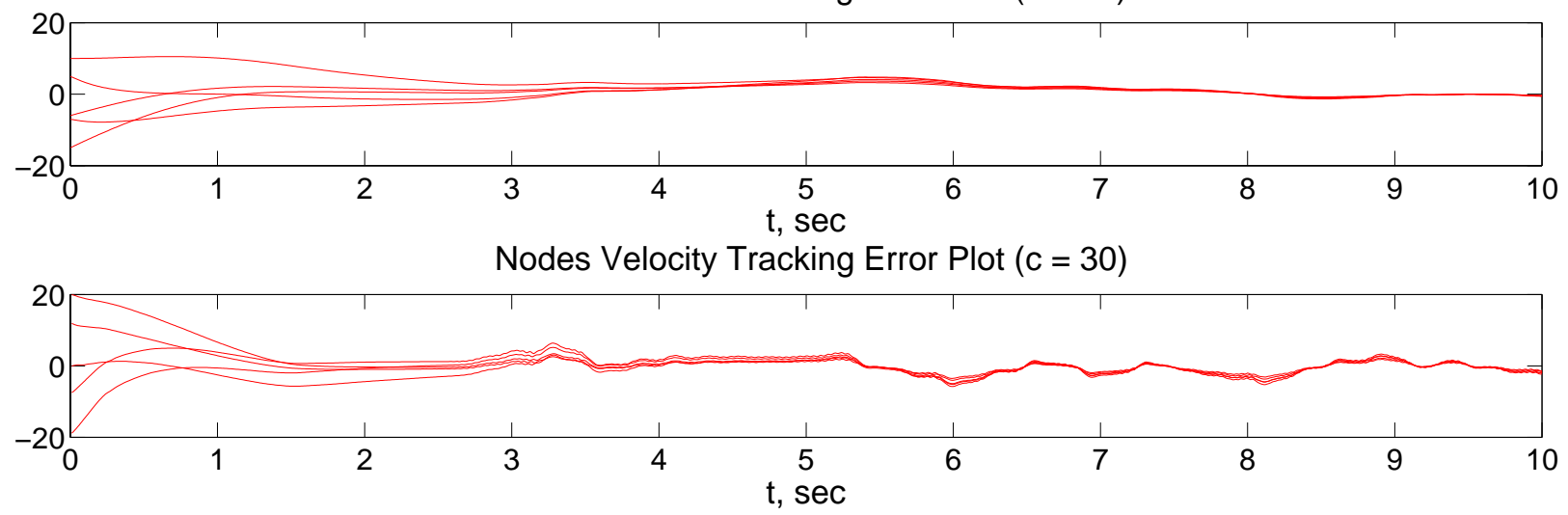

Nodes Acceleration Tracking Error Plot $(c=30)$

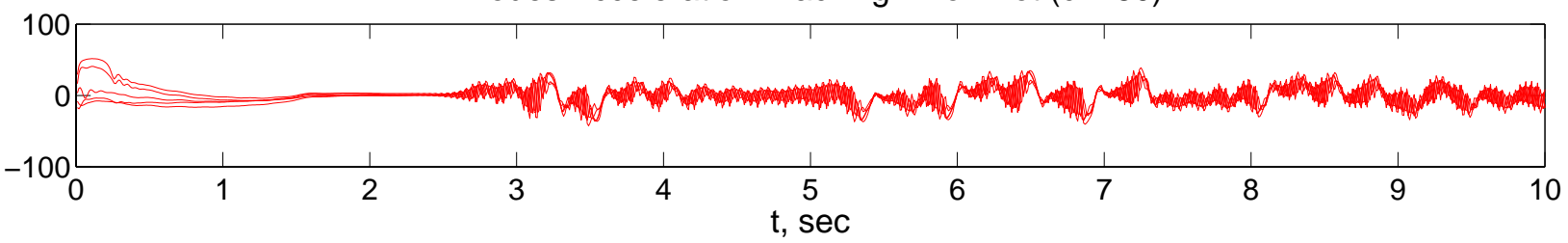

Figure 3.6: Tracking error plot, fixed digraph topology, Case 2, $\mathrm{c}=30$

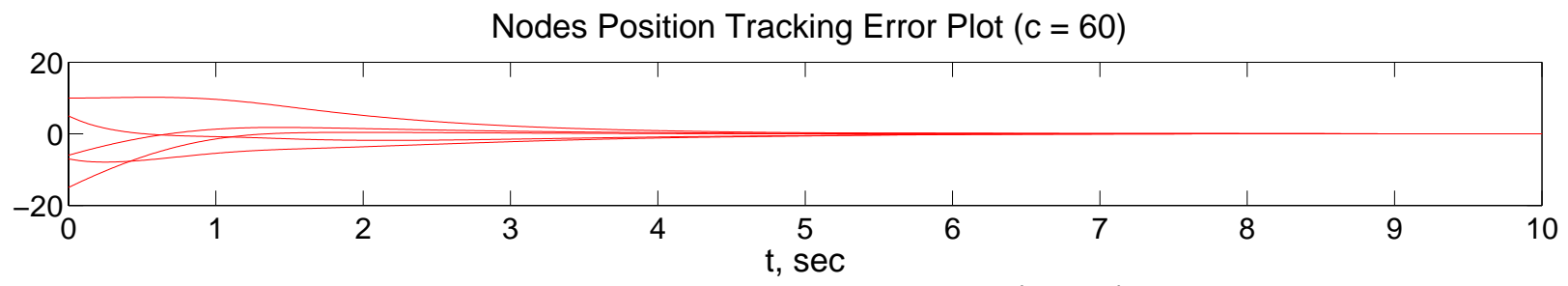

Nodes Velocity Tracking Error Plot $(c=60)$

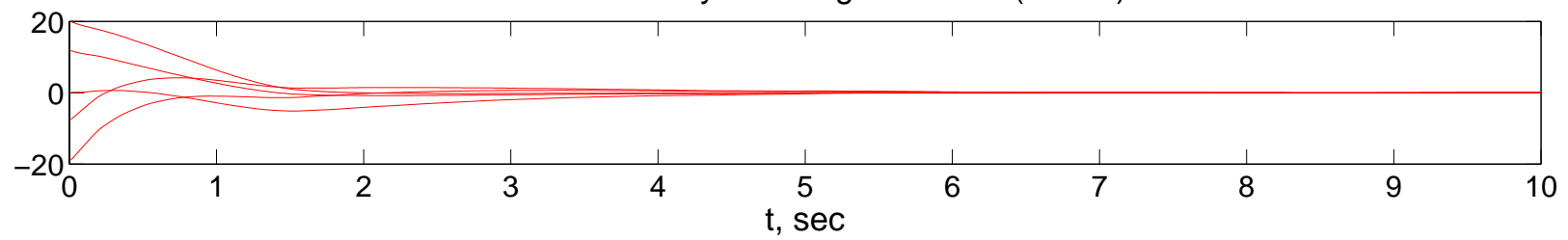

Nodes Acceleration Tracking Error Plot $(c=60)$

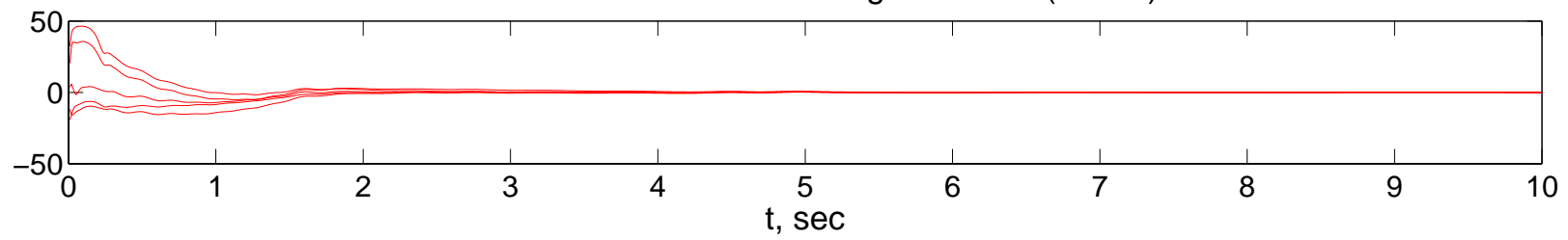

Figure 3.7: Tracking error plot, fixed digraph topology, Case 2, $\mathrm{c}=60$ 
Nodes Position Tracking Error Plot $(c=100)$

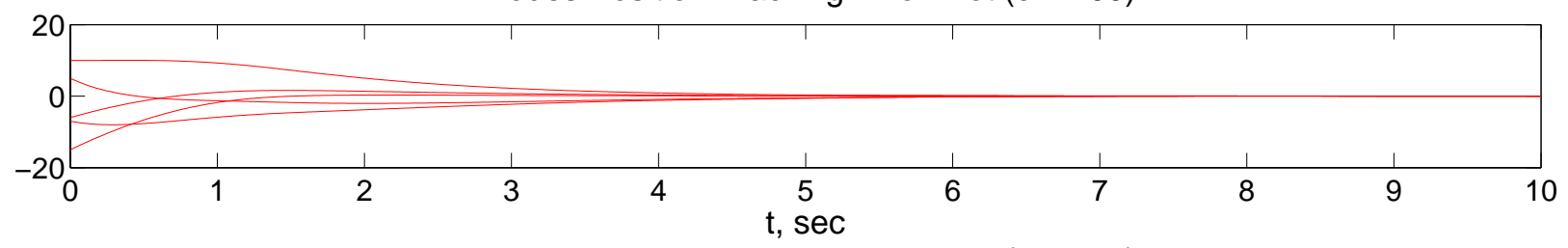

Nodes Velocity Tracking Error Plot $(c=100)$

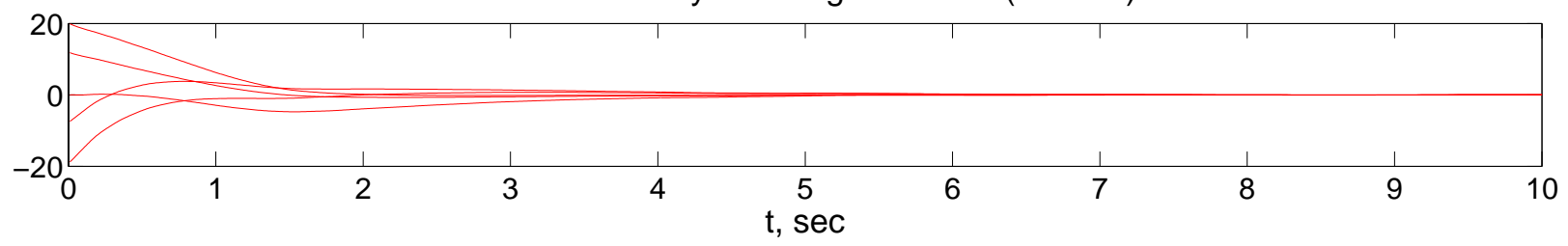

Nodes Acceleration Tracking Error Plot $(c=100)$

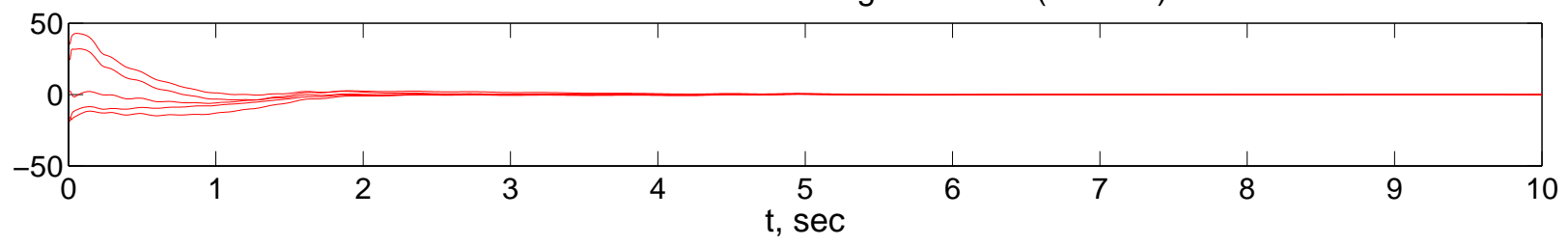

Figure 3.8: Tracking error plot, fixed digraph topology, Case 2, c=100

Nodes Synchronized Motion Phase Plane Plot $(c=60)$

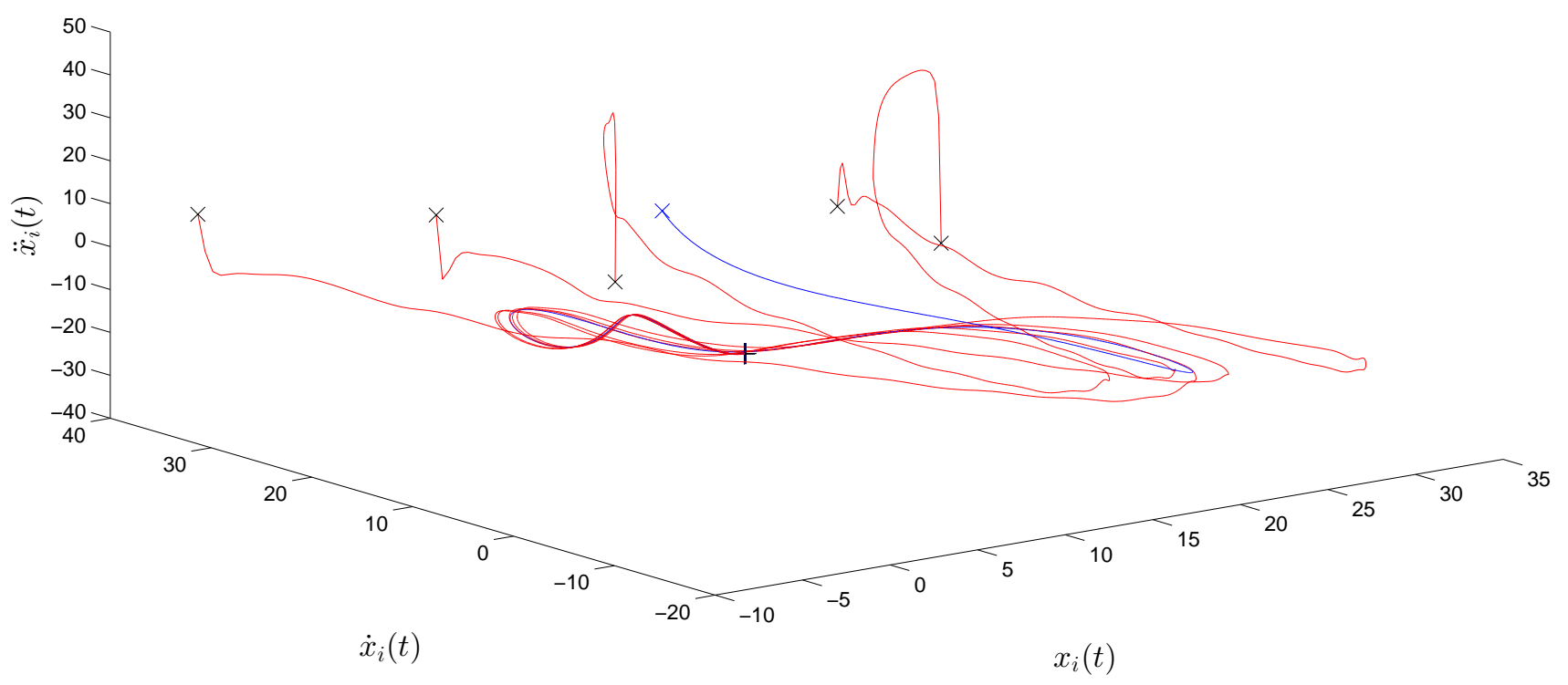

Figure 3.9: Phase plot, fixed digraph topology, Case 3, c=60, with RBFNN 


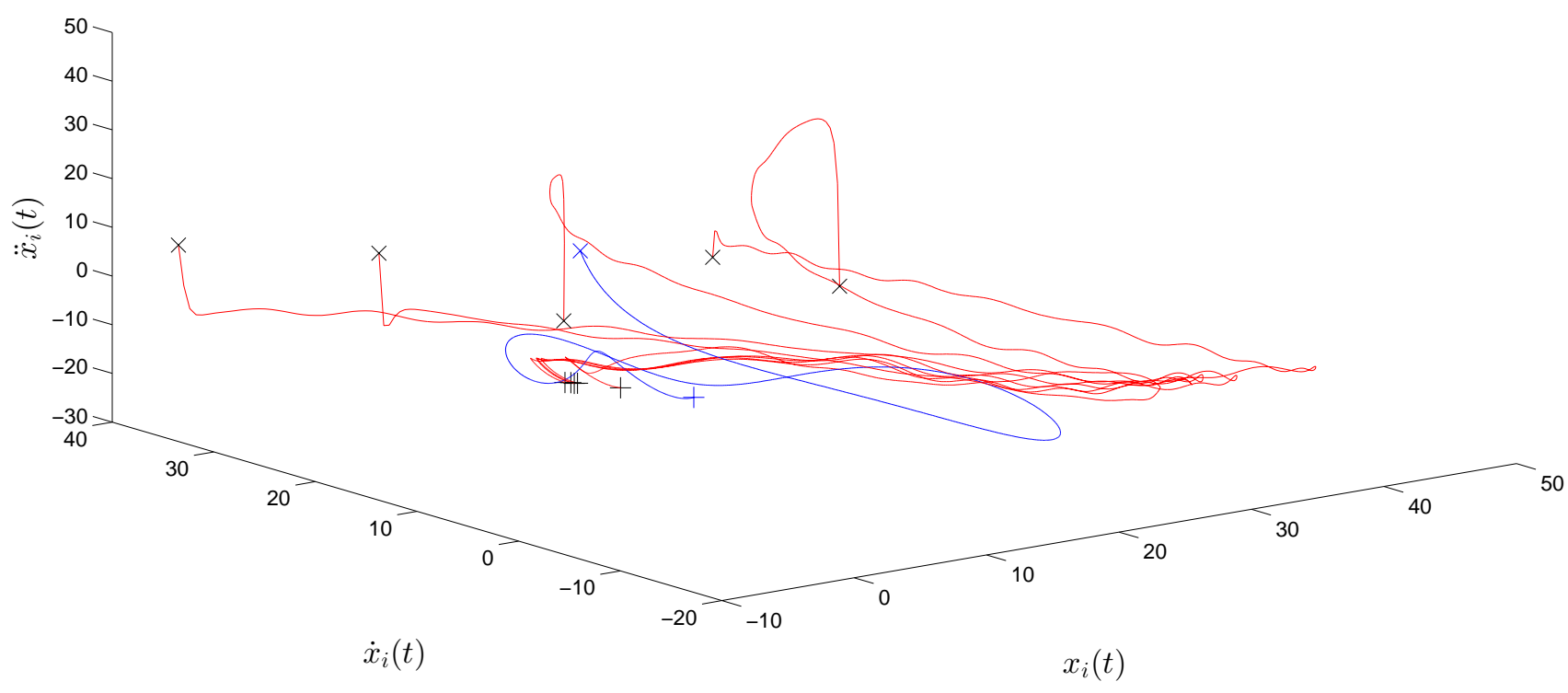

Figure 3.10: Phase plot, fixed digraph topology, Case 3, $c=60$, without RBFNN

Nodes Synchronized Motion Phase Plane Plot (No RBFNN) $(c=200)$

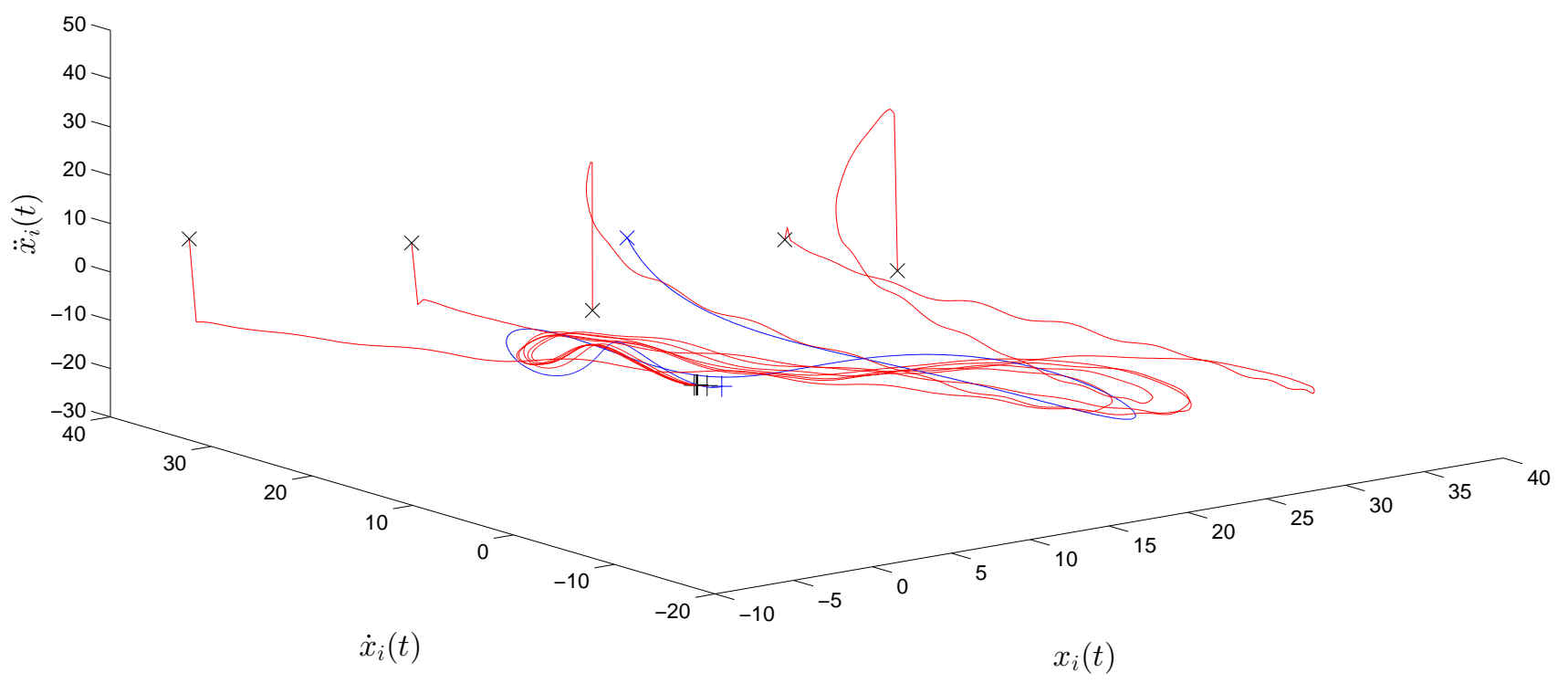

Figure 3.11: Phase plot, fixed digraph topology, Case 3, c=200, without RBFNN 
Nodes Position Tracking Error Plot $(c=60)$
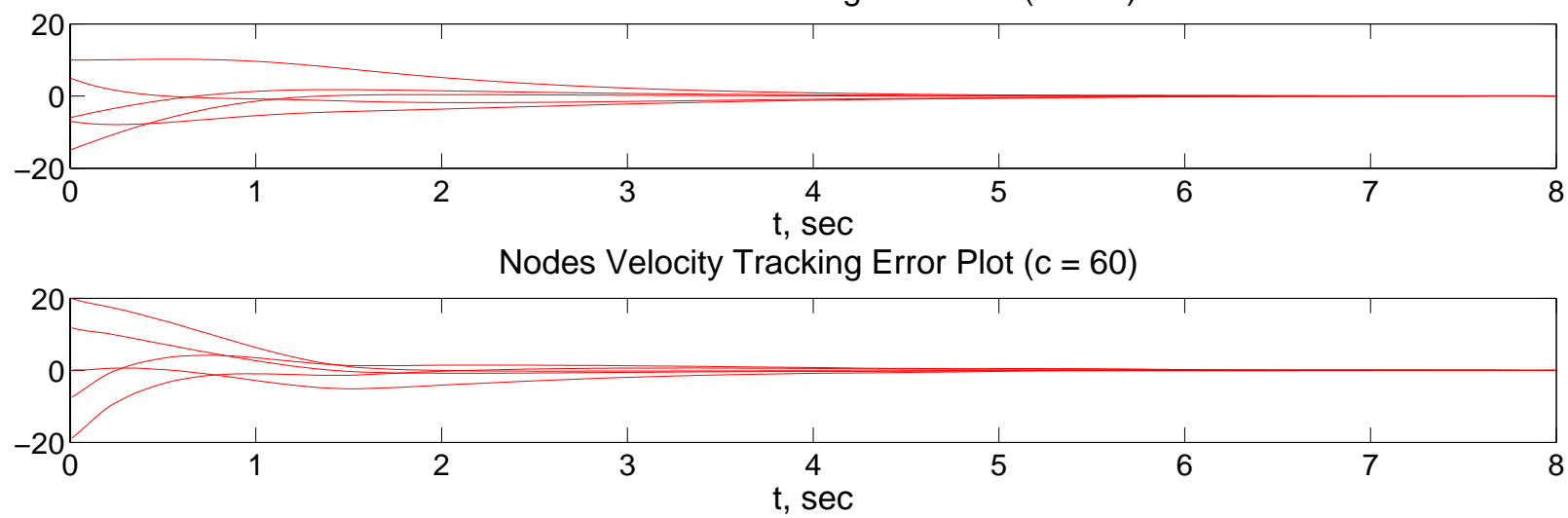

Nodes Acceleration Tracking Error Plot $(c=60)$

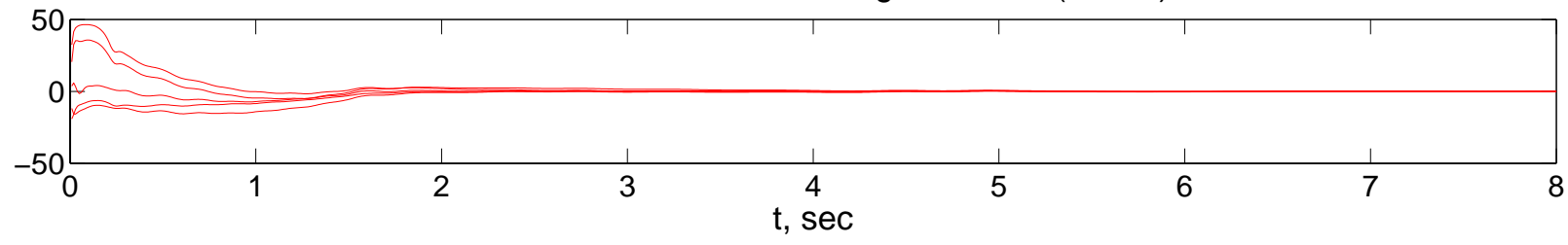

Figure 3.12: Tracking error plot, fixed digraph topology, Case 3, $c=60$, with RBFNN

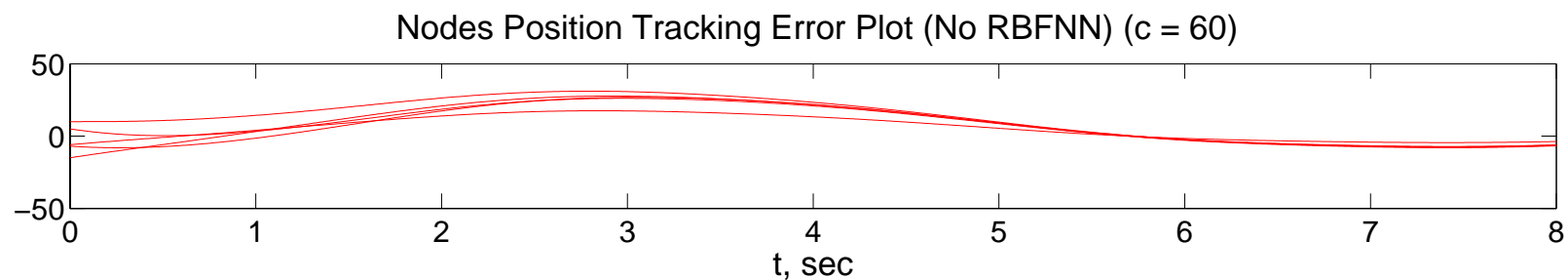

Nodes Velocity Tracking Error Plot (No RBFNN) $(c=60)$

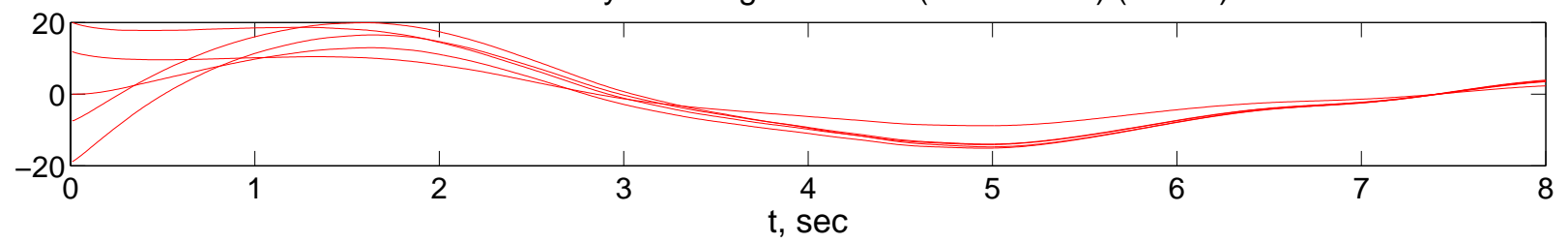

Nodes Acceleration Tracking Error Plot (No RBFNN) $(c=60)$

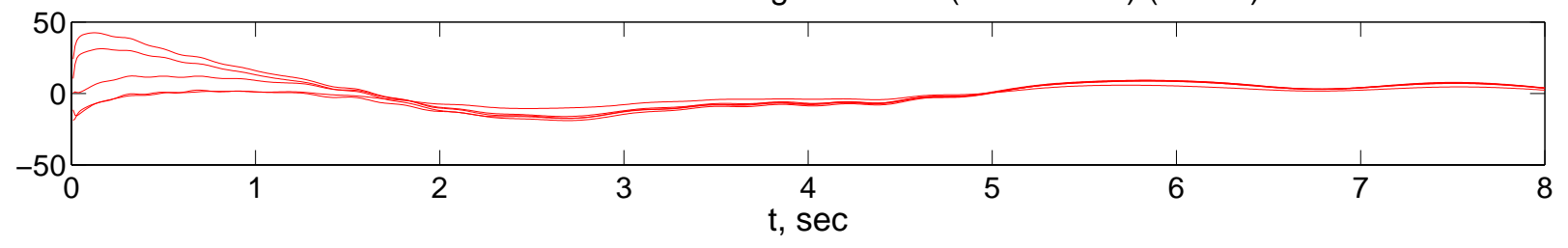

Figure 3.13: Tracking error plot, fixed digraph topology, Case $3, c=60$, without RBFNN 
Nodes Position Tracking Error Plot (No RBFNN) (c = 200)
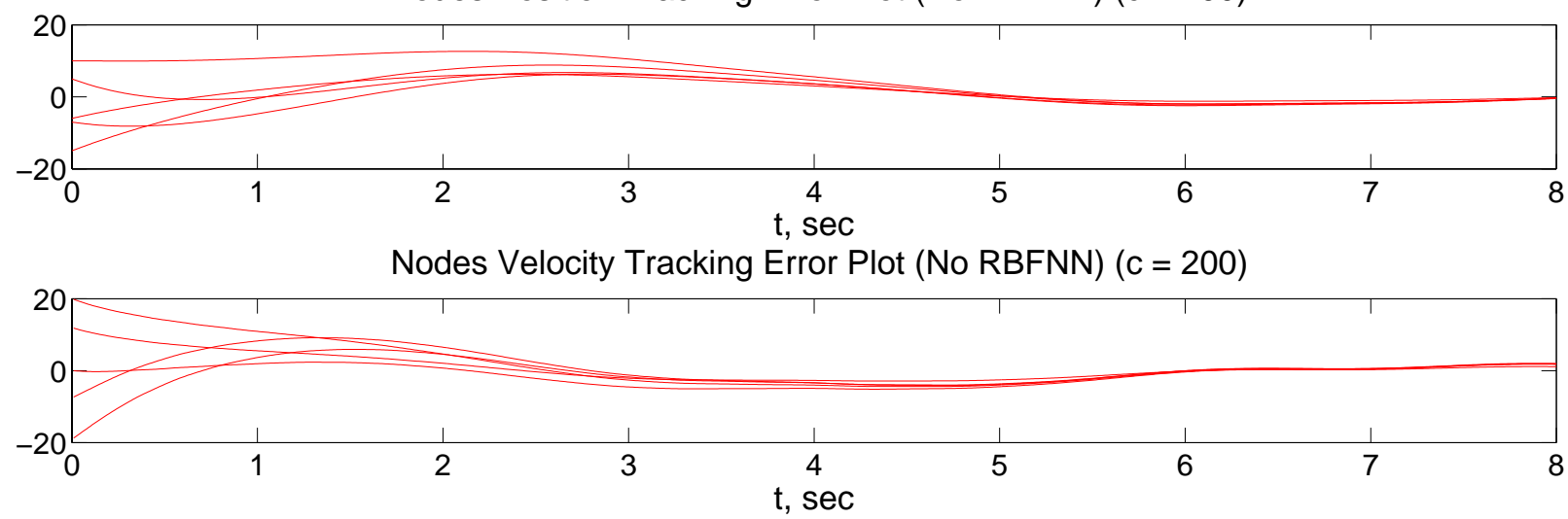

Nodes Acceleration Tracking Error Plot (No RBFNN) (c=200)

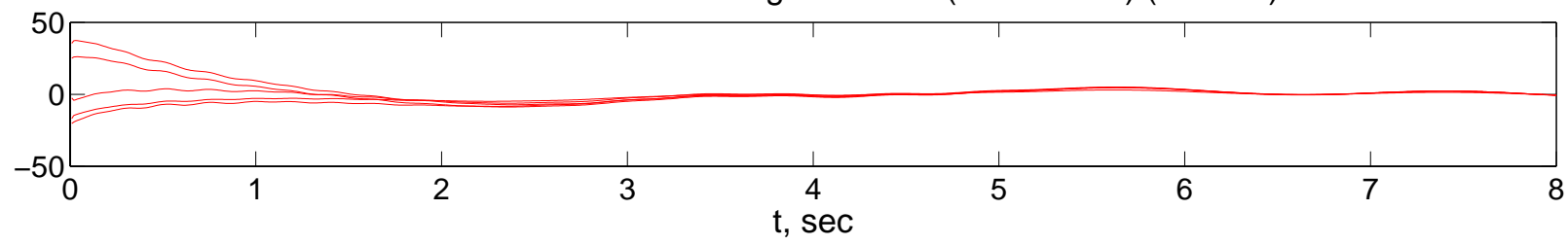

Figure 3.14: Tracking error plot, fixed digraph topology, Case 3, $c=200$, without RBFNN

\subsubsection{Switching Connected Undirected Graph Communication Topology}

Let the switching connected undirected communication topology be as shown in Fig. 3.15. The weight of each edge is also shown in the figure. The set $\left\{G_{a}, G_{b}, G_{c}, G_{d}, G_{e}, G_{f}\right\}$ represents the six different network topologies of the multi-agent system. The multi-agent system starts at topology $G_{a}$ and switches every $T=0.1 s$ to the next topology. Note that $G_{a}$ is set to be the next topology of $G_{f}$. Namely, the network topology is switching according to the following rule: $G_{a} \rightarrow G_{b} \rightarrow G_{c} \rightarrow G_{d} \rightarrow G_{e} \rightarrow G_{f} \rightarrow G_{a} \rightarrow G_{b} \cdots$.

We will again use an RBFNN with 27 neurons for each agent in our simulation. The configurations of RBFNN are the same for all five agents. The control parameters are selected as $c=60, \chi_{i}=1$ and $W_{\max i}=20000, i=1,2, \cdots, 5$. And the initial neural network weights are all set to be 0 . The RBFNN activation functions are selected to have following form

$$
\varphi_{i j}\left(x_{i}\right)=30 e^{-\frac{\left\|x_{i}-c_{j}\right\|^{2}}{1000}}, j=1,2, \cdots, 27 ; i=1,2, \cdots, 5
$$

where, $\left.c_{(} j\right)$ are the centers of the RBFNN activation functions, which are distributed evenly in the cube $[-1,1] \times[-1,1] \times[-1,1]$. 

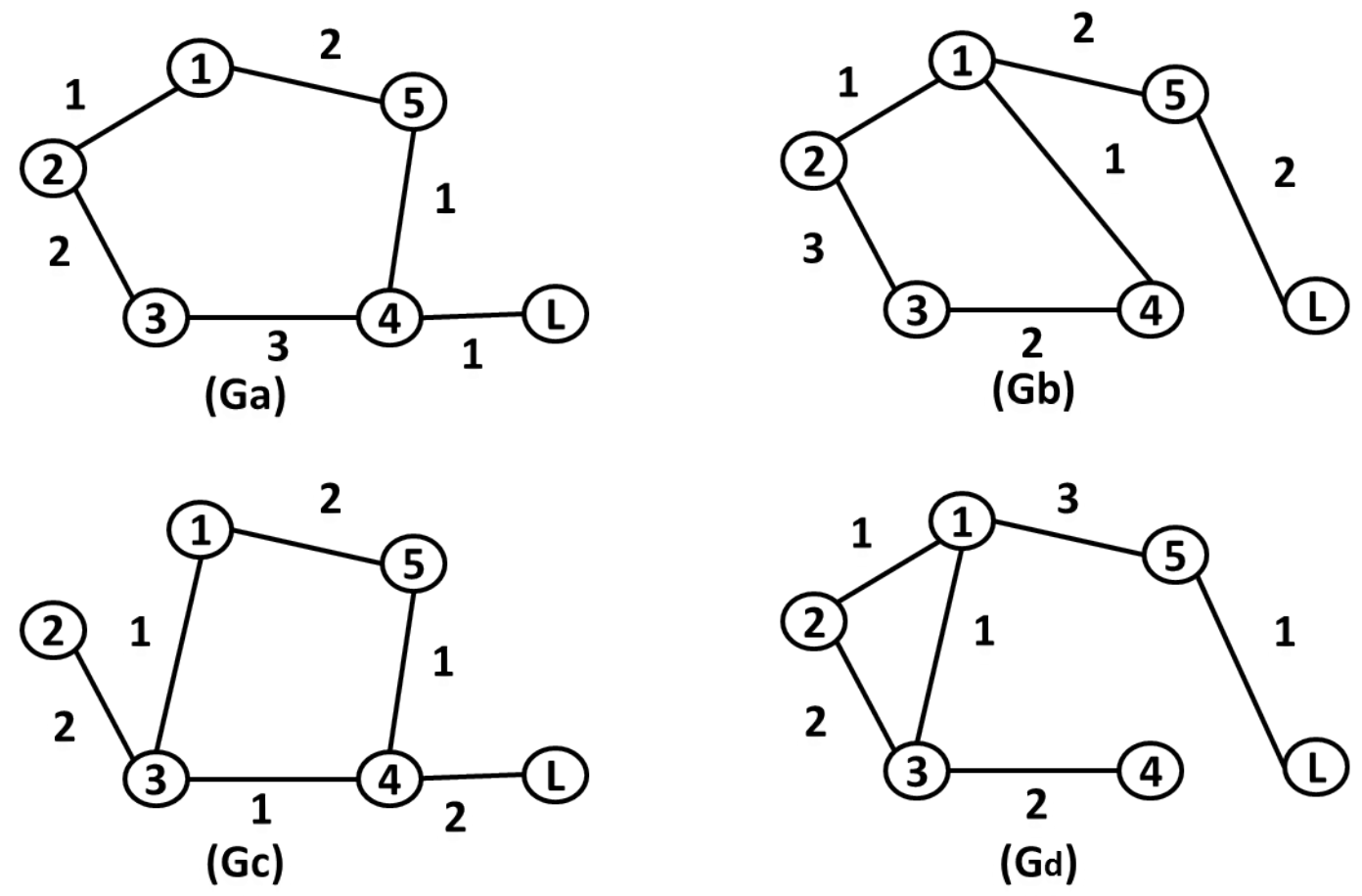

$(\mathrm{Gd})$

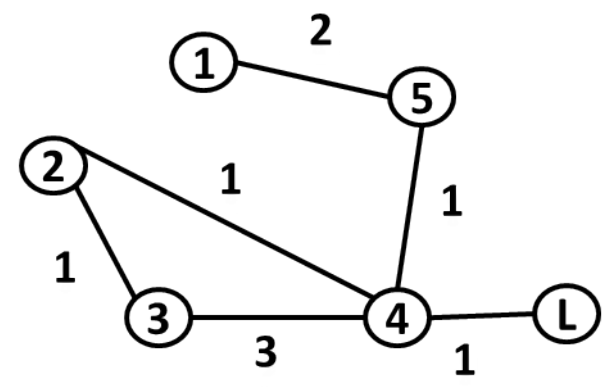

(Ge)

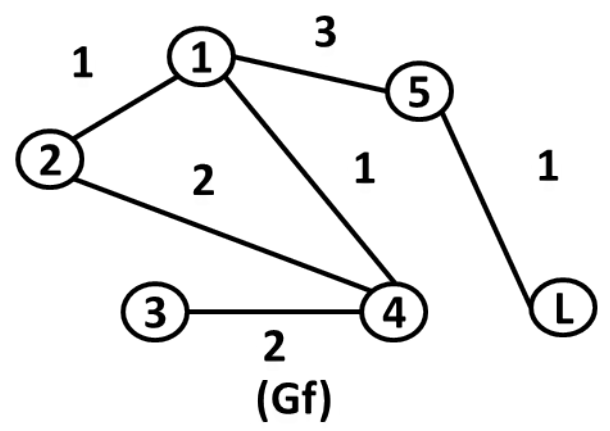

Figure 3.15: A switching connected undirected communication topology

The initial states of the five agents are $x_{1}=\left[\begin{array}{lll}10 & 25 & 3\end{array}\right]^{\mathrm{T}}, x_{2}=\left[\begin{array}{lll}-20 & 15 & 10\end{array}\right]^{\mathrm{T}}, x_{3}=\left[\begin{array}{lll}30 & 15 & -10\end{array}\right]^{\mathrm{T}}$, $x_{4}=\left[\begin{array}{lll}-4 & -20-12\end{array}\right]^{\mathrm{T}}$, and $x_{5}=\left[\begin{array}{lll}25 & 4 & 6\end{array}\right]^{\mathrm{T}}$. The initial state of the leader agent is $x_{0}=\left[\begin{array}{lll}15 & 10 & 6\end{array}\right]^{\mathrm{T}}$.

The multi-agent system is simulated from $t=0.0$ to $t=10.0 \mathrm{~s}$ with the aforementioned parameters. The synchronized motion phase plane plot of all five agents and the leader agent is shown in Fig. 3.16, where the blue line represents the leader agent and the red lines represent the five follower agents. The " $\times$ " symbols stand for the initial states, and the "+" symbols mean the states at the end of the simulation. As time evolves, the states of all the five follower agents are synchronized to the state of the leader agent very well. This can also be seen from the tracking error plot in Fig. 3.17. As we can see, as time evolves, all these tracking errors are approximately zero. Fig. 3.18 plots the unknown nonlinear function $f_{i}\left(x_{i}\right)$ as well as the 


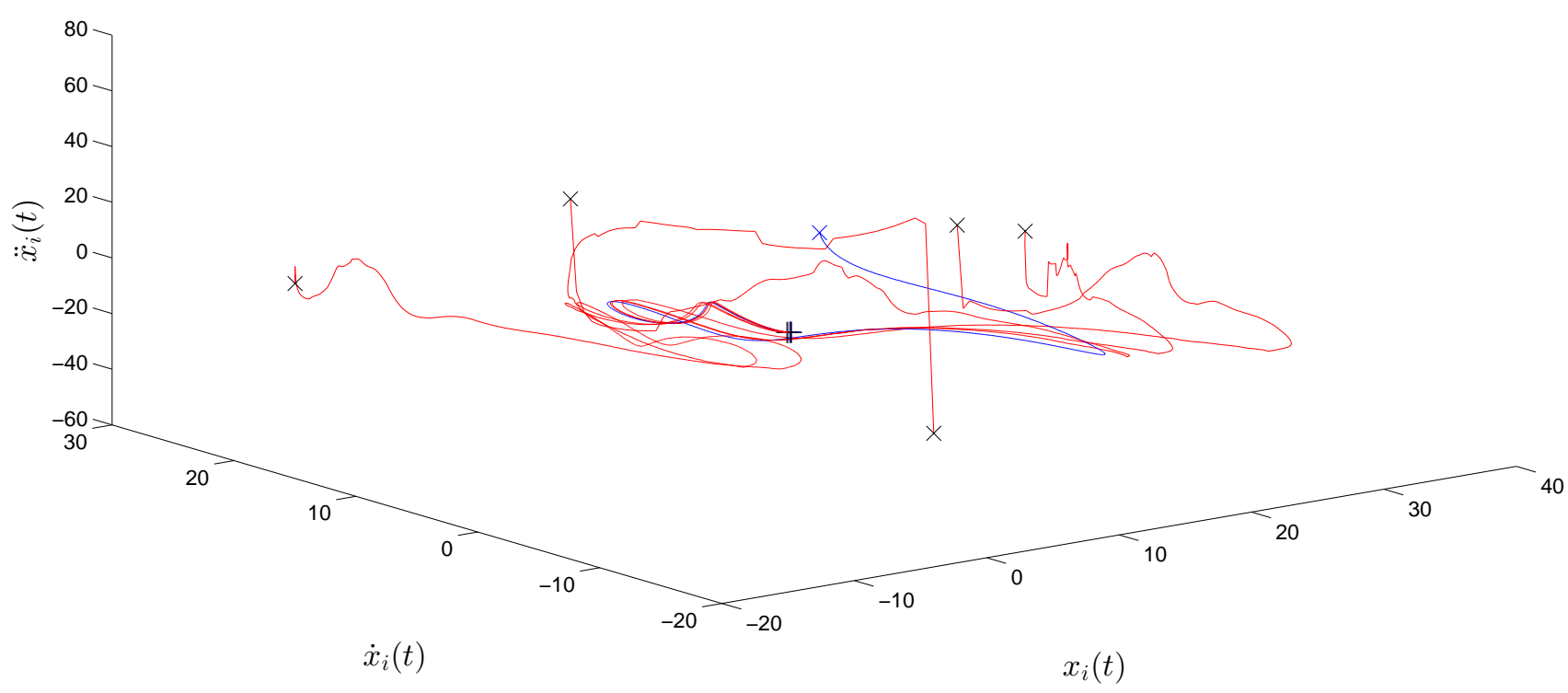

Figure 3.16: Phase plot, switching undirected graph topology, $\mathrm{c}=60$

corresponding neural network approximation $\hat{f}_{i}\left(x_{i}\right), i=1,2, \cdots, 5$, where the black lines represent $f_{i}\left(x_{i}\right)$ and the red lines are $\hat{f}_{i}\left(x_{i}\right)$. As shown in the figure, the RBFNN can captures the unknown uncertainties very well. Based on Fig. 3.18, the uncertainty compensation capability of the proposed RBFNN is illustrated. 
Nodes Position Tracking Error Plot $(c=60)$

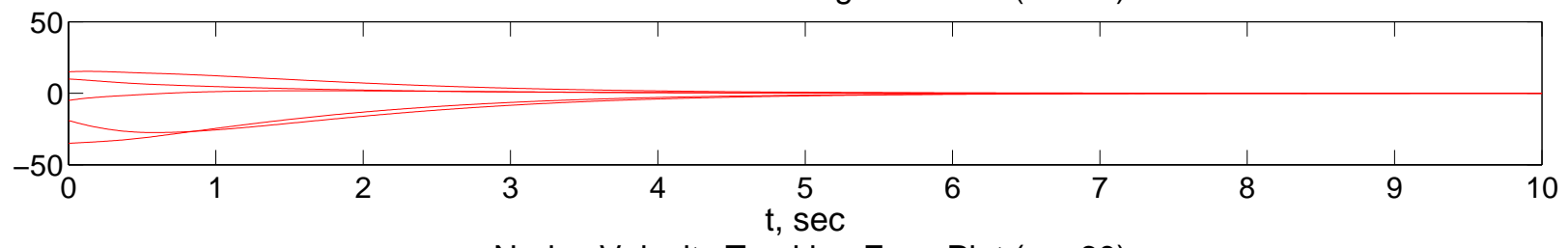

Nodes Velocity Tracking Error Plot $(c=60)$

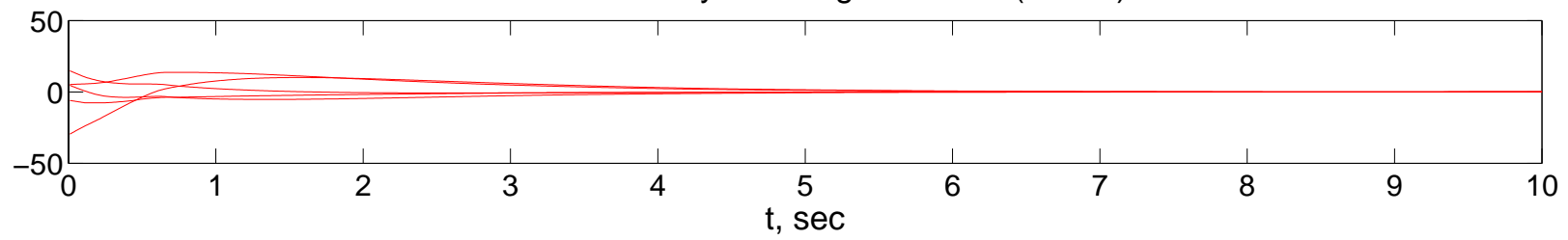

Nodes Acceleration Tracking Error Plot $(c=60)$

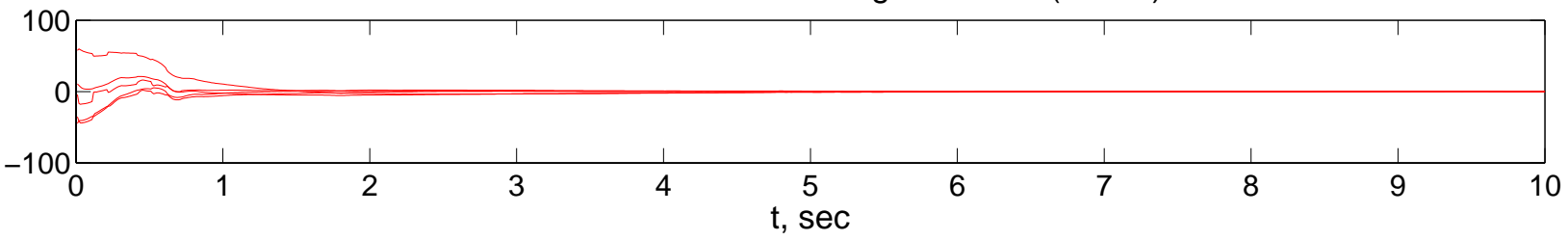

Figure 3.17: Tracking error plot, switching undirected graph topology, c=60

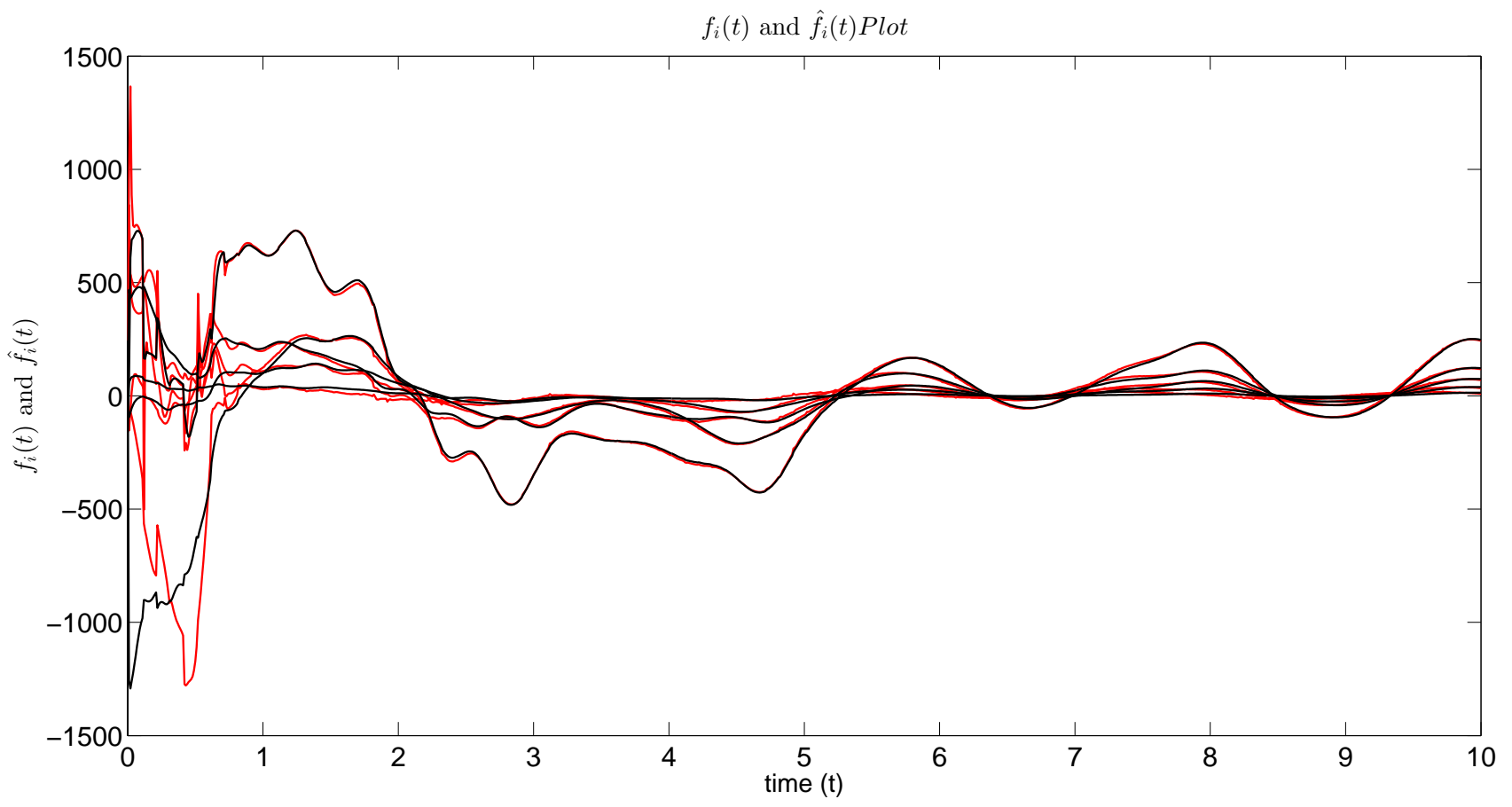

Figure 3.18: RBFNN approximation, switching undirected graph topology, $\mathrm{c}=60$ 


\subsubsection{Switching Strongly-connected Directed Graph Communication Topology}

In this subsection, simulation examples are provided to demonstrate the effectiveness of our proposed control protocols for the case of switching strongly-connected directed interaction topology.

\section{A. Example 1}

Let the communication topology be represented by a switching strongly-connected directed graph as shown in Fig. 3.19. The weight of each edge is also shown in the figure. The set $\left\{G_{1}, G_{2}, G_{3}, G_{4}, G_{5}, G_{6}\right\}$ represents the six different network topologies of the multi-agent system. It is easy to verify that all these six topologies are directed and strongly connected. The multi-agent system starts at topology $G_{1}$ and switches every $T=0.2$ to the next topology, with $G_{6}$ followed by $G_{1}$. Namely, the network topology is switching according to the following rule: $G_{1} \rightarrow G_{2} \rightarrow G_{3} \rightarrow G_{4} \rightarrow G_{5} \rightarrow G_{6} \rightarrow G_{1} \rightarrow G_{2} \cdots$.

We will again use an RBFNN with 27 neurons for each agent in our simulation. The control parameters are selected as $c=60, \chi_{i}=1$ and $W_{\max i}=20000, i=1,2, \cdots, 5$. The initial neural network weights are all set to be 0 . The RBFNN activation functions are selected to have the following form

$$
\varphi_{i j}\left(x_{i}\right)=30 e^{-\frac{\left\|x_{i}-c_{j}\right\|^{2}}{1000}}, j=1,2, \cdots, 27, i=1,2, \cdots, 5
$$

where, $c_{j}$ are the centers of the RBFNN activation functions, which are distributed evenly in the cube $[-1,1] \times[-1,1] \times[-1,1]$

The initial states of the five follower agents are $x_{1}^{(0)}=\left[\begin{array}{lll}10 & 25 & 3\end{array}\right]^{\mathrm{T}}, x_{2}^{(0)}=\left[\begin{array}{lll}-2 & 15 & 10\end{array}\right]^{\mathrm{T}}, x_{3}^{(0)}=\left[\begin{array}{ll}30 & 15-10\end{array}\right]^{\mathrm{T}}$, $x_{4}^{(0)}=\left[\begin{array}{lll}-4 & -20 & -12\end{array}\right]^{\mathrm{T}}$, and $x_{5}^{(0)}=\left[\begin{array}{lll}25 & 4 & 6\end{array}\right]^{\mathrm{T}}$. The initial state of the leader agent is $x_{0}^{(0)}=\left[\begin{array}{lll}15 & 10 & 6\end{array}\right]^{\mathrm{T}}$.

We simulate the multi-agent system using the control protocols defined by (3.27), (3.30) and (3.31) with the control gain parameter $c=60$. The state tracking error trajectories of all five follower agents are shown in Fig. 3.20. As we can see, the states of all the five follower agents synchronize to the state of the leader agent very well. The steady state synchronization errors are very close to zero.

The phase trajectories of the states, as is shown in Fig. 3.21, also help us to visualize the synchronization of the multi-agent system. In the figure, the blue dotted line denotes the phase trajectory of the leader agent and the red solid lines represent the phase trajectories of the five follower agents. The " $\square$ " symbols stand for the initial states, and the " $\triangle$ " symbols represent the states of the agents at the end of the simulation. As we can see, although starting from quite different initial states, the states of all the five follower agents synchronize to the state of the leader agent very well as time elapses. This can be easily observed by noticing 

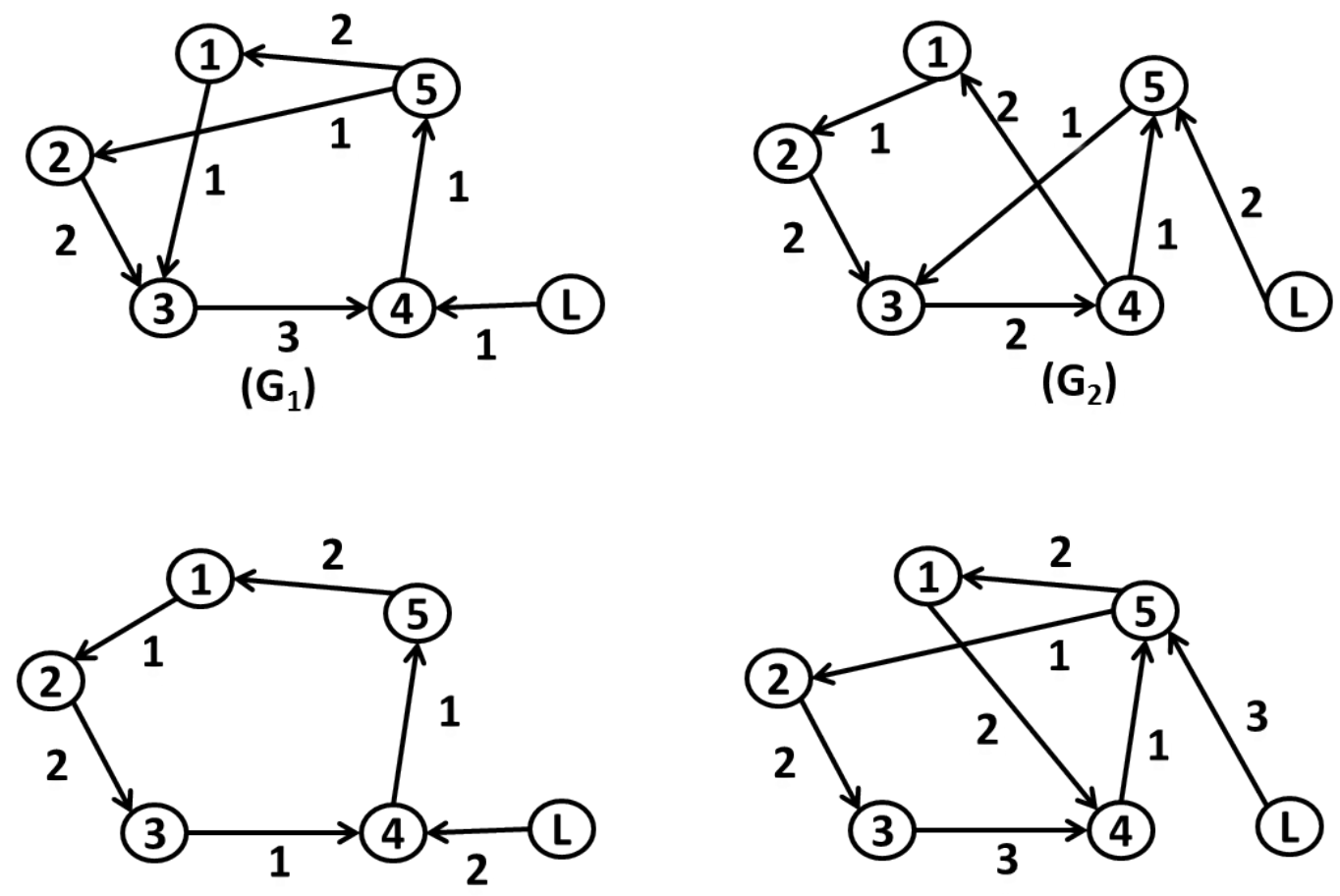

$\left(G_{3}\right)$

$\left(G_{4}\right)$

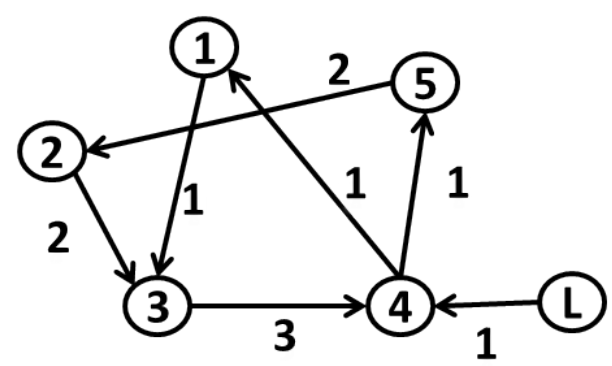

$\left(G_{5}\right)$

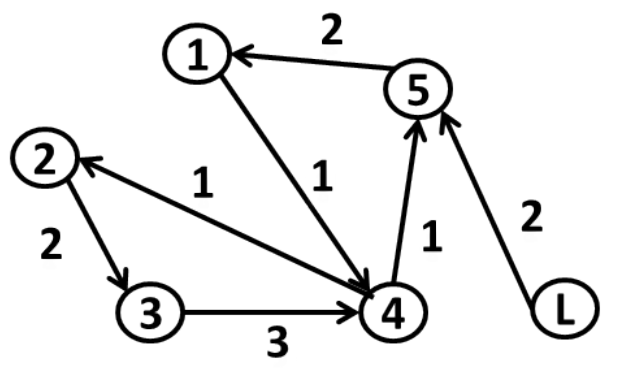

$\left(G_{6}\right)$

Figure 3.19: A switching strongly-connected directed graph communication topology

that all the six " $\triangle$ " symbols, which represent the states of the agents at the end of the simulation, are very close to each other.

As for the uncertainty compensation capability of the proposed RBFNN, it can be easily seen by plotting the unknown nonlinear function $f_{i}\left(x_{i}\right)$ as well as the corresponding neural network approximation $\widehat{f}_{i}\left(x_{i}\right)$, $i=1,2, \cdots, 5$, as is shown in Fig. 3.22. The blue dashed-dotted lines denote $f_{i}\left(x_{i}\right)$ and the black dashed lines represent $\widehat{f}_{i}\left(x_{i}\right)$. As seen in the figure, the RBFNNs approximate the unknown nonlinear functions $f_{i}\left(x_{i}\right)$ very well. 

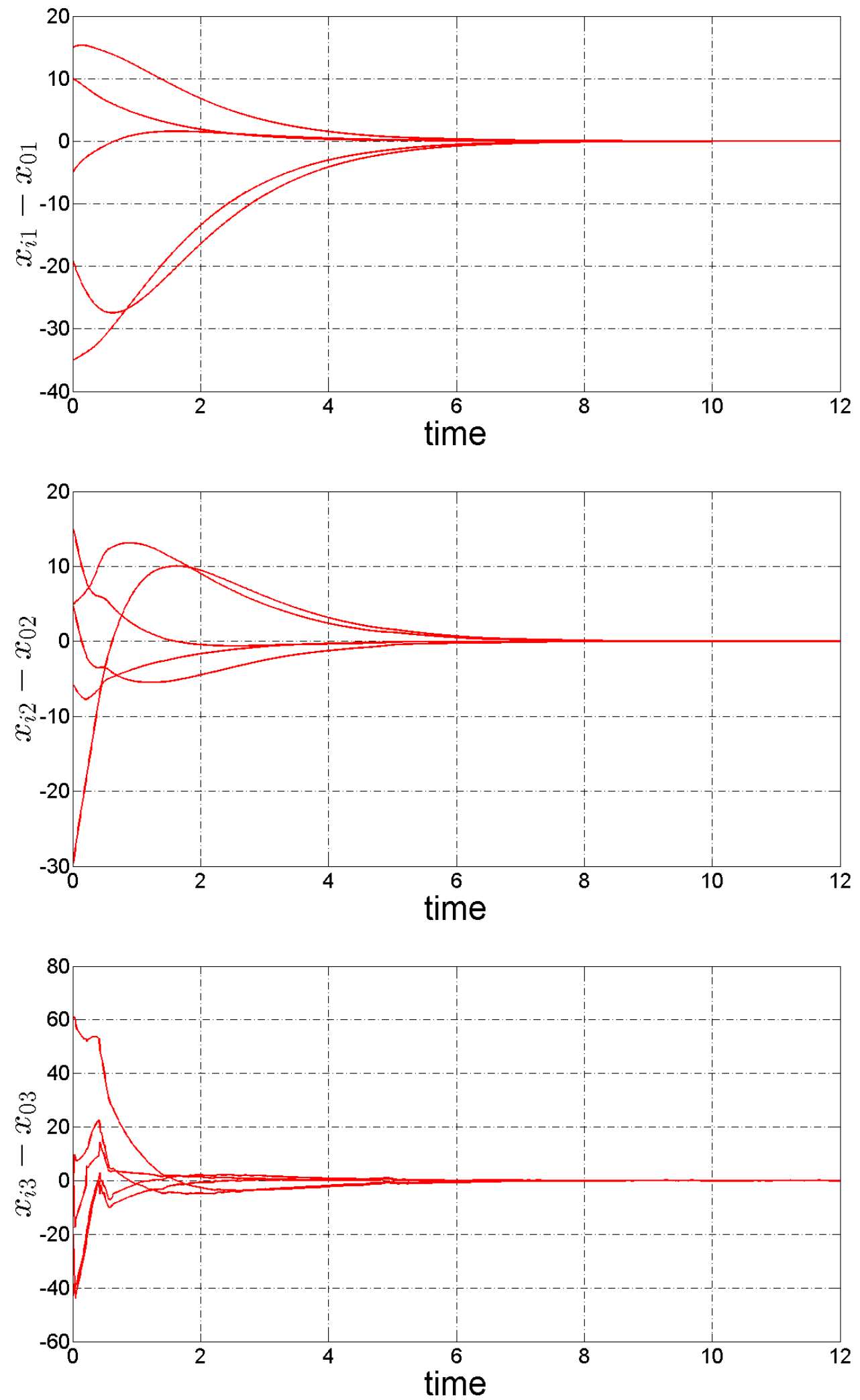

Figure 3.20: Tracking errors under control protocols (3.27), (3.30) and (3.31) with $c=60$ 


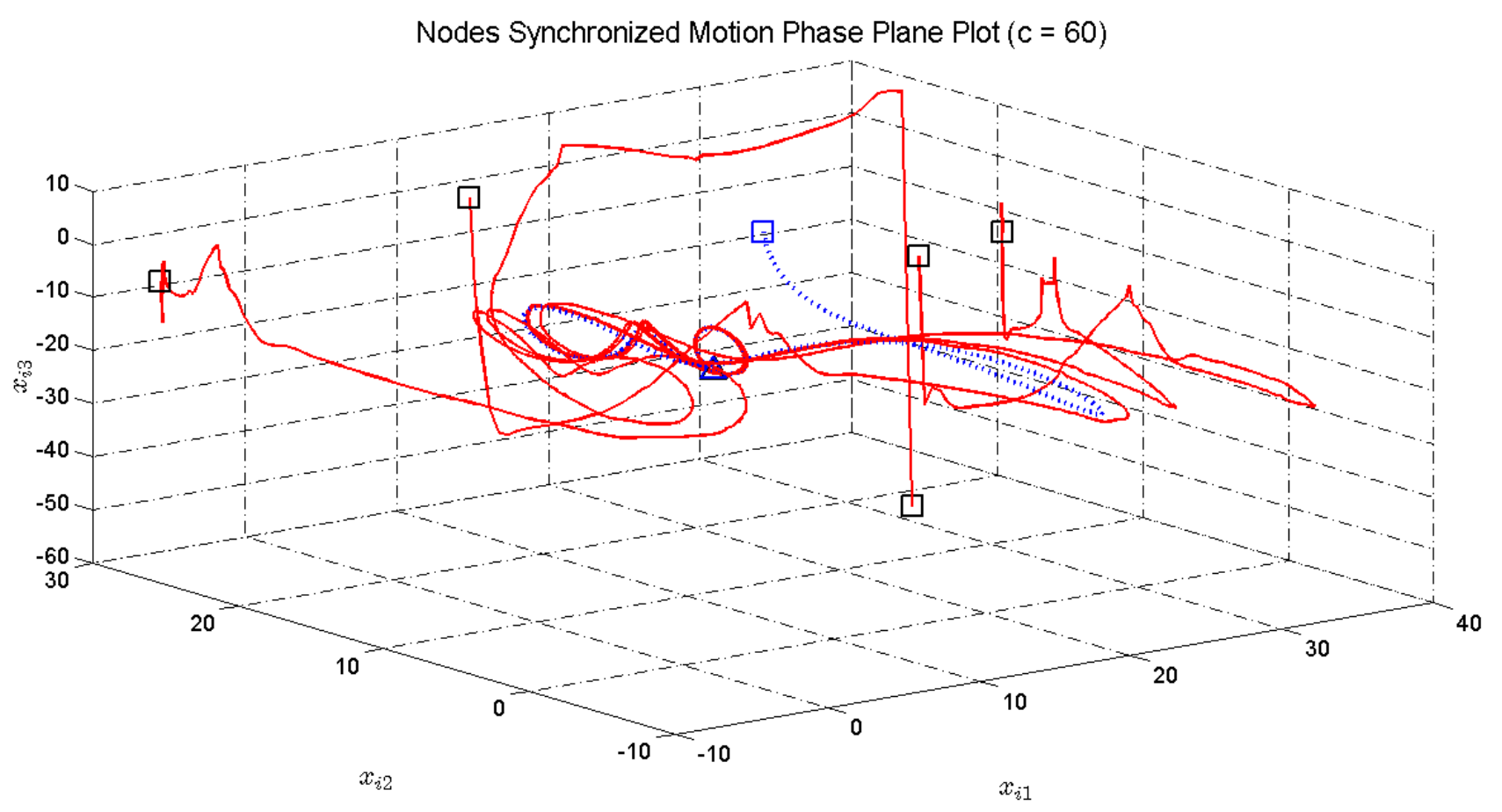

Figure 3.21: Phase trajectories of the leader agent and the follower agents under control protocols (3.27), (3.30) and (3.31) with $c=60$ 

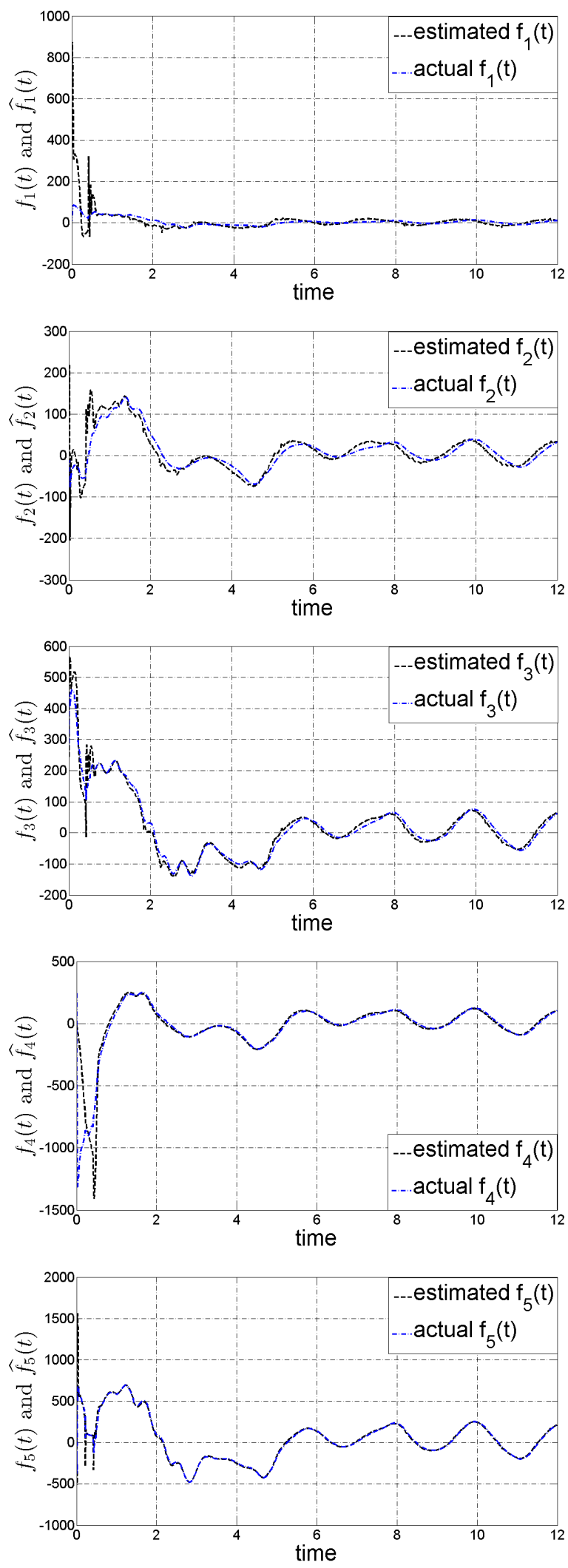

Figure 3.22: Strongly-connected directed graph: approximation of $f_{i}\left(x_{i}\right)$ (blue dashed-dotted lines) by RBFNNs (black dashed lines) for the 5 follower agents under control protocols (3.27), (3.30) and (3.31) with $c=60$ 


\section{B. Example 2}

In this work, the multiple Lyapunov function approach is used to establish the results for switching directed communication topology in Section 3.4. It is easy to see that, the fixed directed communication topology and switching undirected communication topology considered in Section 3.2 and Section 3.3 are special cases of the switching directed communication topology considered in Section 3.4.

However, compared with the results in Section 3.3 for siwtching undirected communication topology, an additional assumption that the set of all possible topologies $G$ is finite is imposed when using the multiple Lyapunov function proof technique to establish the results in Section 3.4. Therefore, a question raised naturally: Is this additional assumption a necessary condition for our proposed method to work? Or, would the proposed method still work (though it might need different proof technique) without this additional finiteness assumption of $G$ ?

Note that, if we consider only non-weighted (i.e., $0-1$ binary edge weights) graph communication topologies, the set of all possible topology is always finite, for a given number of agents. But when taking into consideration of weighted graph communication topologies, this set of all possible topology can definitely be infinite since the edge weights can be any nonnegative real number. As a result, the question posed in the paragraph above would arise.

Motivated by this question, in this simulation example, we investigate the performance of our proposed control protocols when the set of all possible topologies $G$ is infinite.

Let the switching strongly-connected directed graph communication topology with infinite many different topologies be represented by a switching strongly-connected directed graph as shown in Fig. 3.23. The weight of each edge is also shown in the figure, where $t$ denotes the time. The set $\left\{G_{1}, G_{2}, G_{3}, G_{4}, G_{5}, G_{6}\right\}$ represents the six different types of network topologies of the multi-agent system. It is easy to verify that all these six types of topologies are directed and strongly connected. The multi-agent system starts at topology $G_{1}$ and switches every $T=0.2$ to the next topology, with $G_{6}$ followed by $G_{1}$. Namely, the network topology is switching according to the following rule: $G_{1} \rightarrow G_{2} \rightarrow G_{3} \rightarrow G_{4} \rightarrow G_{5} \rightarrow G_{6} \rightarrow G_{1} \rightarrow G_{2} \cdots$. Note that the

time dependent edge weight terms such as $1 /(1+\exp (-t))$ ensures that the set of all possible communication topology graph $G$ is infinite. 


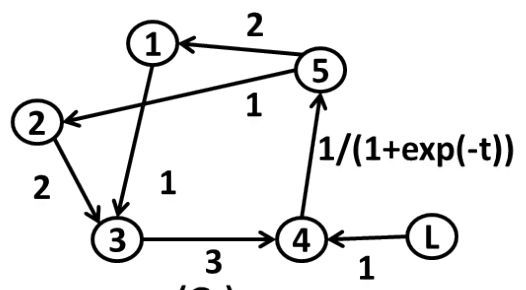

$\left(G_{1}\right)$

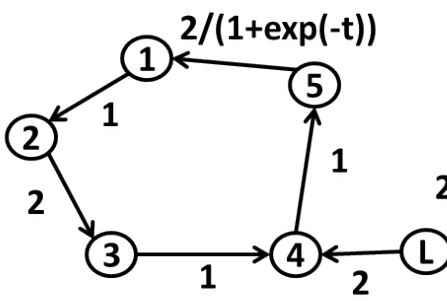

(G)

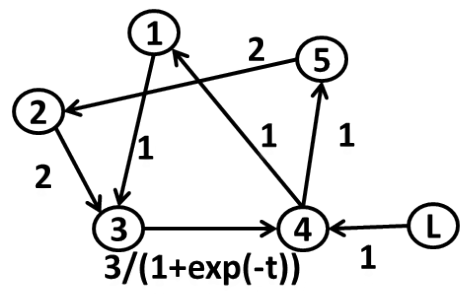

$\left(G_{5}\right)$

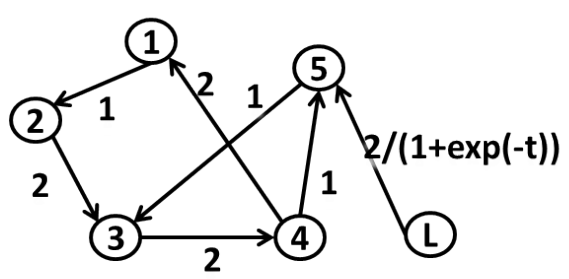

$\left(G_{2}\right)$

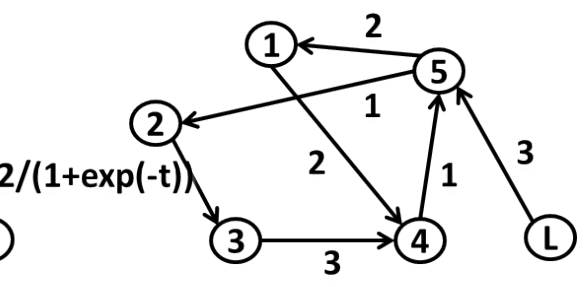

$\left(G_{4}\right)$

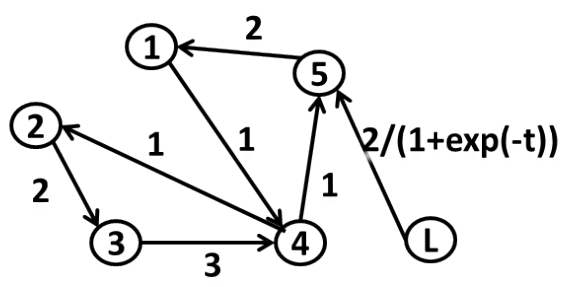

$\left(G_{6}\right)$

Figure 3.23: A switching strongly-connected directed graph communication topology with infinite many different graph

Next, with exactly the same initial condition as well as exactly the same control protocol parameter setting as Section 3.5.3, we simulate the multi-agent system using the control protocols defined by (3.27), (3.30) and (3.31) with the control gain parameter $c=60$. The state tracking error trajectories of all five follower agents are shown in Fig. 3.24, and the phase trajectories of the states of all agents are shown in Fig. 3.25 .

As we can see, as time elapses, the states of all the five follower agents synchronize to the state of the leader agent very well. The steady state synchronization errors are very close to zero.

Therefore, the simulation results presented here indicates that our proposed control protocols still works even without the assumption that the set of all possible topologies $G$ is finite. Namely, it seems that this additional assumption might probably be not necessary to guarantee the proposed control algorithm to work. On the other hand, to prove the results without this additional assumption, we might need quite different tools from the multiple Lyapunov function method employed in this chapter to prove the closed-loop 

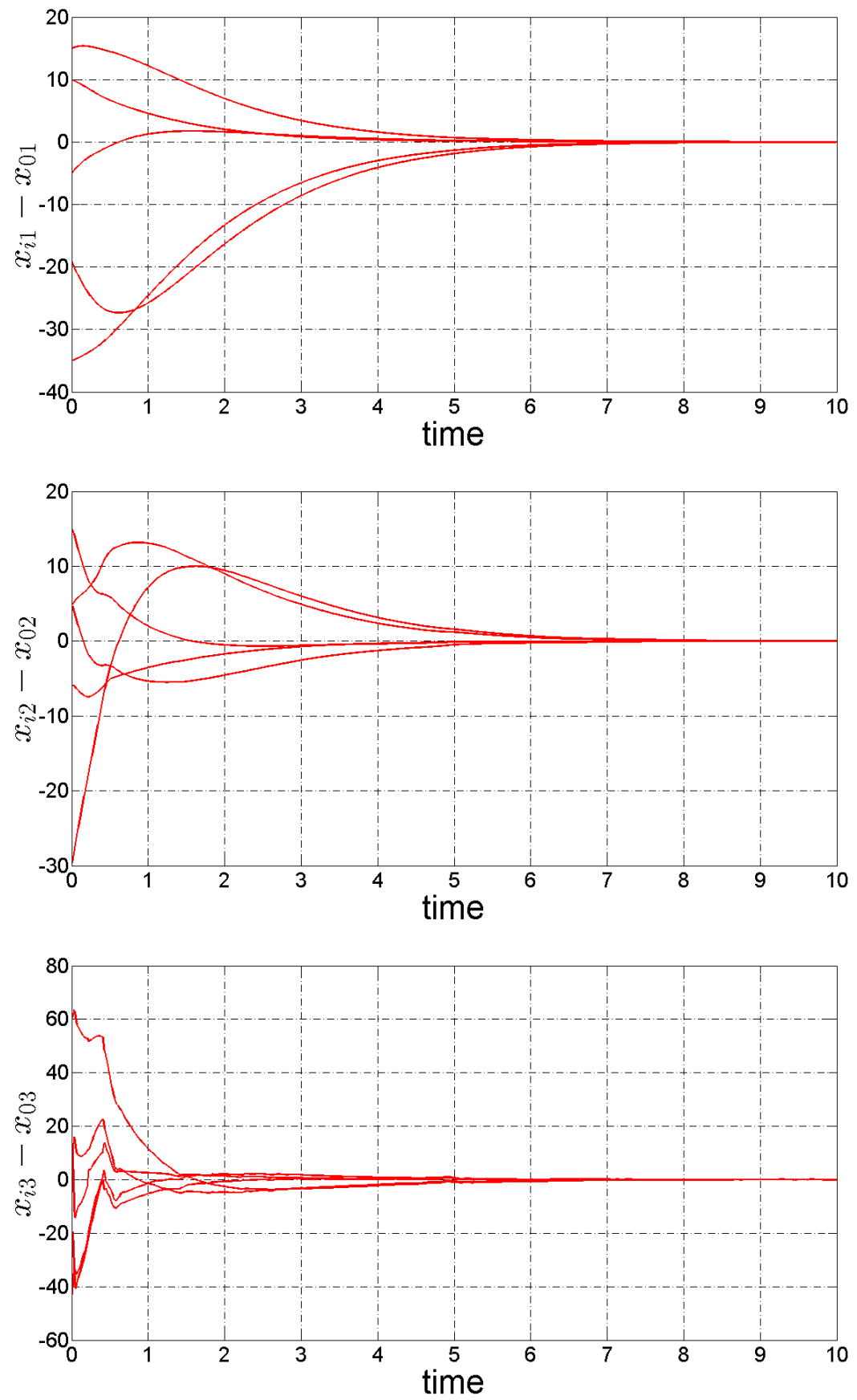

Figure 3.24: Infinite topology set $G$ case: tracking errors under control protocols (3.27), (3.30) and (3.31) with $c=60$

performance of the system under our proposed control protocols. This is because that, based on the existing literature, it is not clear how the multiple Lyapunov function method could be applied when there are infinite number of different switching Lypunov functions. This might be an interesting future research direction to explore. 


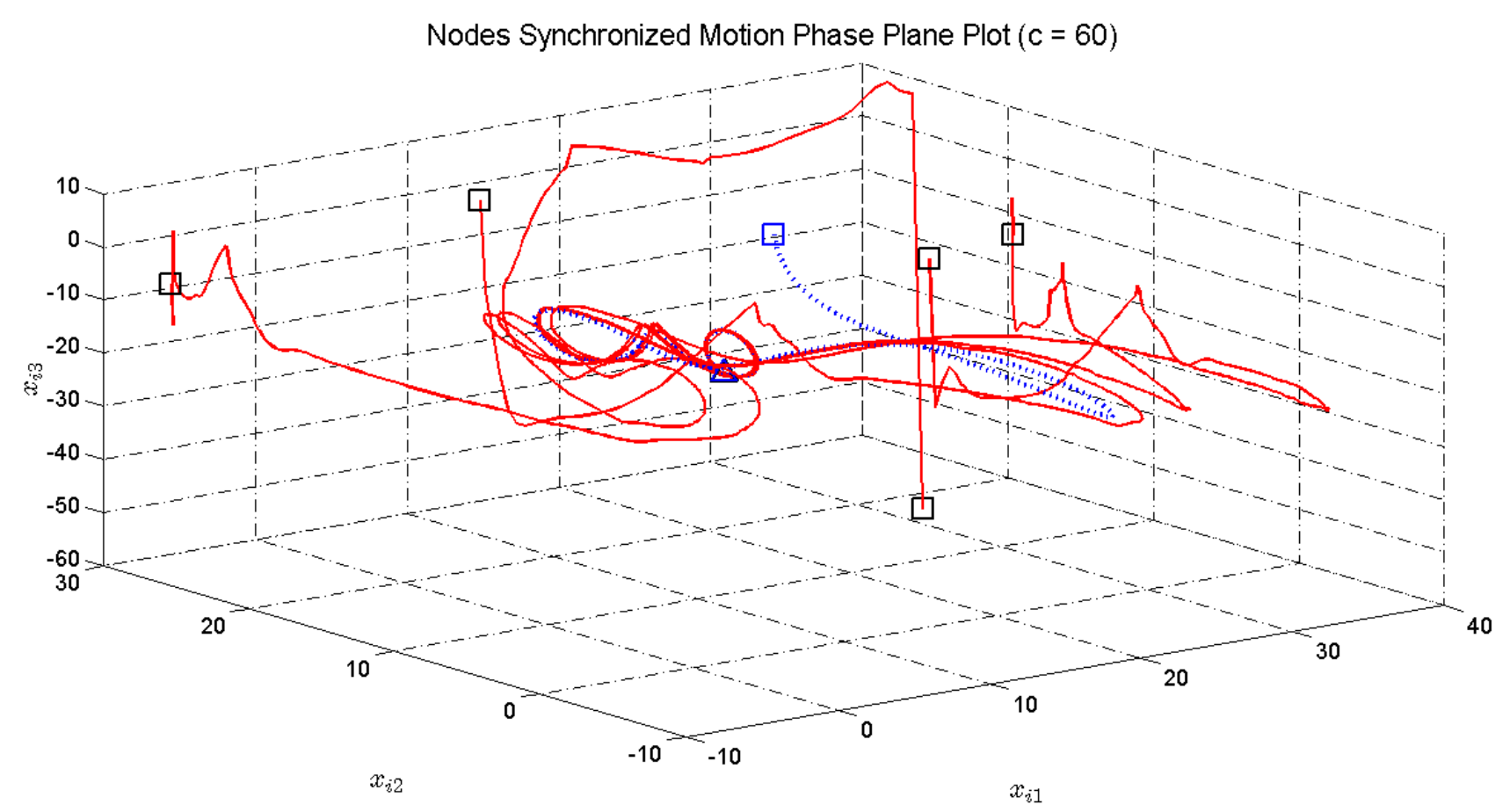

Figure 3.25: Infinite topology set $G$ case: phase trajectories of the leader agent and the follower agents under control protocols $(3.27),(3.30)$ and (3.31) with $c=60$

\subsection{Summary}

In this chapter, the distributed synchronization control of multi-agent networks with unknown nonlinearities and $n$th order agent dynamics was investigated. We first consider the case of a fixed directed interaction topology, and then extend the results to undirected switching topologies and directed switching topologies. To be specific, we proposed distributed synchronization control protocols, and through the Lyapunov functional approach, we proved that the constructed control law achieve synchronization of all follower agents to the leader agent. Numerical examples demonstrated the effectiveness of the proposed control protocols. The contributions of this study can be summarized as follows.

1. Before this work, for multi-agent network system with unknown nonlinearities, described by (3.1) and (3.2), the distributed synchronization control problem has not been studied on time varying interaction topologies. We proved that, our proposed control protocols achieve synchronization of all follower agents to the leader agent, if the time varying interaction topology is directed and strongly connected all the time, or if the time varying interaction topology is undirected and connected all the time.

2. Even for the case of a fixed interaction topology, in the previous works, only the simpler cases for agent dynamics were considered, namely, the first order and second order agent dynamics. In addition, those 
results for first order and second order agent dynamics are incorrect, due to a technical error. In our work, we have established results for more general $n$th order agent dynamics, and our results hold for both fixed and time varying interaction topologies.

3. This work demonstrates that the common Lyapunov function approach and multiple Lyapunov function approach from switch system theory are very useful to address distributed coordination control problems of multi-agent network under a time varying interaction topology. For a given multi-agent network coordination control problem, we construct a common Lyapunov function or a series of different Lyapunov function accordingly. Such approaches might be applicable to many other classes of coordination control problems for multi-agent networks with a dynamic interaction topology. 


\section{Chapter 4}

\section{Distributed Consensus Control under Jointly Connected Dynamic Interaction Topologies}

As mentioned in Section 1.5, in the second part of this thesis we will address the distributed consensus control problem for multi-agent networks with high order linear agent dynamics under jointly connected directed interaction topologies. This enriches the existing literature on multi-agent distributed consensus control under a jointly connected directed interaction topology, which was limited to very simple single integrator agent dynamics. The distributed control protocols will be developed, and the consensus will be proven.

\subsection{Problem Statement}

We consider a network of $N$ agents, with each agent described by the following higher order linear dynamic system:

$$
\dot{x_{i}}=A x_{i}+B u_{i}, i=1,2, \cdots, N
$$

where $x_{i} \in R^{n}$ is the state vector of agent $i, u_{i} \in R$ is the control input to agent $i$, and $(A, B)$ is controllable.

Without loss of generality, we can rewrite the linear dynamic system (4.1) in the following canonical form via certain coordinate transformation:

$$
\left\{\begin{aligned}
\dot{x}_{i 1} & =x_{i 2} \\
\dot{x}_{i 2} & =x_{i 3} \\
& \vdots \\
\dot{x}_{i n} & =\alpha_{1} x_{i 1}+\alpha_{2} x_{i 2}+\cdots+\alpha_{n} x_{i n}+u_{i},
\end{aligned}\right.
$$


where $x_{i}=\left[\begin{array}{llll}x_{i 1} & x_{i 2} & \cdots & x_{i n}\end{array}\right]^{\mathrm{T}}$ is the state vector of agent $i, \alpha_{1}, \alpha_{2}, \cdots, \alpha_{n}$ are scalar coefficients, and $u_{i}$ is the control input.

Let the network interaction topology be represented by a directed graph $G=(V, E(t), A(t))$. The detailed relevant notations for a directed graph $G=(V, E(t), A(t))$ is referred to Section 2.1 of this thesis.

The objective in this chapter is to design distributed control algorithm such that consensus can be achieved asymptotically by the group of agents specified by (4.2), under a jointly connected directed dynamic interaction topology. In particular, we would like to design $u_{i}$ for each agent $i$ by using only local neighborhood information of agent $i$ such that $\left(x_{i k}(t)-x_{j k}(t)\right) \rightarrow 0$ as $t \rightarrow \infty, i=1,2, \cdots, N, j=1,2, \cdots, N$, and $k=1,2, \cdots, n$.

Such a multi-agent network consensus control problem under a jointly connected directed dynamic interaction topology has once been investigated in a well known work [8] by Ren and Beard, but only the very simple first order integrator agent dynamics were considered. They proposed distributed consensus control protocols and showed that the network consensus will be achieved under a weak network topology connectivity assumption that the union of the directed interaction topology graphs contains a spanning tree frequently enough. Under this network connectivity assumption, the underlying directed dynamic interaction topology is jointly connected.

To be specific, in [8], Ren and Beard considered a network of $N$ agents with following first order integrator agent dynamics,

$$
\dot{x}_{i}=u_{i}, \quad i=1,2, \cdots, N
$$

where $x_{i}$ is the state of the agent $i$, and $u_{i}$ is the control input.

For the multi-agent system described by (4.3), Ren and Beard [8] proposed the following distributed consensus control algorithm:

$$
u_{i}=-\sum_{j=1}^{N} a_{i j}\left(x_{i}-x_{j}\right),
$$

where $a_{i j} \geq 0$ denotes the weight of the edge from agent $j$ to agent $i$ of the interaction graph.

Using graph theory and the related stochastic matrix analysis technique, Ren and Beard proved that information consensus under dynamically changing interaction topologies can be achieved asymptotically with their proposed control protocols. To be specific, their main results can be stated in the following lemma:

Lemma 6 [8] Let $t_{1}, t_{2}, \cdots$ be an infinite time sequence at which the interaction graph or weighting factors $a_{i j}$ switch and $\tau_{i}=t_{i+1}-t_{i} \in \gamma, i=0,1, \cdots$. Here, $\gamma$ is an infinite set generated from set $\hat{\tau}$, which is closed under addition, and multiplications by positive integers, with $\hat{\tau}$ being a finite set of arbitrary positive 
numbers. Let $G\left(t_{i}\right)$ be a switching interaction graph at time $t=t_{i}$ and all nonzero entries of the adjacency matrix $A=\left[a_{i j}\right]$ are lower bounded by a positive constant $\sigma_{L}$ and upper bounded by a positive constant $\sigma_{M}$. Then the distributed control protocols (4.4) achieves consensus asymptotically for the multi-agent systems specified by (4.3) if there exists an infinite sequence of uniformly bounded, nonoverlapping time intervals $\left[t_{i_{j}}, t_{i_{j}+l_{j}}\right), l_{j} \in\left[1, i_{j+1}-i_{j}\right), j=1,2, \cdots$, starting at $t_{i_{1}}=t_{0}$, with the property that each interval $\left[t_{i_{j}+l_{j}}, t_{i_{j+1}}\right)$ is uniformly bounded and the union of the directed graphs across each interval $\left[t_{i_{j}+l_{j}}, t_{i_{j+1}}\right)$ has a spanning tree. Furthermore, the consensus value is a constant. On the other hand, if the union of the graphs after some finite time does not contain a spanning tree, then consensus cannot be achieved for the multi-agent systems specified by (4.3).

Remark 3 The results stated in Lemma 6 hold for both cases of leaderless consensus and leader following consensus with a stationary leader. To be specific, if there is one agent in the group which does not have any incoming link, but the union of the interaction graphs contains a spanning tree frequently enough, then this agent must be the root of the directed spanning tree, namely, the leader. Since information consensus is guaranteed, in this case, the state of all other agents will converge to the state of the leader asymptotically.

In [15], Ren and Beard extended their work in [8] to multi-agent systems with second order integrator agent dynamics. Namely, the dynamics of each agent $i, i=1,2, \cdots, N$, are described by

$$
\left\{\begin{array}{l}
\dot{x}_{i 1}=x_{i 2} \\
\dot{x}_{i 2}=u_{i}
\end{array}\right.
$$

where $x_{i}=\left[\begin{array}{ll}x_{i 1} & x_{i 2}\end{array}\right]^{\mathrm{T}}$ is the state vector of the agent $i$, and $u_{i}$ is the control input.

For the multi-agent system described by (4.5), Ren and Beard proposed the following distributed consensus control protocols:

$$
u_{i}=-\sum_{j=1}^{N} a_{i j}\left[\left(x_{i 1}-x_{j 1}\right)+r(t)\left(x_{i 2}-x_{j 2}\right)\right]
$$

where $r(t)$ is a positive scalar at time $t$.

Their main results about the second order consensus control protocols (4.6) under switching interaction topologies are stated as follows:

Lemma 7 [15] Let $t_{1}, t_{2}, \cdots$ be the times when the directed interaction graph $G_{N}(t)$ switches. Also let $\tau$ be the dwell time such that $t_{i+1}-t_{i} \geq \tau, \forall i=0,1, \cdots$. If $G_{N}(t)$ has a directed spanning tree for each $t \in\left[t_{i}, t_{i+1}\right)$, and $\gamma$ and $\tau$ satisfies certain inequality conditions as stated in ([15]), the algorithm (4.6) achieves consensus asymptotically and is robust to perturbations under directed switching interaction topologies. 
In comparison with Lemma 6, a much stronger network connectivity assumption was made in Lemma 7 , namely, the interaction graph has a directed spanning tree at each time instant. In fact, simulation examples have been provided by Ren and Beard in [15] to illustrate that the weaker network connectivity assumption made in Lemma 6 is generally not sufficient for the proposed second order consensus algorithm (4.6) to achieve information consensus.

In this chapter, we aim at designing new distributed consensus control algorithm and extending the results in Lemma 6 to higher order systems. We will show that, under our proposed control protocols, information consensus can be achieved asymptotically with the same network connectivity assumption that the union of the switching directed interaction graphs contains a spanning tree frequently enough.

\subsection{Consensus Control Protocols and the Closed-loop System Per- formance Analysis}

Before stating our main results, we would like to mention the following fact which is important for control design and analysis in this section.

Lemma 8 [53] Let $x(t)$ be a smooth function of $t$ and assume that $x(0), \dot{x}(0), \cdots, x^{(n-1)}(0)$ are all bounded. Define

$$
s(x, t)=\left(\lambda+\frac{d}{d t}\right)^{n-1} x(t)
$$

where $\lambda>0$ is a constant. If there exists a scalar $\Phi>0$ such that $|s(x(t), t)| \leq \Phi$ for all $t \geq 0$, then there exists a finite $T_{0}$, dependent on the values of $x(0), \dot{x}(0), \cdots, x^{(n-1)}(0)$, such that

$$
\left\|x^{(i)}(t)\right\| \leq \frac{2^{i} \Phi}{\lambda^{n-i-1}}, \quad t \geq T_{0}, i=0,1, \cdots, n-1
$$

\subsubsection{Consensus Control Protocols and the Closed-loop System Performance}

For the multi-agent systems specified by (4.2), in order to achieve information consensus asymptotically, we propose the following distributed control laws for each agent $i, i=1,2, \cdots, N$,

$$
\begin{aligned}
u_{i}= & -\sum_{j=1}^{N} a_{i j}\left(z_{i}-z_{j}\right)-\left(C_{n-1}^{0} \lambda^{n-1} x_{i 2}+C_{n-1}^{1} \lambda^{n-2} x_{i 3}+\cdots+C_{n-1}^{n-2} \lambda x_{i n}\right) \\
& -\left(\alpha_{1} x_{i 1}+\alpha_{2} x_{i 2}+\cdots+\alpha_{n} x_{i n}\right)
\end{aligned}
$$


where

$$
\begin{aligned}
z_{i} & =\left(\lambda+\frac{d}{d t}\right)^{n-1} x_{i 1} \\
& =C_{n-1}^{0} \lambda^{n-1} x_{i 1}+C_{n-1}^{1} \lambda^{n-2} x_{i 2}+\cdots+C_{n-1}^{n-2} \lambda x_{i(n-1)}+C_{n-1}^{n-1} x_{i n},
\end{aligned}
$$

with $\lambda>0$ being a positive constant, and $C_{i}^{j}$,s being coefficients of the binomial expansion.

Then our main results on the information consensus of the group of agents under the proposed control protocols can be stated in the following theorem.

Theorem 4 Consider a multi-agent system of $N$ agents whose dynamics are described by (4.2) and steered by the control protocols specified by (4.7) and (4.8). Let $t_{1}, t_{2}, \cdots$ be an infinite time sequence at which the interaction graph or weighting factors $a_{i j}$ switch and $\tau_{i}=t_{i+1}-t_{i} \in \gamma, i=0,1, \cdots$. Here, $\gamma$ is an infinite set generated from set $\hat{\tau}$, which is closed under addition, and multiplications by positive integers, with $\hat{\tau}$ being a finite set of arbitrary positive numbers. Let $G\left(t_{i}\right)$ be a switching interaction graph at time $t=t_{i}$ and all nonzero entries of the adjacency matrix $A=\left[a_{i j}\right]$ are lower bounded by a positive constant $\sigma_{L}$ and upper bounded by a positive constant $\sigma_{M}$. Then the distributed control protocols (4.7) achieves consensus asymptotically for the multi-agent systems specified by (4.2) if there exists an infinite sequence of uniformly bounded, nonoverlapping time intervals $\left[t_{i_{j}}, t_{i_{j}+l_{j}}\right), l_{j} \in\left[1, i_{j+1}-i_{j}\right), j=1,2, \cdots$, starting at $t_{i_{1}}=t_{0}$, with the property that each interval $\left[t_{i_{j}+l_{j}}, t_{i_{j+1}}\right)$ is uniformly bounded and the union of the directed graphs across each interval $\left[t_{i_{j}+l_{j}}, t_{i_{j+1}}\right)$ has a spanning tree. Furthermore, the consensus is reached at a constant state $\left[\begin{array}{llll}c & 0 & \cdots & \cdots\end{array}\right]^{T}$. On the other hand, if the union of the graphs after some finite time does not contain a spanning tree, then consensus cannot be achieved for the multi-agent systems specified by (4.2).

\subsubsection{Theoretical Analysis}

Proof of Theorem 4: It is easy to verify that, for the linear dynamic system (4.2) with control input $u_{i}$ specified by (4.7) and (4.8), we have

$$
\begin{aligned}
\dot{z}_{i} & =\frac{d}{d t}\left(C_{n-1}^{0} \lambda^{n-1} x_{i 1}+C_{n-1}^{1} \lambda^{n-2} x_{i 2}+\cdots+C_{n-1}^{n-2} \lambda x_{i(n-1)}+C_{n-1}^{n-1} x_{i n}\right) \\
& =C_{n-1}^{0} \lambda^{n-1} x_{i 2}+C_{n-1}^{1} \lambda^{n-2} x_{i 3}+\cdots+C_{n-1}^{n-2} \lambda x_{i n}+C_{n-1}^{n-1} \dot{x}_{i n} \\
& =C_{n-1}^{0} \lambda^{n-1} x_{i 2}+C_{n-1}^{1} \lambda^{n-2} x_{i 3}+\cdots+C_{n-1}^{n-2} \lambda x_{i n}+\left(u_{i}+\alpha_{1} x_{i 1}+\alpha_{2} x_{i 2}+\cdots+\alpha_{n} x_{i n}\right) \\
& =-\sum_{j=1}^{N} a_{i j}\left(z_{i}-z_{j}\right),
\end{aligned}
$$


Namely, we have

$$
\dot{z}_{i}=-\sum_{j=1}^{N} a_{i j}\left(z_{i}-z_{j}\right) .
$$

It is easy to verify that the dynamic system (4.9) is exactly the same as the closed-loop system of (4.3) with control input $u_{i}$ specified by the first order consensus algorithm (4.4).

Therefore, we can apply Lemma 6 and have the conclusion that $z_{i}-z_{j}$ converges to zero asymptotically and the consensus value of $z_{i}$ is a constant, $i=1,2, \cdots, N$, and $j=1,2, \cdots, N$, if the union of the switching directed interaction graphs contains a spanning tree frequently enough. In view of (4.8), we have $z_{i}-z_{j}=\left(\lambda+\frac{d}{d t}\right)^{n-1}\left(x_{i 1}-x_{j 1}\right)$. It then follows from Lemma 8 that $\Phi$ in Lemma 8 can be chosen to be arbitrarily small since $z_{i}-z_{j} \rightarrow 0$, and we can conclude that $\left(x_{i k}-x_{j k}\right) \rightarrow 0, i=1,2, \cdots, N, j=1,2, \cdots, N$, and $k=1,2, \cdots, n$. Namely, the information consensus is achieved by the group of agents asymptotically.

By the dynamics of (4.2), the states $x_{i j}, j=1,2, \cdots, n, i=1,2, \cdots, N$, all converge to zero as $t \rightarrow \infty$. Since $z_{i}$ converges to a constant, it follows from (4.8) that $x_{i 1}, i=1,2, \cdots, N$, converges to a constant.

The proof of the second part statement of Theorem 4 (i.e., if the union of the graphs after some finite time does not contain a spanning tree, then consensus cannot be achieved) is the same as in the proof of Lemma 6 in [8].

Remark 4 Theorem 4 holds for both cases of leaderless consensus and leader following consensus with a stationary leader. Such a conclusion could be drawn based on a similar argument as used in Remark 3.

\subsubsection{Discussion}

The contribution of this work is that the results in [8], which are limited to first order integrator dynamics, are extended to $n$th order controllable linear dynamic systems, with the same network connectivity assumption being made that the union of the switching directed interaction graphs contains a spanning tree frequently enough. The results are much more general than existing literature and will be important in many applications.

Obviously, the results in [8] can be thought of as a special case of the results in Theorem 4, and all the assumptions required in Theorem 4 are the same as those made in [8]. This can be seen by that, for first order systems, $z_{i}$ is reduced to $x_{i}$, and our proposed control protocols (4.7) and (4.8) are reduced to the consensus algorithm (4.4) proposed in [8].

When compared with the second order consensus algorithm (4.6) proposed in [15], we can find that our proposed control protocols (4.7) and (4.8) require only much weaker assumptions (connectivity, dwell time, etc.) to guarantee information consensus than the algorithm (4.6). To make the algorithm comparison more 
straightforward, for second order systems (4.5), our proposed control protocols (4.7) and (4.8) are reduced to

$$
u_{i}=-\sum_{j=1}^{N} a_{i j}\left(\lambda\left(x_{i 1}-x_{j 1}\right)+\left(x_{i 2}-x_{j 2}\right)\right)-\lambda x_{i 2} .
$$

By comparing (4.10) and (4.6) and setting $\lambda=\frac{1}{\gamma}$, it is easy to know that it is the term $-\lambda x_{i 2}$ that makes the difference about the convergence performance between our proposed consensus algorithm and algorithm (4.6).

Reference [17] proposed a Lyapunov-based approach and solved the second order consensus control problem with a stationary leader under switching jointly connected interaction topologies. For a straightforward comparison, the proposed second order consensus algorithm in [17] is as follows,

$$
u_{i}=-\sum_{j=1}^{N}\left(a_{i j}\left(x_{i 1}-x_{j 1}\right)\right)-b_{i}\left(x_{i 1}-x_{01}\right)-\kappa x_{i 2},
$$

where $x_{0}=\left[\begin{array}{ll}x_{01} & x_{02}\end{array}\right]^{\mathrm{T}}$ denotes the state of the stationary leader with $x_{01}$ being a constant and $x_{02} \equiv 0, b_{i}$ is the weight of the edge of agent $i$ to the leader, and $\kappa$ is a positive constant satisfying a certain inequality as specified in [17]. The results in [17] can also be thought of as a special case of our more general results in this chapter, in the sense that they considered only undirected interaction topologies and they made some more restrictive assumptions about the interaction graph weighting factors. It is not obvious how their method can be extended to leaderless consensus control case, directed interaction topologies or higher order systems.

Compared with reference [24] which concerns with undirected interaction topologies, Theorem 4 is applicable to directed interaction topologies. Besides, Theorem 4 does not require matrix $A$ to be marginally stable. Our extension of [24] is made possible by a different structure of the control algorithm.

\subsection{Simulation Results}

\subsubsection{Consensus without a Leader}

We consider a network of five third-order systems

$$
\left\{\begin{array}{l}
\dot{x}_{i 1}=x_{i 2}, \\
\dot{x}_{i 2}=x_{i 3}, \\
\dot{x}_{i 3}=u_{i}, i=1,2, \cdots, 5,
\end{array}\right.
$$

where $u_{i}$ is the control input. 


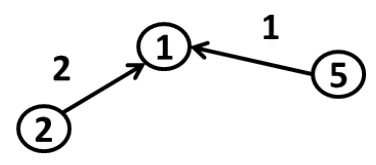

(3) $\longleftarrow 3$ (4)

$\left(G_{a}\right)$

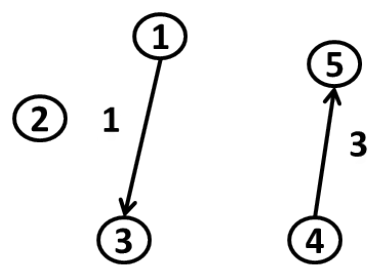

$\left(G_{c}\right)$

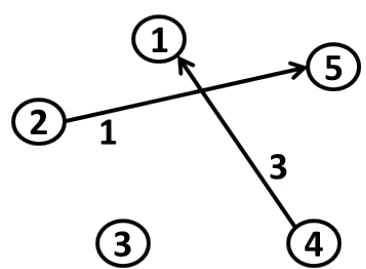

$\left(G_{e}\right)$

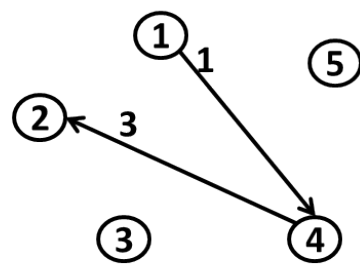

$\left(G_{b}\right)$

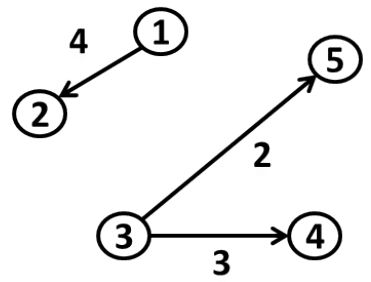

$\left(G_{d}\right)$

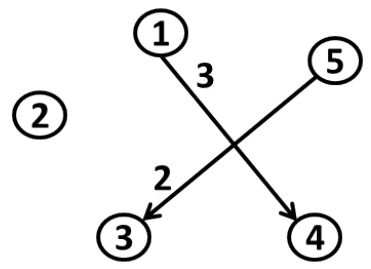

$\left(G_{f}\right)$

Figure 4.1: A switching directed graph interaction topology without a leader

Let the communication topology be represented by a switching directed graph as shown in Fig. 4.1. The weight of each edge is also shown in the figure. The set $\left\{G_{\mathrm{a}}, G_{\mathrm{b}}, G_{\mathrm{c}}, G_{\mathrm{d}}, G_{\mathrm{e}}, G_{\mathrm{f}}\right\}$ represents the six different network topologies of the multi-agent system. The multi-agent system starts at topology $G$ a and switches every $T=0.1$ to the next topology, with $G_{\text {f }}$ followed by $G_{\text {a }}$. Namely, the network topology is switching according to the following rule: $G_{\mathrm{a}} \rightarrow G_{\mathrm{b}} \rightarrow G_{\mathrm{c}} \rightarrow G_{\mathrm{d}} \rightarrow G_{\mathrm{e}} \rightarrow G_{\mathrm{f}} \rightarrow G_{\mathrm{a}} \rightarrow G_{\mathrm{b}} \cdots$. As we can see, at any given time instant, the directed interaction topology does not have a spanning tree. It is easy to verify that the union of the directed interaction graphs contains a spanning tree frequently enough for the interaction topology shown in Fig 4.1.

The initial states of the five follower agents are $x_{1}^{(0)}=\left[\begin{array}{lll}10 & 25 & 3\end{array}\right]^{\mathrm{T}}, x_{2}^{(0)}=\left[\begin{array}{lll}-20 & 15 & 10\end{array}\right]^{\mathrm{T}}, x_{3}^{(0)}=\left[\begin{array}{lll}30 & 15 & -10\end{array}\right]^{\mathrm{T}}$, $x_{4}^{(0)}=\left[\begin{array}{lll}-4 & -20-12\end{array}\right]^{\mathrm{T}}$, and $x_{5}^{(0)}=\left[\begin{array}{ll}25 & 46\end{array}\right]^{\mathrm{T}}$. The parameter $\lambda$ in our proposed consensus control algorithm (4.7) and (4.8) is set to be 1. 

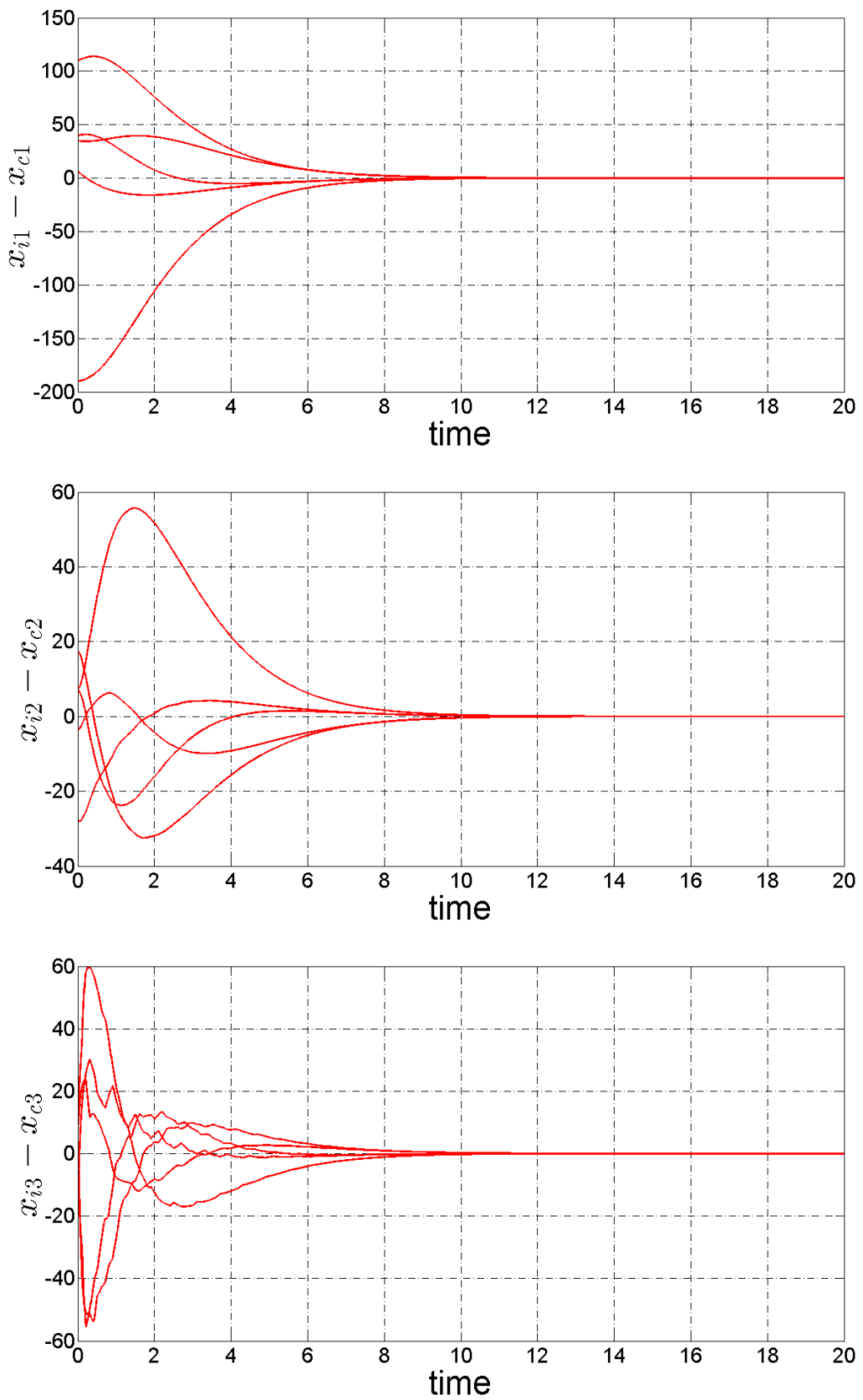

Figure 4.2: Errors between states of the agents and their centroid

Simulation results are shown in Fig. 4.2. As we can see, the states of the five agents converges to each other. 


\subsubsection{Consensus with a Stationary Leader}

We consider a network of five follower agents and one stationary leader agent. Each follower agent $i$, $i=1,2, \cdots, 5$, is a third-order system specified as follows,

$$
\left\{\begin{array}{l}
\dot{x}_{i 1}=x_{i 2} \\
\dot{x}_{i 2}=x_{i 3} \\
\dot{x}_{i 3}=u_{i}
\end{array}\right.
$$

where $u_{i}$ is the control input to agent $i$.

The dynamics of the stationary leader agent are as follows,

$$
\left\{\begin{array}{l}
\dot{x}_{01}=x_{02} \\
\dot{x}_{02}=x_{03} \\
\dot{x}_{03}=0
\end{array}\right.
$$

where $x_{0}=\left[\begin{array}{lll}x_{01} & x_{02} & x_{03}\end{array}\right]^{\mathrm{T}}$ denotes the state of the stationary leader with $x_{01}$ being a constant, $x_{02} \equiv 0$ and $x_{03} \equiv 0$.

Let the communication topology be represented by a switching directed graph as shown in Fig. 4.3. The weight of each edge is also shown in the figure. The set $\left\{G_{\mathrm{a}}, G_{\mathrm{b}}, G_{\mathrm{c}}, G_{\mathrm{d}}, G_{\mathrm{e}}, G_{\mathrm{f}}\right\}$ represents the six different network topologies of the multi-agent system. The multi-agent system starts at topology $G_{\text {a }}$ and switches every $T=0.1$ to the next topology, with $G_{\mathrm{f}}$ followed by $G_{\text {a }}$. Namely, the network topology is switching according to the following rule: $G_{\mathrm{a}} \rightarrow G_{\mathrm{b}} \rightarrow G_{\mathrm{c}} \rightarrow G_{\mathrm{d}} \rightarrow G_{\mathrm{e}} \rightarrow G_{\mathrm{f}} \rightarrow G_{\mathrm{a}} \rightarrow G_{\mathrm{b}} \cdots$. As we can see, at any given time instant, the directed interaction topology does not have a spanning tree. It is easy to verify that the union of the directed interaction graphs contains a spanning tree frequently enough for the interaction topology shown in Fig 4.3.

The initial states of the five follower agents are $x_{1}^{(0)}=\left[\begin{array}{lll}15 & 20 & 36\end{array}\right]^{\mathrm{T}}, x_{2}^{(0)}=\left[\begin{array}{lll}-20 & -8 & 15\end{array}\right]^{\mathrm{T}}, x_{3}^{(0)}=\left[\begin{array}{lll}40 & 25 & -10\end{array}\right]^{\mathrm{T}}$, $x_{4}^{(0)}=\left[\begin{array}{lll}-4 & -50 & -18\end{array}\right]^{\mathrm{T}}$, and $x_{5}^{(0)}=\left[\begin{array}{lll}25 & 14 & 6\end{array}\right]^{\mathrm{T}}$. The initial state of the stationary leader agent is $x_{0}^{(0)}=\left[\begin{array}{lll}15 & 0 & 0\end{array}\right]^{\mathrm{T}}$. The parameter $\lambda$ in our proposed consensus control algorithm (4.7) and (4.8) is set to be 1 .

The state tracking error of all the five follower agents are shown in Fig. 4.4. As we can see, under our proposed distributed control protocols, the states of all the five follower agents achieve consensus asymptotically, with the consensus value being equal to the state of the stationary leader agent. 

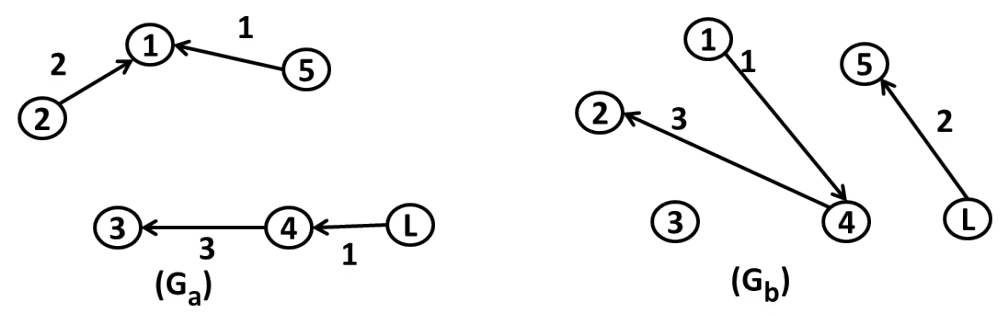

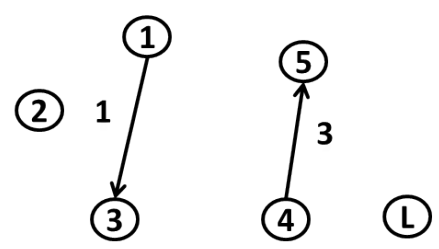

$\left(G_{c}\right)$

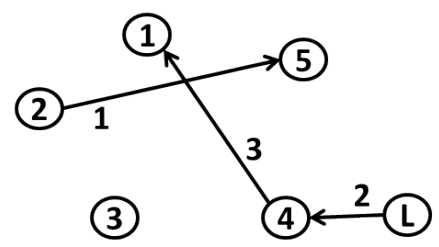

$\left(G_{e}\right)$

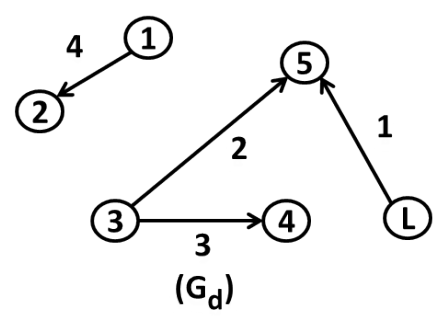

(2)

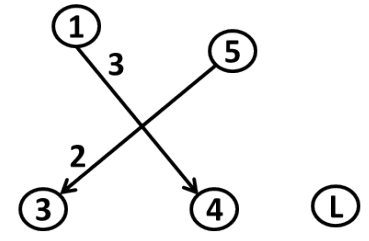

$\left(G_{f}\right)$

Figure 4.3: A switching directed graph interaction topology with a stationary leader

\subsection{Summary}

In this chapter, we considered the distributed control problem of information consensus for multi-agent systems with higher order controllable linear agent dynamics under dynamically changing directed interaction topologies and weighting factors. In particular, we proposed new consensus control protocols and extended the first order system results in [8] to $n$th order linear controllable dynamic systems. We show that, information consensus can be achieved asymptotically by the group of agents under our proposed control protocols if the union of the directed interaction graphs contains a spanning tree frequently enough. Such results are much more general than existing results in the literature. Numerical simulation illustrates the effectiveness of our proposed control algorithm. 

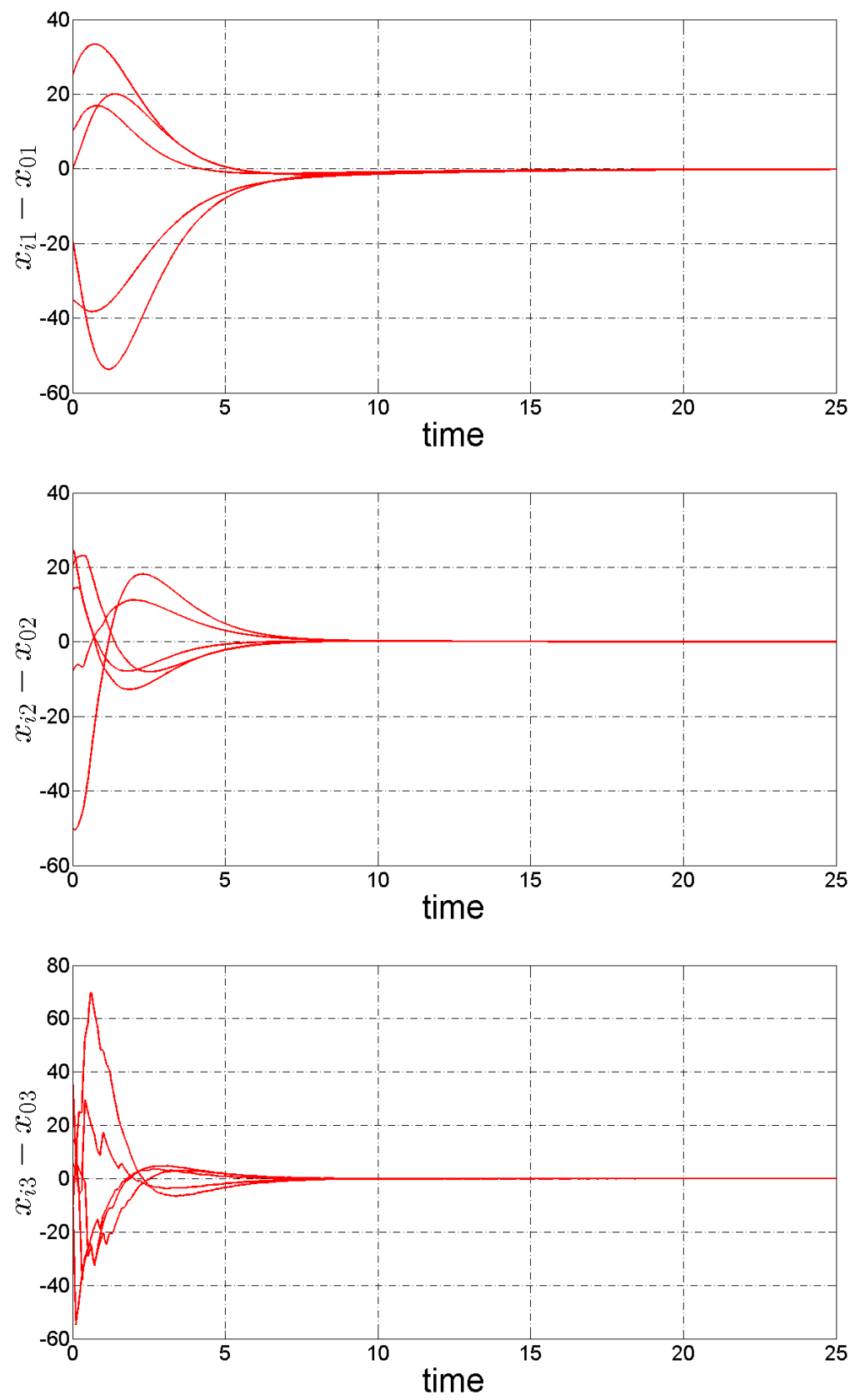

Figure 4.4: Agents' state tracking errors with a stationary leader 


\section{Chapter 5}

\section{Distributed Coordinated Tracking under State Dependent Dynamic Interaction Topologies}

As mentioned in Section 1.5, in the third part of this thesis we will address the distributed coordinated tracking problem for a multi-agent network under a state dependent jointly connected dynamic interaction topology.

\subsection{Problem Statement}

We consider a multi-agent network of $N$ follower agents and one virtual leader moving in $n$ dimensional space. The dynamics of each follower agent $i, i=1,2, \cdots, N$, are described by

$$
\left\{\begin{array}{c}
\dot{q}_{i}=p_{i} \\
\dot{p}_{i}=u_{i}
\end{array}\right.
$$

where $x_{i}=\left[\begin{array}{ll}q_{i}^{\mathrm{T}} & p_{i}^{\mathrm{T}}\end{array}\right]^{\mathrm{T}} \in R^{2 n}$ denotes the state of agent $i$, and $u_{i}$ is its control input.

The dynamics of the virtual leader are described by

$$
\left\{\begin{array}{l}
\dot{q}_{\gamma}=p_{\gamma} \\
\dot{p}_{\gamma}=0
\end{array}\right.
$$

where $x_{\gamma}=\left[q_{\gamma}^{\mathrm{T}} p_{\gamma}^{\mathrm{T}}\right]^{\mathrm{T}} \in R^{2 n}$ describes the state of the virtual leader. 
For notational convenience, we also define

$$
q=\left[\begin{array}{c}
q_{1} \\
q_{2} \\
\vdots \\
q_{N}
\end{array}\right], p=\left[\begin{array}{c}
p_{1} \\
p_{2} \\
\vdots \\
p_{N}
\end{array}\right]
$$

Assume that each agent has only a limited communication capability and can only communicate with agents within its neighborhood. The neighbor set of agent $i$ at time $t$ is denoted as,

$$
N_{i}(t)=\left\{j:\left\|q_{i}-q_{j}\right\| \leq r, j=1,2, \cdots, N, j \neq i\right\}
$$

where $\|\cdot\|$ is the Euclidean norm. Thus, we have assumed that all agents have an identical influencing/sensing radius of $r$.

Note that the neighbor set of each agent may change over time because the distance between agents may vary over time. We define the undirected neighborhood graph as $G_{N}(t)=(V, E(t))$, where $V=\left\{v_{1}, v_{2}, \cdots, v_{N}\right\}$ is a set of $N$ vertices, each denoting a follower agent, and $E(t) \subset V \times V$ is the set of edges of the graph at time $t$. An edge $\left(v_{i}, v_{j}\right) \in E(t)$ denotes that $\left\|q_{i}(t)-q_{j}(t)\right\| \leq r$, namely, agent $j$ and agent $i$ are within the neighborhood of each other at time $t$. In this situation, agent $i$ and agent $j$ are also said to be adjacent at time $t$. Define the adjacency matrix as $A=\left[a_{i j}(t)\right]$ with $a_{i j}(t) \geq 0$ if $\left(v_{j}, v_{i}\right) \in E(t)$ and $a_{i j}(t)=0$ otherwise. Define the graph Laplacian $L(t)$ as $L(t)=D(t)-A(t)$, where $D(t)$ is the diagonal in-degree matrix with the $i$ th diagonal element being $\sum_{j=1}^{N} a_{i j}(t)$. A path of length $l$ is a sequence of edges $\left(v_{i_{1}}, v_{i_{2}}\right),\left(v_{i_{2}}, v_{i_{3}}\right), \cdots,\left(v_{i_{l}}, v_{i_{l+1}}\right)$ with all these $l+1$ vertices being distinct from each other.

In 2006, Olfati-Saber [9] proposed distributed control protocols of the following form,

$$
\begin{aligned}
u_{i}= & \sum_{\substack{j \in N_{i}(t)\\
}} \nabla_{q_{i}} \psi_{\alpha}\left(\left\|q_{j}-q_{i}\right\|_{\sigma}\right)+\sum_{j \in N_{i}(t)} a_{i j}(t)\left(p_{j}-p_{i}\right)-\left(c_{1}\left(q_{i}-q_{\gamma}\right)+c_{2}\left(p_{i}-p_{\gamma}\right)\right) \\
& c_{1}>0, c_{2}>0
\end{aligned}
$$

where $\|z\|_{\sigma}$ is the $\|\cdot\|_{\sigma}$ norm of the vector $z$ defined as $\|z\|_{\sigma}=\frac{1}{\epsilon}\left[\sqrt{1+\epsilon\|z\|^{2}}-1\right]$ with a parameter $\epsilon>0$. The gradient $\nabla_{q_{i}} \psi_{\alpha}\left(\left\|q_{j}-q_{i}\right\|_{\sigma}\right)$ is a nonlinear term derived from an artificial potential function. To be specific, $\psi_{\alpha}(z)$ is a smooth pairwise attractive/repulsive potential with a finite cut-off at $r_{\alpha}=\|r\|_{\sigma}$ and a 
global minimum at $z=d_{\alpha}=\|d\|_{\sigma}$. Mathematically, $\psi_{\alpha}(z)$ is defined as follows,

$$
\psi_{\alpha}(z)=\int_{d_{\alpha}}^{z} \phi_{\alpha}(s) d s
$$

with

$$
\phi_{\alpha}(z)=\frac{1}{2} \rho_{h}\left(z / r_{\alpha}\right)\left[(a+b) \frac{z-d_{\alpha}+c}{\sqrt{1+\left(z-d_{\alpha}+c\right)^{2}}}+a-b\right]
$$

where the parameters satisfy $0<a \leq b, c=\frac{|a-b|}{\sqrt{4 a b}}$, and the bump function $\rho_{h}(z), h \in(0,1)$, is defined as

$$
\rho_{h}(z)= \begin{cases}1, & \text { if } z \in[0, h) \\ 0.5\left[1+\cos \left(\pi \frac{z-h}{1-h}\right)\right], & \text { if } z \in[h, 1] \\ 0, & \text { otherwise. }\end{cases}
$$

The adjacency weight function $a_{i j}(t)$ is also a nonlinear function of the states of the agents defined as

$$
a_{i j}(t)= \begin{cases}0, & \text { if } j=i \\ \rho_{h}\left(\left\|q_{j}-q_{i}\right\|_{\sigma} /\|r\|_{\sigma}\right), & \text { if } j \neq i\end{cases}
$$

Under the control protocols (5.4), Olfati-Saber showed that all the follower agents would be driven to track the virtual leader. The control protocols (5.4) indicate that all the follower agents are informed, that is, all the agents have access to the information of the virtual leader.

In 2009, $\mathrm{Su}$ et al. [22] extended the results in [9] to the case that only a fraction of the follower agents are informed. To be specific, they proposed the following control protocols,

$$
\begin{aligned}
u_{i}= & \sum_{\substack{j \in N_{i}(t) \\
c_{1}>0, c_{2}>0}} \nabla_{q_{i}} \psi_{\alpha}\left(\left\|q_{j}-q_{i}\right\|_{\sigma}\right)+\sum_{j \in N_{i}(t)} a_{i j}(t)\left(p_{j}-p_{i}\right)-h_{i}\left(c_{1}\left(q_{i}-q_{\gamma}\right)+c_{2}\left(p_{i}-p_{\gamma}\right)\right), \\
& c_{1},
\end{aligned}
$$

where $h_{i}=1$ if agent $i$ is informed and $h_{i}=0$ otherwise.

Then, using the LaSalle Invariance Principle and the artificial potention functional approach, Su et al. showed that such control protocols enable all the informed agents to move with the desired velocity, and an uninformed agent to also move with the same desired velocity if it can be influenced by the informed agents from time to time during the evolution. It is also shown that the distance between any informed follower agent and the virtual leader is bounded for all $t \geq 0$. 
Note that both the control protocols (5.4) and the control protocols (5.5) are nonlinear due to the

appearance of the gradient term $\sum_{j \in N_{i}(t)} \nabla_{q_{i}} \psi_{\alpha}\left(\left\|q_{j}-q_{i}\right\|_{\sigma}\right)$ and an adjacency weight function term $a_{i j}(t)$, both of which are rather complicated nonlinear functions of the agent states. Moreover, in both [9] and [22], the informed agents are assumed to have access to both the position and velocity information of the virtual leader, and topology connectivity preserving was not considered.

In this chapter, we will design a much simpler distributed controller to achieve virtual leader tracking (flocking, consensus tracking) objectives, both in the presence and in the absence of the virtual leader velocity information. In addition, we will propose a new connectivity enhancing mechanism that is effective in maintaining not only the initially existent topology edges but also those newly formed edges that were not initially existent.

\subsection{Virtual Leader Tracking Control Protocols and the Closed- loop System Performance Analysis}

In this section, we first propose new distributed control protocols that are much simpler than those in [9] and [22], and show that the virtual leader tracking objective will be achieved (Section 5.2.1). Then we analyze the performance of our proposed control protocols in the absence of the virtual leader velocity information (Section 5.2.2). After that, we propose a new connectivity enhancing mechanism which is effective in improving the topology connectivity to help achieve the tracking objective (Section 5.2.3).

\subsubsection{Control Protocols and the Closed-loop System Performance Analysis with Virtual Leader Velocity Information}

In this section, we make the same assumption as in [22] that only some, but not all, of the follower agents are informed agents who are given the position and velocity information of the virtual leader. Then, we propose distributed control protocols that are much simpler than those in [9] and [22] and show that they achieve the virtual leader tracking control objective.

More specifically, we will propose the following distributed control protocol for each agent $i, i=1,2, \cdots, N$,

$$
\begin{aligned}
u_{i}= & k_{1} \sum_{j \in N_{i}(t)} a_{i j}(t)\left(q_{j}-q_{i}\right)+k_{2} \sum_{j \in N_{i}(t)} a_{i j}(t)\left(p_{j}-p_{i}\right)-h_{i}\left(c_{1}\left(q_{i}-q_{\gamma}\right)+c_{2}\left(p_{i}-p_{\gamma}\right)\right), \\
& k_{1}>0, k_{2}>0, c_{1}>0, c_{2}>0
\end{aligned}
$$


where $h_{i}=1$ if agent $i$ is informed and $h_{i}=0$ otherwise. Here, $k_{1}, k_{2}, c_{1}$ and $c_{2}$ are arbitrary positive constants. $a_{i j}(t)$ is the $i j$ th entry of the adjacency weight matrix network topology, with

$$
a_{i j}(t)= \begin{cases}0, & \text { if } j=i \text { or }\left\|q_{j}(t)-q_{i}(t)\right\|>r, \\ \frac{r-\left\|q_{j}-q_{i}\right\|}{\delta}, & \text { if } j \neq i \text { and }\left\|q_{j}(t)-q_{i}(t)\right\| \in(r-\delta, r], \\ 1, & \text { if } j \neq i \text { and }\left\|q_{j}(t)-q_{i}(t)\right\| \leq r-\delta,\end{cases}
$$

where $\delta \in(0, r)$ is a positive constant that can be tuned according to specific applications.

It is easy to see that our proposed control protocols (5.6) are much simpler than the control protocols (5.4) proposed in [9] and the control protocols (5.5) proposed in [22].

Without loss of generality, we assume that the first $M_{0}\left(1 \leq M_{0} \leq N\right)$ agents are informed agents, namely, $h_{i}=1$ for $i=1,2, \cdots, M_{0}$, and $h_{i}=0$ for $i=M_{0}+1, M_{0}+2, \cdots, N$. Recall that we have assumed the virtual leader to move along a fixed direction with a constant velocity $p_{d}$.

Before stating our main results, we will use the same definitions as in [22] for Type I uninformed agents and Type II uninformed agents, as follows.

Definition 8 [22] Denote the union of all neighborhood graphs across a nonempty finite time interval $\left[t_{i}, t_{i+1}\right), t_{i+1}>t_{i}$ as $\hat{G}\left(t_{i}, t_{i+1}\right)$, whose edges are the union of the edges of those neighborhood graphs. For an uninformed agent, if there is a path between this agent and one informed agent in the union $\hat{G}\left(t_{i}, t_{i+1}\right)$, then we say that there exists a joint path between the uninformed agent and the informed agent during the finite time interval $\left[t_{i}, t_{i+1}\right)$. We divide all the uninformed agents into two types. An uninformed agent is called a Type I uninformed agent if there exists an infinite sequence of contiguous, nonempty and uniformly bounded time-intervals $\left[t_{i}, t_{i+1}\right), i=0,1,2, \cdots$, such that across each time interval there exists a joint path between this agent and one informed agent. Otherwise, it is called a Type II uninformed agent.

It is important to note that a Type I uninformed agent is only required to be linked with an informed agent through a joint path during each time interval $\left[t_{i}, t_{i+1}\right)$, and not necessary to stay in touch with an informed agent through a path all the time. It is also obvious that, if the interaction topology among all the follower agents is jointly connected, then all the uninformed follower agents would be Type I uninformed agents.

To state the main results of this section, we define a positive semi-definite energy function $Q(q, p)$ as follows,

$$
Q(q, p)=\frac{1}{2} \sum_{i=1}^{N}\left(U_{i}(q)+\left(p_{i}-p_{\gamma}\right)^{\mathrm{T}}\left(p_{i}-p_{\gamma}\right)\right),
$$


where

$$
U_{i}(q)=\frac{k_{1}}{2} \sum_{j=1, j \neq i}^{N} V_{i j}\left(q_{i}, q_{j}\right)+h_{i} c_{1}\left(q_{i}-q_{\gamma}\right)^{\mathrm{T}}\left(q_{i}-q_{\gamma}\right) .
$$

with

$$
V_{i j}\left(q_{i}, q_{j}\right)= \begin{cases}\left\|q_{i}-q_{j}\right\|^{2}, & \text { if }\left\|q_{j}(t)-q_{i}(t)\right\| \leq r-\delta, \\ (r-\delta)^{2}+\frac{\frac{1}{2} r\left\|q_{i}-q_{j}\right\|^{2}-\frac{1}{3}\left\|q_{i}-q_{j}\right\|^{3}}{\delta}-\frac{\frac{1}{2} r(r-\delta)^{2}-\frac{1}{3}(r-\delta)^{3}}{\delta}, & \text { if }\left\|q_{j}(t)-q_{i}(t)\right\| \in(r-\delta, r], \\ (r-\delta)^{2}+\frac{r^{3}}{6 \delta}-\frac{\frac{1}{2} r(r-\delta)^{2}-\frac{1}{3}(r-\delta)^{3}}{\delta}, & \text { if }\left\|q_{j}(t)-q_{i}(t)\right\|>r .\end{cases}
$$

Then our main results on virtual leader tracking of the group of agents can be stated in the following theorem.

Theorem 5 Consider a multi-agent system of $N$ agents whose dynamics are described by (5.1) and steered by the control protocols specified by (5.6). The dynamics of the virtual leader are described by (5.2). Suppose that a portion of the follower agents are informed agents which can get access to the position and velocity information of the virtual leader. Suppose that the initial energy $Q_{0}:=Q(q(0), p(0))$ is finite. Then the following statements hold:

i) The distance between each informed agent and the virtual leader is not greater than $\sqrt{2 Q_{0} / c_{1}}$ for all $t \geq 0$.

ii) If the interaction topology among all the follower agents is jointly connected, then all the informed and uninformed agents will achieve asymptotic tracking of the virtual leader.

iii) In the case that the interaction topology is not necessarily jointly connected, all the informed agents and Type I uninformed agents will still achieve asymptotic tracking of the virtual leader.

Remark 5 The previous works ( [20,21]) also extended [9] to the case that only a fraction of the follower agents are informed, and their proposed control protocols work for the case that the virtual leader has a time varying velocity. However, in both [20] and [21], in order to drive the follower agents to track the virtual leader, it is required that the undirected interaction topology is connected all the time, while in Theorem 5, only joint connectivity condition is required. In addition, Part iii) of Theorem 5 also established some guaranteed desired performance of the proposed control protocols for situations where even the mild joint connectivity condition is not satisfied.

Proof of Theorem 5: We will now first prove Part i) of Theorem 5 as follows. Denote the position difference vector and the velocity difference vector between agent $i$ and the virtual leader as $\tilde{q_{i}}=q_{i}-q_{\gamma}$ and $\tilde{p}_{i}=p_{i}-p_{\gamma}$, 
respectively. Then,

$$
\left\{\begin{array}{l}
\dot{\tilde{q}}_{i}=\tilde{p}_{i}, \\
\dot{\tilde{p}}_{i}=u_{i}, i=1,2, \cdots, N .
\end{array}\right.
$$

Let $q_{i j}=q_{i}-q_{j}, \tilde{q}_{i j}=\tilde{q}_{i}-\tilde{q}_{j}, p_{i j}=p_{i}-p_{j}$ and $\tilde{p}_{i j}=\tilde{p}_{i}-\tilde{p}_{j}$. Clearly, we have $\tilde{q}_{i j}=q_{i j}$ and $\tilde{p}_{i j}=p_{i j}$.

Therefore, the control protocols (5.6) can be rewritten as

$$
u_{i}=k_{1} \sum_{j \in N_{i}(t)} a_{i j}\left(\tilde{q}_{j}-\tilde{q}_{i}\right)+k_{2} \sum_{j \in N_{i}(t)} a_{i j}\left(\tilde{p}_{j}-\tilde{p}_{i}\right)-h_{i} c_{1} \tilde{q}_{i}-h_{i} c_{2} \tilde{p}_{i}, i=1,2, \cdots, N,
$$

and the positive semi-definite energy function (5.7) can be rewritten as

$$
Q(\tilde{q}, \tilde{p})=\frac{1}{2} \sum_{i=1}^{N}\left(U_{i}(\tilde{q})+\tilde{p}_{i}^{\mathrm{T}} \tilde{p}_{i}\right)
$$

where

$$
U_{i}(\tilde{q})=\frac{k_{1}}{2} \sum_{j=1, j \neq i}^{N} V_{i j}\left(\tilde{q_{i}}, \tilde{q_{j}}\right)+h_{i} c_{1} \tilde{q}_{i}^{\mathrm{T}} \tilde{q}_{i}
$$

and

$$
\tilde{q}=\left[\begin{array}{c}
\tilde{q}_{1} \\
\tilde{q}_{2} \\
\vdots \\
\tilde{q}_{N}
\end{array}\right], \tilde{p}=\left[\begin{array}{c}
\tilde{p}_{1} \\
\tilde{p}_{2} \\
\vdots \\
\tilde{p}_{N}
\end{array}\right]
$$

It is clear that $Q(\tilde{q}, \tilde{p})$ is a positive semi-definite function of $(\tilde{q}, \tilde{p})$. Now consider the term $V_{i}=$ $\frac{1}{2} \sum_{j=1, j \neq i}^{N} V_{i j}$. It is easy to show that

$$
\dot{V}_{i}=\sum_{j \in N_{i}(t)} a_{i j}(t)\left(\tilde{q}_{j}-\tilde{q}_{i}\right)^{\mathrm{T}}\left(\tilde{p}_{j}-\tilde{p}_{i}\right)
$$

from which, by a change of summation order, we have

$$
\begin{aligned}
\sum_{i=1}^{N} \dot{V}_{i} & =\sum_{i=1}^{N} \sum_{j \in N_{i}(t)} a_{i j}(t)\left(\tilde{q}_{j}-\tilde{q}_{i}\right)^{\mathrm{T}}\left(\tilde{p}_{j}-\tilde{p}_{i}\right) \\
& =-2 \sum_{i=1}^{N} \sum_{j \in N_{i}(t)} a_{i j}(t)\left(\tilde{q}_{j}-\tilde{q}_{i}\right)^{\mathrm{T}} \tilde{p}_{i} .
\end{aligned}
$$


Therefore, the derivative of $Q$ along the trajectories of the follower agents and the virtual leader is given by,

$$
\begin{aligned}
\dot{Q} & =\frac{1}{2} \sum_{i=1}^{N}\left(\dot{U}_{i}(\tilde{q})+2 \tilde{p}_{i}^{\mathrm{T}} \dot{\tilde{p}}_{i}\right), \\
& =-\tilde{p}^{\mathrm{T}}\left(\left(k_{2} L(t)+c_{2} H(t)\right) \otimes I_{n}\right) \tilde{p},
\end{aligned}
$$

where $L(t)$ is the Lapalacian matrix for the network topology, and $H(t)$ is a diagonal matrix whose $i$ th diagonal entry is $h_{i}$.

Since both $L(t)$ and $H(t)$ are positive semi-definite, we have $\dot{Q} \leq 0$. This implies that $Q(t)$ is a nonincreasing function for all $t \geq 0$ and thus $Q(t) \leq Q(0)$ for all $t \geq 0$. It then follows from (5.11) and (5.12) that $c_{1} \tilde{q}_{i}^{\mathrm{T}} \tilde{q}_{i} \leq 2 Q_{0}$ for any informed agent $i$. Hence the distance between an informed agent $i$ and the virtual leader is not greater than $\sqrt{2 Q_{0} / c_{1}}$ for all $t \geq 0$.

This completes the proof of Part i) of Theorem 5.

Next, we prove Parts ii) and iii) of Theorem 5.

According to Part i) of the Theorem 5, the distance between an informed agent and the virtual leader is not greater than $\sqrt{2 Q_{0} / c_{1}}$ for all $t \geq 0$, and $Q(t) \leq Q(0)$ for all $t \geq 0$. It follows from (5.11) and (5.12) that $\left\|\tilde{p_{i}}\right\| \leq \sqrt{2 Q_{0}}, i=1,2, \cdots, N$. Namely, the velocity difference between any agent and the virtual leader is not greater than $\sqrt{2 Q_{0}}$ for all $t \geq 0$. Then, by definition of Type I uninformed agent, there exists a joint path between a Type I uninformed agent and one informed agent in each finite time interval $\left[t_{i}, t_{i+1}\right)$, $i=0,1,2, \cdots$, with the definition of these time intervals $\left[t_{i}, t_{i+1}\right)$ provided in Definition 8 . Then we can easily conclude that the distance between any Type I uninformed agent and the virtual leader is also finite for all $t \geq 0$.

Let $M, M_{0} \leq M \leq N$, denote the total number of informed agents and Type I uninformed agents in the group. Without loss of generality, we label the Type I uninformed agents from $M_{0}+1$ to $M$. By definition, there exists a $T>0$, such that all the informed agents and Type I uninformed agents cannot be influenced by a Type II uninformed agents directly or indirectly for all $t \geq T$. For any $t \geq T$, we consider the following positive semi-definite energy function

$$
\hat{Q}(\hat{\tilde{q}}, \hat{\tilde{p}})=\frac{1}{2} \sum_{i=1}^{M}\left(\hat{U}_{i}(\hat{\tilde{q}})+\tilde{p}_{i}^{\mathrm{T}} \tilde{p}_{i}\right)
$$

where

$$
\hat{U}_{i}(\hat{\tilde{q}})=\frac{k_{1}}{2} \sum_{j=1, j \neq i}^{M} V_{i j}\left(\tilde{q}_{i}, \tilde{q}_{j}\right)+h_{i} c_{1} \tilde{q}_{i}^{\mathrm{T}} \tilde{q}_{i}
$$


and

$$
\hat{\tilde{q}}=\left[\begin{array}{c}
\tilde{q}_{1} \\
\tilde{q}_{2} \\
\vdots \\
\tilde{q}_{M}
\end{array}\right], \hat{\tilde{p}}=\left[\begin{array}{c}
\tilde{p}_{1} \\
\tilde{p}_{2} \\
\vdots \\
\tilde{p}_{M}
\end{array}\right] .
$$

Obviously, it follows from (5.11) and (5.15) that $\hat{Q} \leq Q$. Then following a procedure similar to the one in calculating $\dot{Q}$ in the proof of Part i), we have that the derivative of $\hat{Q}$ along the trajectories of the follower agents and the virtual leader is given by

$$
\begin{aligned}
\dot{\hat{Q}} & =\frac{1}{2} \sum_{i=1}^{M}\left(\dot{\hat{U}}_{i}(\hat{\tilde{q}})+2 \tilde{p}_{i}^{\mathrm{T}} \dot{\tilde{p}}_{i}\right), \\
& =-\hat{\tilde{\hat{p}}}^{\mathrm{T}}\left(\left(k_{2} \hat{L}(t)+c_{2} \hat{H}(t)\right) \otimes I_{n}\right) \hat{\tilde{p}}
\end{aligned}
$$

where $\hat{L}(t)=\left[\hat{l}_{i j}(t)\right]_{M \times M}$ is the corresponding Lapalacian matrix of the subgraph consisting of only informed agents and Type I uninformed agents, namely,

$$
\hat{l}_{i j}(t)= \begin{cases}-a_{i j}(t), & \text { if } i \neq j, \\ \sum_{j=1}^{M} a_{i j}(t), & \text { if } i=j,\end{cases}
$$

and $\hat{H}(t)$ is a diagonal matrix of size $M \times M$ with the $i$ th diagonal entry being $h_{i}, i=1,2, \cdots, M$.

Then, following a similar analysis that led to $\dot{Q} \leq 0$, we can show that $\dot{\hat{Q}} \leq 0$. Therefore, we have $\hat{Q}(t) \leq \hat{Q}(T) \leq Q(T) \leq Q_{0}$ for all $t \geq T$.

Obviously, the set of all $(\hat{\tilde{q}}, \hat{\tilde{p}})$ such that $\hat{Q} \leq Q_{0}$, namely,

$$
\Omega:=\left\{\left[\hat{\tilde{q}}^{\mathrm{T}}, \hat{\tilde{p}}^{\mathrm{T}}\right]^{\mathrm{T}} \in R^{2 M n}: \hat{Q}(\hat{\tilde{q}}, \hat{\tilde{p}}) \leq Q_{0}\right\}
$$

is an invariant set. We next show that this is also a compact set. In view of (5.15) and (5.16), $\tilde{p}_{i}^{\mathrm{T}} \tilde{p}_{i} \leq 2 Q_{0}$ for all $i=1,2, \cdots, M$. That is, all $\tilde{p}_{i}$ are bounded, $i=1,2, \cdots, M$. Recall that, at the beginning of this part of proof, we have already established that the distance between any informed agent and the virtual leader is bounded for all $t \geq 0$, and we have also shown that the distance between any Type I uninformed agent and the virtual leader is bounded for all $t \geq 0$. That is, all $\tilde{q}_{i}$ are bounded, $i=1,2, \cdots, M$. This shows that the set $\Omega$ is compact.

In view of $(5.17)$, it is clear that $\dot{\hat{Q}}=0$ if and only if $\hat{\tilde{p}}^{\mathrm{T}}\left(\left(k_{2} \hat{L}(t)+c_{2} \hat{H}(t)\right) \otimes I_{n}\right) \hat{\tilde{p}}=0$. Since both $\hat{L}(t)$ and $\hat{H}(t)$ are positive semi-definite, we have that $\hat{\tilde{p}}^{\mathrm{T}}\left(\hat{L}(t) \otimes I_{n}\right) \hat{\tilde{p}}=0$ and $\hat{\tilde{p}}^{\mathrm{T}}\left(\hat{H}(t) \otimes I_{n}\right) \hat{\tilde{p}}=0$. Let $\hat{G}(t)$ denote 
the neighborhood graph consisting of all the informed agents and Type I uninformed agents. At any time $t \geq T$, suppose that $\hat{G}(t)$ consists of $m(t), 1 \leq m(t) \leq M$, connected subgraphs, and there are $M_{k}(t)$ agents in the $k$ th, $1 \leq k \leq m(t)$, connected subgraph.

Then based on the proof in [22],we can have the following lemma.

Lemma 9 [22] $\hat{\tilde{p}}^{T}\left(\hat{L}(t) \otimes I_{n}\right) \hat{\tilde{p}}=0$ implies that

$$
\hat{\tilde{p}}_{1}^{k}=\hat{\tilde{p}}_{2}^{k}=\cdots=\hat{\tilde{p}}_{M_{k}(t)}^{k}, k=1,2, \cdots, m(t),
$$

namely, the velocity for agents in the same connected subgroup reach consensus. And, $\hat{\tilde{p}}^{T}\left(\hat{H}(t) \otimes I_{n}\right) \hat{\tilde{p}}=0$ implies that $\tilde{p}_{i}=0$ if agent $i$ is informed, $i=1,2, \cdots, M$.

Recall that we have proven that $\dot{\hat{Q}} \leq 0$. It is easy to see that the whole system considered in this chapter is autonomous, since the system dynamics only depend on the initial conditions of agents' states and do not depend on any other independent variables. By the LaSalle Invariance Principle, all trajectories of the agents that start from $\Omega$ will converge to the largest invariant set inside the region

$$
S:=\left\{\left[\hat{\tilde{q}}^{\mathrm{T}}, \hat{\tilde{\tilde{p}}}^{\mathrm{T}}\right]^{\mathrm{T}} \in R^{2 M n}: \dot{\hat{Q}}=0\right\}
$$

Now we will prove that the neighborhood graph will not switch within the invariant set $S$. To prove this, let's assume that the neighborhood graph switches at time $t^{\prime}$, i.e., at time $t^{\prime}$, either an agent $i$ is disconnected from another agent $j$ or an agent $i$ is connected with another agent $j$. In either case, we have $\tilde{p}_{i}\left(t^{\prime}\right) \neq \tilde{p}_{j}\left(t^{\prime}\right)$. It then follows from (5.11), (5.17) and Lemma 9 that $\dot{\hat{Q}}<0$ at least within a small time interval around time $t^{\prime}$, which contradicts with the fact that $\dot{\hat{Q}}=0$ within the invariant set $S$. Therefore, we conclude that the neighborhood graph will not switch within the set $S$.

Now we divide the connected subgroup of agents into two categories. A connected subgroup is called an uninformed connected subgroup if it does not include any informed agent. Otherwise, it is called an informed connected subgroup.

Recall that we have proven that the neighborhood graph will not switch within the set $S$. By definition, all Type I uninformed agents must have a path to one informed agent in the fixed neighborhood graph. Consequently, none of the connected subgroups would be uninformed connected subgroups in such a fixed neighborhood graph.

We will now prove that the position tracking errors and velocity tracking errors of all the agents in an informed connected subgroup converge to zero asymptotically. In view of (5.17) and Lemma 9, we already have that, within the largest invariant set $S$, the velocities of all these agents in an informed connected subgroup 
are the same, and $\tilde{p}_{i}=0$ if agent $i$ is informed. This implies that, within $S$, the velocity tracking errors for both the Type I uninformed agent and the informed agents in the same informed connected subgroup are all 0 .

Next, we will prove that, within $S$, the position tracking errors of all agents in an informed connected subgroup is also 0. Recall that we have proven that the velocity tracking errors of all these agents in an informed connected subgroup are identically zero. Therefore, the derivatives of the velocity tracking errors of these agents are all identically zero. Thus,

$$
\sum_{i=1}^{M_{k}(t)} \tilde{q}_{i}^{\mathrm{T}} \dot{\tilde{p}}_{i}=0,
$$

namely,

$$
-k_{1} \tilde{q}_{M_{k}(t)}^{\mathrm{T}}\left(\hat{L}_{M_{k}(t)}(t) \otimes I_{n}\right) \tilde{q}_{M_{k}(t)}-c_{1} \tilde{q}_{M_{k}(t)}^{\mathrm{T}}\left(\hat{H}_{M_{k}(t)} \otimes I_{n}\right) \tilde{q}_{M_{k}(t)}=0
$$

where, $M_{k}(t)$ is the number of agents in this connected subgroup $k, \hat{L}_{M_{k}(t)}(t)$ is the corresponding Lapalacian matrix associated with this connected subgroup $k$, and $\hat{H}_{M_{k}(t)}$ is the corresponding diagonal matrix with the $i$ th diagonal entry being $h_{i}, i=1,2, \cdots, M_{k}(t)$.

Note that both $\hat{L}_{M_{k}(t)}(t)$ and $\hat{H}_{M_{k}(t)}$ are positive semi-definite. Since these agents formed a connected subgroup and at least one entry of the $\hat{H}_{M_{k}(t)}$ is positive, we have that the matrix $\hat{L}_{M_{k}(t)}(t)+\hat{H}_{M_{k}(t)}$ is positive definite $([14,16])$. This implies that, within $S$, the position tracking errors of all these agents in an informed connected subgroup are also 0. Therefore, we have completed Part iii) of Theorem 5.

Note that, for Part ii) of Theorem 5, where the interaction topology among all the follower agents is jointly connected, by the definitions of joint connectivity and Type I uninformed agent, all the uninformed agents would be Type I. Then, by following the proof for Part iii) of Theorem 5, we can conclude that all the informed and uninformed agents will achieve asymptotic tracking of the virtual leader under our proposed control protocols. Therefore, we have also completed Part ii) of Theorem 5.

This completes the proof of Theorem 5 .

\subsubsection{Control Protocols and the Closed-loop System Performance Analysis with- out Virtual Leader Velocity Information}

Recall that, in Section 5.2.1 of this thesis and in $([9,22])$, both the position and velocity information of the virtual leader are assumed to be given to the informed agents. In this section, we make the assumption that only a portion (some, but not all) of the agents are informed agents who are given the position information of the virtual leader, and none of the agents are provided the velocity information of the virtual leader. We 
propose distributed control protocols and show that the whole group of agents will still be driven to track the virtual leader if the underlying dynamic interaction topology among all the follower agents is jointly connected.

More specifically, we propose the following distributed control protocol for each agent $i, i=1,2, \cdots, N$,

$$
u_{i}=k_{1} \sum_{j \in N_{i}(t)} a_{i j}(t)\left(q_{j}-q_{i}\right)+k_{2} \sum_{j \in N_{i}(t)} a_{i j}(t)\left(p_{j}-p_{i}\right)-h_{i} c_{1}\left(q_{i}-q_{\gamma}\right), k_{1}>0, k_{2}>0, c_{1}>0
$$

where $h_{i}, k_{1}, k_{2}, c_{1}$ and $a_{i j}(t)$ are defined the same as those in Section 5.2.1.

It is easy to see that, in comparison with the control protocols (5.6), the proposed control protocols (5.18) do not contain the term $h_{i} c_{2}\left(p_{i}-p_{\gamma}\right)$, and thus informed follower agents are not assumed to be given the virtual leader velocity information.

Without loss of generality, we assume that the first $M_{0}\left(1 \leq M_{0}<N\right)$ agents are informed agents, namely, $h_{i}=1$ for $i=1,2, \cdots, M_{0}$, and $h_{i}=0$ for $i=M_{0}+1, M_{0}+2, \cdots, N$.

To state the main results of this section, we define a nonnegative energy function $Q(q, p)$ in the same form as in Section 5.2.1.

Then our main results on the information consensus of the group of agents under the proposed control protocols can be stated in the following theorem.

Theorem 6 Consider a multi-agent system of $N$ agents whose dynamics are described by (5.1) and steered by the control protocols specified by (5.18). The dynamics of the virtual leader are described by (5.2). Assume that a portion of the follower agents (some, but not all) are informed agents which can only get access to the position information of the virtual leader. Suppose that the initial energy $Q_{0}:=Q(q(0), p(0))$ is finite. Then the following statements hold:

i) The distance between each informed agent and the virtual leader is not greater than $\sqrt{2 Q_{0} / c_{1}}$ for all $t \geq 0$.

ii) If the dynamic interaction topology among the follower agents is jointly connected, then all the informed and uninformed agents will achieve asymptotic tracking of the virtual leader.

iii) In the case that the interaction topology is not jointly connected, all Type I uninformed agents will still achieve asymptotic tracking of the virtual leader, and all informed agents will achieve bounded tracking of the virtual leader. Furthermore, if the agents operate in one dimensional space (i.e., $q_{i} \in R$, $i=1,2, \cdots, N)$, and if there exists at least one Type I uninformed agent, then all informed agent can actually also achieve asymptotic tracking of the virtual leader. 
Remark 6 For part iii) in Theorem 6, it seems counterintuitive that only bounded tracking errors are ensured for informed agents, while asymptotic tracking behavior are guaranteed for Type I uninformed agents. The key to this phenomenon is the asymptotic tracking behavior results from the interactions between informed agents and Type I uninformed agents (which can be seen in the proof of this theorem below). By definition, any Type I uninformed agents must interact with some informed agents (directly or indirectly), and thus asymptotic tracking behavior can be guaranteed. In contrast, an informed agent (or a group of informed agents) might possibly be isolated from all Type I uninformed agents and not necessarily interact with a Type I uninformed agent, in which case, only bounded tracking errors can be guaranteed for these informed agents in the absence of the virtual leader velocity information.

Remark 7 In Theorem 6, it is assumed that a portion of the follower agents (some, but not all) are informed agents, and thus it does not address the limit case that all the follower agents are informed agents. In such a case, by following a similar analysis procedure as the proof for Part i) of Theorem 6, it is straightforward and easy to prove that all these informed agents will achieve bounded tracking of the virtual leader under the proposed control protocols (5.18).

Remark 8 The previous works ([20,21]) also extended [9] to the case that only a fraction of the follower agents are informed, and their proposed control protocols work for the case that the virtual leader has a time varying velocity. However, in both [20] and [21], in order to drive the follower agents to track the virtual leader, it is required that the undirected interaction topology is connected all the time, while in Theorem 6, only joint connectivity condition is required. In addition, in Theorem 6, we assume that only virtual leader position information but not velocity information is provided to the informed follower agents, while in ([20,21]), the informed agents are assumed to know both the position and velocity information of the virtual leader.

Remark 9 The consensus tracking problems were considered in Theorem 5 and Theorem 6. However, the proposed simple distributed control protocols can be easily adapted to achieve other control objectives such as flocking by only making some small changes. For example, if we simply replace the term $k_{1} \sum_{j \in N_{i}(t)} a_{i j}(t)\left(q_{j}-\right.$ $\left.q_{i}\right)$ in (5.6) and (5.18) with $k_{1} \sum_{j \in N_{i}(t)} \hat{a}_{i j}(t)\left(q_{j}-q_{i}\right)$, then flocking control objective can be achieved and 
collision can be avoided, where,

$$
\hat{a}_{i j}(t)= \begin{cases}0, & \text { if } j=i \text { or }\left\|q_{j}(t)-q_{i}(t)\right\|>r, \\ \frac{r-\left\|q_{j}-q_{i}\right\|}{\delta}, & \text { if } j \neq i \text { and }\left\|q_{j}(t)-q_{i}(t)\right\| \in(r-\delta, r] \\ 1, & \text { if } j \neq i \text { and }\left\|q_{j}(t)-q_{i}(t)\right\| \in\left(r-\delta_{1}, r-\delta\right], \\ 1-\frac{2\left(\delta_{1}-\left\|q_{j}-q_{i}\right\|\right)}{\delta_{1}-\delta_{2}}, & \text { if } j \neq i \text { and }\left\|q_{j}(t)-q_{i}(t)\right\| \in\left(r-\delta_{2}, r-\delta_{1}\right], \\ -\frac{\left(r-\delta_{2}\right)^{2}}{\left\|q_{j}-q_{i}\right\|^{2}}, & \text { if } j \neq i \text { and }\left\|q_{j}(t)-q_{i}(t)\right\| \leq r-\delta_{2},\end{cases}
$$

with $0<\delta \leq \delta_{1} \leq \delta_{2}<r$ being positive constants that can be tuned according to specific applications. The proof will remain quite similar after constructing the corresponding $V_{i j}$ for such $\hat{a}_{i j}(t)$, and thus the details are omitted here for brevity.

Proof of Theorem 6: With the same notation and definitions provided in Section 5.2.1, we will use the same form positive semi-definite energy function $Q(q, p), Q(\tilde{q}, \tilde{p})$ and $\hat{Q}(\hat{\tilde{q}}, \hat{\tilde{p}})$ as defined in (5.7), (5.8), (5.11), (5.12), (5.15) and (5.16) to prove Theorem 6.

By directly following a similar proof of Part i) of Theorem 5, it is straightforward to show that the derivative of $Q$ along the trajectories of the follower agents and the virtual leader is given by,

$$
\begin{aligned}
\dot{Q} & =\frac{1}{2} \sum_{i=1}^{N}\left(\dot{U}_{i}(\tilde{q})+\tilde{p}_{i}^{\mathrm{T}} \dot{\tilde{p}}_{i}\right), \\
& =-k_{2} \tilde{p}^{\mathrm{T}}\left(L(t) \otimes I_{n}\right) \tilde{p},
\end{aligned}
$$

where $L(t)$ is the Lapalacian matrix for the network topology.

Since $L(t)$ is positive semi-definite for an undirected graph, we have $\dot{Q} \leq 0$. This implies that $Q(t)$ is a non-increasing function for all $t \geq 0$ and thus $Q(t) \leq Q(0)$ for all $t \geq 0$. It then follows from (5.11) and (5.12) that $c_{1} \tilde{q}_{i}^{\mathrm{T}} \tilde{q}_{i} \leq 2 Q_{0}$ for any informed agent $i$. Hence the distance between an informed agent $i$ and the virtual leader is not greater than $\sqrt{2 Q_{0} / c_{1}}$ for all $t \geq 0$.

This completes the proof of Part i) of Theorem 6.

So far, we have proven that the distance between an informed agent and the virtual leader is not greater than $\sqrt{2 Q_{0} / c_{1}}$ for all $t \geq 0$, and $Q(t) \leq Q(0)$ for all $t \geq 0$. It follows from (5.11) and (5.12) that $\left\|\left(\tilde{p}_{i}\right)\right\| \leq \sqrt{2 Q(0)}, i=1,2, \cdots, N$. Namely, the velocity difference between any agent and the virtual leader is not greater than $\sqrt{2 Q_{0}}$ for all $t \geq 0$. Then, by definition of Type I uninformed agent, there exists a joint path between a Type I uninformed agent and one informed agent in each finite time interval $\left[t_{i}, t_{i+1}\right)$, $i=0,1,2, \cdots$, with the definition of these time intervals $\left[t_{i}, t_{i+1}\right)$ provided in Definition 8 . Then we can 
easily conclude that the distance between any Type I uninformed agent and the virtual leader is also finite for all $t \geq 0$.

Next, We will prove Part ii) of Theorem 6 as follows. Recall that, by the definition of jointly connected topology and Type I uninformed agents, if the interaction topology among all the follower agents is jointly connected, then all the uninformed follower agents will be Type I uninformed agents.

Obviously, the set of all $(\tilde{q}, \tilde{p})$ such that $Q \leq Q_{0}$, namely,

$$
\Omega:=\left\{\left[\tilde{q}^{\mathrm{T}}, \tilde{p}^{\mathrm{T}}\right]^{\mathrm{T}} \in R^{2 M n}: Q(\tilde{q}, \tilde{p}) \leq Q_{0}\right\}
$$

is an invariant set. Since we have proven that all $\tilde{q}_{i}$ and $\tilde{p}_{i}$ are bounded, $i=1,2, \cdots, N$, the set $\Omega$ is compact.

In view of (5.19), it is clear that $\dot{Q}=0$ if and only if $k_{2} \tilde{p}^{\mathrm{T}}\left(L(t) \otimes I_{n}\right) \tilde{p}=0$. Then based on the proof in [22],we can have the following lemma.

Lemma 10 [22] $\tilde{p}^{T}\left(L(t) \otimes I_{n}\right) \tilde{p}=0$ implies that the velocity for agents in the same connected subgroup reach consensus.

Recall that we have proven that $\dot{Q} \leq 0$. It is easy to see that the whole system considered in this chapter is autonomous. Next, by the LaSalle Invariance Principle, all trajectories of the agents that start from $\Omega$ will converge to the largest invariant set inside the region

$$
S:=\left\{\left[\tilde{q}^{\mathrm{T}}, \tilde{p}^{\mathrm{T}}\right]^{\mathrm{T}} \in R^{2 M n}: \dot{Q}=0\right\}
$$

Now we will prove that the neighborhood graph will not switch within the invariant set $S$. To prove this, let's assume that the neighborhood graph switches at time $t^{\prime}$, i.e., at time $t^{\prime}$, either an agent $i$ is disconnected from another agent $j$ or an agent $i$ is connected with another agent $j$. In either case, we have $\tilde{p}_{i}\left(t^{\prime}\right) \neq \tilde{p}_{j}\left(t^{\prime}\right)$. It then follows from (5.11), (5.14) and Lemma 10 that $\dot{Q}<0$ at least within a small time interval around time $t^{\prime}$, which contradicts with the fact that $\dot{Q}=0$ within the invariant set $S$. Therefore, we conclude that the neighborhood graph will not switch within the set $S$.

Now we divide the connected subgroup of agents into two categories. A connected subgroup is called an uninformed connected subgroup if it does not include any informed agent. Otherwise, it is called an informed connected subgroup.

Recall that we have proven that the neighborhood graph will not switch within the set $S$. By definition, if the topology among all the follower agents is jointly connected, then all the informed and uninformed agents must form a single connected group in such a fixed neighborhood graph. 
We will now prove that the position tracking errors and velocity tracking errors of all the agents in this single connected subgroup converge to zero asymptotically. In view of (5.19) and Lemma 10, we already have that the velocities of all these agents in such a connected group are the same. Then, clearly, the control input (5.18) for an uninformed agent in such a fixed connected group is a constant. It follows directly that the control input for an informed agent in the same connected subgroup must also be a constant. In view of the control protocol (5.18), this implies that the position tracking error for this informed agent is also a constant. This implies that the velocity tracking error for this informed agent is 0 . Since the velocities of all agents in such a fixed connected group are the same, we have that the velocity tracking error for each uninformed agent in the same connected group is also 0 .

Next, we will prove that the position tracking errors of all agents in such a fixed connected group is also 0 . Recall that we have proven that the velocity tracking errors of all agents in this fixed connected group are identically zero. Therefore, the derivatives of the velocity tracking errors of these agents are all identically zero. Then we have,

$$
\sum_{i=1}^{N} \tilde{q}_{i}^{\mathrm{T}} \dot{\tilde{p}}_{i}=0
$$

namely,

$$
-k_{1} \tilde{q}^{\mathrm{T}}\left(L(t) \otimes I_{n}\right) \tilde{q}-c_{1} \tilde{q}^{\mathrm{T}}\left(H(t) \otimes I_{n}\right) \tilde{q}=0,
$$

$i=1,2, \cdots, N$.

Note that both $L(t)$ and $H(t)$ are positive semi-definite. Since these agents form a connected group and at least one entry of the $H(t)$ is positive (i.e., there exist at least one informed follower agent), we have that the matrix $L(t)+H(t)$ is positive definite. This implies that the position tracking errors of all the agents in such a single fixed connected group also approach 0 . Therefore, we have completed the proof of Part ii) of Theorem 6.

Finally, we will prove Part iii) of Theorem 6. By directly following the proof of Part iii) of Theorem 5, it is easy to establish that there exists a $T>0$, such that all the informed agents and Type I uninformed agents cannot be influenced by a Type II uninformed agents directly or indirectly for all $t \geq T$. Next, for any $t \geq T$, it is also straightforward to show that $\hat{Q} \leq Q$ and the derivative of $\hat{Q}$ along the trajectories of the follower agents and the virtual leader is given by

$$
\begin{aligned}
\dot{\hat{Q}} & =\frac{1}{2} \sum_{i=1}^{M}\left(\dot{\hat{U}}_{i}(\hat{\tilde{q}})+\tilde{p}_{i}^{\mathrm{T}} \dot{\tilde{p}}_{i}\right), \\
& =-k_{2} \hat{\tilde{\hat{p}}}^{\mathrm{T}}\left(\hat{L}(t) \otimes I_{n}\right) \hat{\tilde{p}}
\end{aligned}
$$


where $\hat{L}(t)=\left[\hat{l}_{i j}(t)\right]_{M \times M}$ is the corresponding Lapalacian matrix of the subgraph consisting of only informed agents and Type I uninformed agents. Since $\hat{L}(t)$ is positive semidefinite for a undirected graph, $\dot{\hat{Q}} \leq 0$, we have $\hat{Q}(t) \leq \hat{Q}(T) \leq Q(T) \leq Q_{0}$ for all $t \geq T$.

Next, following a similar analysis as in the proof of Theorem 5, it is straightforward to show that:

i) The set $\Omega^{\prime}:=\left\{\left[\hat{\tilde{q}}^{\mathrm{T}}, \hat{\tilde{p}}^{\mathrm{T}}\right]^{\mathrm{T}} \in R^{2 M n}: \hat{Q}(\hat{\tilde{q}}, \hat{\tilde{p}}) \leq Q_{0}\right\}$ is invariant and compact;

ii) All trajectories of the agents that start from $\Omega^{\prime}$ will converge to the largest invariant set $S^{\prime}$ inside the region $\Omega_{1}^{\prime}:=\left\{\left[\hat{\tilde{q}}^{\mathrm{T}}, \hat{\tilde{p}}^{\mathrm{T}}\right]^{\mathrm{T}} \in R^{2 M n}: \dot{\hat{Q}}=0\right\}$

iii) The neighborhood graph will not switch within the invariant set $S^{\prime}$.

Now we divide the connected subgroup of agents into 3 categories as follows. A connected subgroup is called an uninformed connected subgroup if it does not include any informed agent. A connected subgroup is called Type A informed connected subgroup if it includes at least one informed agent and at least one Type I uninformed agent. A connected subgroup is called Type B informed connected subgroup if it includes only informed agents.

Recall that we have proven that the neighborhood graph will not switch within the invariant set $S^{\prime}$. By definition, all Type I uninformed agents must have a path to one informed agent in the fixed neighborhood graph within the invariant set $S^{\prime}$. Consequently, none of the connected subgroups would be uninformed connected subgroups in such a fixed neighborhood graph.

For agents in a Type A informed connected subgroup, by simply following the procedure as in the proof for Part ii) of Theorem 6 above, it is straightforward to show that both the position tracking errors and the velocity tracking errors of all the agents in a Type A informed connected subgroup converge to zero asymptotically. In fact, in Part ii) of Theorem 6, all the informed and uninformed agents just finally form a single Type A informed connected group within the set of $S$. Note that, by definition, within the invariant set $S^{\prime}$, each Type I uninformed agent must belong to a Type A informed connected subgroup. Therefore, we have proven that all the Type I uninformed agents will achieve asymptotic tracking of the virtual leader.

Next, we will analyze the position tracking errors and velocity tracking errors of agents in a Type B informed connected subgroup, and prove that all informed agents will achieve bounded tracking of the virtual leader, and will even achieve asymptotic tracking if the agents operate in one dimensional space and there exists at least one Type I uninformed agent.

Recall that, within the largest invariant set $S^{\prime}$, the velocity of all agents in a connected subgroup are the same. In view of the control protocol (5.18), it is easy to see that the agent dynamics equation for an agent in such a Type B informed connected subgroup reduces to the following form, 


$$
\left\{\begin{array}{l}
\dot{\tilde{q}}_{i}=\tilde{p}_{i} \\
\dot{\tilde{p}}_{i}=\delta_{i}-c_{1} \tilde{q}_{i}
\end{array}\right.
$$

where $\delta_{i}=\sum_{j \in N_{i}(t)} a_{i j}\left(\tilde{q}_{j}-\tilde{q}_{i}\right)$ is independent of $t$ since $\tilde{p}_{i}$ are identically the same for all agents in this connected subgroup. Note that, within the largest invariant set $S^{\prime}$, the topology does not switch, and it follows from (5.21) that the agents' dynamics are decoupled on the $n$ dimensions. Therefore, (5.21) can be rewritten as

$$
\dot{x}_{i l}=A x_{i l}+B v, l=1,2, \cdots, n,
$$

with $x_{i l}=\left[\begin{array}{ll}\tilde{q}_{i l} & \tilde{p}_{i l}\end{array}\right]^{\mathrm{T}}, A=\left[\begin{array}{ll}01 ;-c_{1} 0\end{array}\right], B=\left[\begin{array}{ll}0 & \delta_{i l}\end{array}\right]^{\mathrm{T}}$ and $v=1$. The solution of (5.22) can be written as $x_{i l}(t)=e^{A t} x_{i l}(0)+\int_{0}^{t} e^{A(t-\tau)} B v(\tau) d \tau, l=1,2, \cdots, n$. Note that, for $A=\left[01 ;-c_{1} 0\right]$ with $c_{1}>0$, it is easy to compute that

$$
e^{A t}=\left[\begin{array}{cc}
\cos \left(\sqrt{c_{1}} t\right) & \sin \left(\sqrt{c_{1}} t\right) \\
-\sin \left(\sqrt{c_{1}} t\right) & \cos \left(\sqrt{c_{1}} t\right)
\end{array}\right]
$$

and

$$
e^{A t} x_{i l}(0)=\left[\begin{array}{c}
\tilde{q}_{i l}(0) \cos \left(\sqrt{c_{1}} t\right)+\tilde{p}_{i l}(0) \sin \left(\sqrt{c_{1}} t\right) \\
-\tilde{q}_{i l}(0) \sin \left(\sqrt{c_{1}} t\right)+\tilde{p}_{i l}(0) \cos \left(\sqrt{c_{1}} t\right)
\end{array}\right]
$$

Recall that, within the largest invariant set $S^{\prime}$, for these agents in the same connected subgroup, their velocities are always the same. Then it follows directly from (5.23) that, within the largest invariant set $S^{\prime}$, the position tracking errors $\tilde{q}_{i}$ of all these agents in the same Type B informed connected group would also be identically the same. Therefore, all the $\delta_{i}$ here would be 0 , and $x_{i l}(t)=e^{A t} x_{i l}(0), l=1,2, \cdots, n$. In view of (5.23), it is easy to observe that, on each dimension $l, l=1,2, \cdots, n$, the position tracking error trajectory $\tilde{q}_{i l}(t)$ is a sinusoidal function fluctuating around zero, and the velocity tracking error trajectory $\tilde{p}_{i l}(t)$ is also a sinusoidal function fluctuating around zero. Recall that, within the largest invariant set $S^{\prime}$, for an informed agent that belongs to a Type A informed connected subgroup, the position tracking error and the velocity tracking error are both zero. Therefore, we have proven that, for all informed agents, the position tracking errors and velocity tracking errors are bounded.

Next, we will prove that, if the agents operate in one dimensional space (i.e., $n=1$ ) and if there is at least one Type I uninformed follower agent, the position tracking errors and velocity tracking errors of all informed agents approach zero asymptotically. The detail of the proof is presented below.

If there is at least one Type I uninformed follower agent, then there exists at least one Type A informed connected subgroup in the fixed neighborhood graph. Note that we have proven that the position tracking errors of all agents in a Type A informed connected subgroup converge to zero asymptotically. We have 
also proven that, for a Type B informed connected subgroup, the position tracking error trajectories $\tilde{q}_{i}(t)$ is a sinusoidal function fluctuating around zero. Then at some time point this Type B informed connected subgroup will be connected with the Type A informed connected subgroup, which implies a neighborhood graph switching occurs. Since we have proven that the neighborhood graph will not switch within the largest invariant set $S^{\prime}$, this is a contradiction. Therefore, we can conclude that, if there is at least one Type I uninformed follower agent, then none of the connected subgroup in the final fixed neighborhood graph would be Type B informed connected subgroup (i.e., all the connected subgroups in the final fixed neighborhood graph would be Type A informed connected subgroup). Recall that we have already proven that, for all agents in a Type A informed connected subgroup, both the position tracking errors and the velocity tracking errors converge to zero asymptotically. Therefore, we have completed the proof of Part iii) of Theorem 6 .

This completes the proof of Theorem 6 .

\subsubsection{Connectivity Enhancing Coordinated Tracking Control Protocols and the Closed-loop System Performance Analysis}

In this section, we propose a new connectivity enhancing mechanism, and analyze the closed-loop performance of such connectivity enhancing coordinated tracking control protocols. Existing connectivity preserving coordinated tracking control protocols all assume the interaction topology to be initially connected, and technical issues associated with those edges that are not initially existent have not been adequately addressed. In fact, in many existing connectivity preserving control works, for those edges that were not initially existent, their weights are usually simply treated as $a_{i j}(t)=1$ for $j \in N_{i}(t)$ and $a_{i j}(t)=0$ for $j \notin N_{i}(t)$, sometimes enhanced with a hysteresis nonlinearity. As a result, those newly formed topology edges that were not initially existent are not guaranteed to be maintained, and an initial connected topology is required. In addition, such a sudden jump of $a_{i j}(t)$ will lead to the discontinuity on the right side of the agent dynamics equations. Because of such discontinuity resulting from jumps in $a_{i j}(t)$, the standard assumption (i.e., essentially one sided local Lipschitz condition) to guarantee solution uniqueness will not be satisfied, even in the sense of Filippov solution. In contrast, our proposed topology connectivity enhancing mechanism is effective in maintaining both the initially existent topology edges and the newly formed topology edges, which enables us to relax the more restrictive requirement that the interaction topology is initially connected. Also, our proposed algorithms are designed in a way such that the local Lipschitz condition is satisfied and thus technical issues on solution uniqueness are avoided. 
To be specific, we propose the following distributed connectivity enhancing coordinated tracking control protocols for each agent $i, i=1,2, \cdots, N$,

$$
u_{i}=k_{1} \sum_{j \in N_{i}(t)} b_{i j}(t)\left(q_{j}-q_{i}\right)+k_{2} \sum_{j \in N_{i}(t)} a_{i j}(t)\left(p_{j}-p_{i}\right)-h_{i} c_{1}\left(q_{i}-q_{\gamma}\right), k_{1}>0, k_{2}>0, c_{1}>0
$$

where $h_{i}, k_{1}, k_{2}, c_{1}$ and $a_{i j}(t)$ are defined the same as those in Section 5.2.2, and $b_{i j}(t)$ is a locally Lipschitz function of $q_{j}(t)-q_{i}(t)$, defined for connectivity enhancing purpose as follows,

i) If $\left\|q_{i}(0)-q_{j}(0)\right\|<r$,

$$
b_{i j}(t)= \begin{cases}\frac{\delta^{2}}{\left(\left\|q_{i}-q_{j}\right\|-r\right)^{2}}, & \text { if }\left\|q_{j}(t)-q_{i}(t)\right\| \in(r-\delta, r) \\ 1, & \text { if }\left\|q_{j}(t)-q_{i}(t)\right\| \leq r-\delta .\end{cases}
$$

ii) If $\left\|q_{i}(0)-q_{j}(0)\right\| \geq r$, let $t_{\text {connect }_{i j}}$ be the first time instant that $\|\left(q_{i}(t)-q_{j}(t) \| \leq r-\delta\right.$, where $\delta \in(0, r)$ is a constant parameter which can be adjusted according to specific applications, then 1 ) for $t \leq t_{\text {connect }_{i j}}$, $b_{i j}(t)=a_{i j}(t) ;$ and 2$)$ for $t \geq t_{\text {connect } i j}$,

$$
b_{i j}(t)= \begin{cases}\frac{\delta^{2}}{\left(\left\|q_{i}-q_{j}\right\|-r\right)^{2}}, & \text { if }\left\|q_{j}(t)-q_{i}(t)\right\| \in(r-\delta, r) \\ 1, & \text { if }\left\|q_{j}(t)-q_{i}(t)\right\| \leq r-\delta .\end{cases}
$$

To state the main results of this section, we define a nonnegative energy function $Q(q, p)$ as follows,

$$
Q(q, p)=\frac{1}{2} \sum_{i=1}^{N}\left(U_{i}(q)+\left(p_{i}-p_{\gamma}\right)^{\mathrm{T}}\left(p_{i}-p_{\gamma}\right)\right),
$$

where

$$
U_{i}(q)=\frac{k_{1}}{2} \sum_{j=1, j \neq i}^{N} V_{i j}\left(q_{i}, q_{j}\right)+h_{i} c_{1}\left(q_{i}-q_{\gamma}\right)^{\mathrm{T}}\left(q_{i}-q_{\gamma}\right) .
$$

with $V_{i j}\left(q_{i}, q_{j}\right)$ being a differentiable function whose derivative satisfies that

$$
\dot{V}_{i}=\sum_{j \in N_{i}(t)} b_{i j}(t)\left(\tilde{q}_{j}-\tilde{q}_{i}\right)^{\mathrm{T}}\left(\tilde{p}_{j}-\tilde{p}_{i}\right)
$$

where $V_{i}=\frac{1}{2} \sum_{j=1, j \neq i}^{N} V_{i j}$. 
Given the expression of $b_{i j}(t)$, it is not difficult to construct such $V_{i j}$. By the definition of $b_{i j}(t)$, if $\left\|q_{i}(0)-q_{j}(0)\right\| \geq r$ and if the expression of $b_{i j}(t)$ switches at $t=t_{\text {connect }_{i j}}$, the expression of $V_{i j}$ will also switch at $t=t_{\text {connect } i j_{i j}}$. Note that $V_{i j}$ are chosen such that it is continuous at such switching time instant $t=t_{\text {connect }_{i j}}$.

Then our main results on the information consensus of the group of agents under the proposed control protocols can be stated in the following theorem.

Theorem 7 Consider a multi-agent system of $N$ agents whose dynamics are described by (5.1) and steered by the control protocols specified by (5.24). The dynamics of the virtual leader are described by (5.2). Assume that a portion of the follower agents (some, but not all) are informed agents which can only get access to the position information of the virtual leader. Suppose that the initial energy $Q_{0}:=Q(q(0), p(0))$ is finite. Then the following statements hold:

i) The distance between each informed agent and the virtual leader is not greater than $\sqrt{2 Q_{0} / c_{1}}$ for all $t \geq 0$.

ii) If the dynamic interaction topology among the follower agents is jointly connected, then all the informed and uninformed agents will achieve asymptotic tracking of the virtual leader.

iii) In the case that the interaction topology is not jointly connected, all Type I uninformed agents will still achieve asymptotic tracking of the virtual leader, and all informed agents will achieve bounded tracking of the virtual leader. Furthermore, if the agents operate in one dimensional space (i.e., $q_{i} \in R$, $i=1,2, \cdots, N)$, and if there exists at least one Type I uninformed agent, then all informed agents will also achieve asymptotic tracking of the virtual leader.

iv) All those initially existent edges of the topology graph will be preserved, and any new edge $e_{i j}$ that is not initially existent can also be maintained for all $t \geq t_{\text {connect } i j}$ if the distance between agent $i$ and $j$ is not greater than $r-\delta$ at certain time instant $t_{\text {connect }_{i j}}>0$.

Proof of Theorem 7: To prove Theorem 7, the switched energy function $Q$ defined above in (5.25) is chosen as the Lyapunov function. Note that, although the expression of $Q$ switches at time instants when the expression of some $b_{i j}$ switches, the above constructed $Q$ is still continuous and differentiable except at the time of switching.

In particular, by directly following a similar proof as Part i) of Theorem 5, for all those time intervals during which $Q$ does not switch, it is straightforward to show that the derivative of $Q$ along the trajectories 
of the follower agents and the virtual leader is given by,

$$
\begin{aligned}
\dot{Q} & =\frac{1}{2} \sum_{i=1}^{N}\left(\dot{U}_{i}(\tilde{q})+\tilde{p}_{i}^{\mathrm{T}} \dot{\tilde{p}}_{i}\right) \\
& =-k_{2} \tilde{p}^{\mathrm{T}}\left(L(t) \otimes I_{n}\right) \tilde{p}
\end{aligned}
$$

where $L(t)$ is the Lapalacian matrix as defined in Section 3.4. Therefore, we have that $\dot{Q} \leq 0$ when $Q$ does not switch. Recall that $Q$ is continuous at the time of switching. It follows directly that $Q(t) \leq Q(0)$ for all $t \geq 0$. From this, it is easy to obtain Part i) of Theorem 7 .

Since we have prove that $Q(t) \leq Q(0)$ for all $t \geq 0$, it follows directly that all those initially existent edges of the topology graph will be preserved, and any new edge $e_{i j}$ that is not initially existent can also be maintained for all $t \geq t_{\text {connect }_{i j}}$ if the distance between agent $i$ and $j$ is not greater than $r-\delta$ at certain time instant $t_{\text {connect }_{i j}}>0$. Otherwise, $V_{i j}$ (and thus $Q(t)$ ) will increase to infinity, which is a contradiction. Therefore, we have also proven Part iv) of Theorem 7.

Next, based on the proven Part iv) of Theorem 7 and by definition of $b_{i j}(t)$, it is easy to see that $V_{i j}$ can only switch at most once for a pair of agent $i$ and $j$. Therefore, the constructed switched Lyapunov function $Q$ can at most switch finite times. As a result, the switched Lyapunov function $Q$ chosen here is a very special case of the multiple Lyapunov functional approach where the values of the Lyapunov functions coincide at the time of switching, and the total number of switching is finite. The analysis of such a switched Lyapunov function $Q$ can simply follow the same analysis procedure as with a common Lyapunov function in Section 5.2.2. As a result, it is not hard to obtain the results in Part ii) and Part iii) of Theorem 7 , by mimicking the proof procedure of Theorem 6 using the switched Lyapunov function $Q$ constructed above for Theorem 7. The proof details are omitted for brevity.

This completes the proof of Theorem 7.

Remark 10 From Theorem 7, since the proposed new topology connectivity maintenance mechanism is effective in maintaining both the initially existent edges and the new edges that are not initially existent, it is obvious that the connectivity enhancing coordinated tracking objective can still be achieved if the network topology graph is not initially connected, but only connected in an integral sense. As a result, such a more effective connectivity maintenance mechanism will make the mild network connectivity condition to be even more easily satisfied in real world implementations. Different from the existing connectivity preserving control protocols, our proposed algorithms are designed in a way such that the local Lipschitz condition is satisfied and thus technical issues on solution uniqueness of the closed-loop system are avoided. This proposed mechanism 
would be useful especially for large scale network applications where an initially connected configuration is infeasible or inefficient. The constant parameter $\delta$ here could be adjusted according to specific applications.

Remark 11 In Theorem 7, the proposed topology connectivity enhancing mechanism is applied to Section 5.2.2 for the situation where the virtual leader velocity information is not available. But it is easy to see that such a topology connectivity enhancing mechanism is also applicable to the situation in Section 5.2.1, where the virtual leader velocity information is available. In fact, such a proposed topology connectivity enhancing mechanism and the corresponding constructed switched energy function (Lyapunov function) proof technique can be applied to relax the initial topology connectivity condition in many other connectivity preserving coordinated control problems of multi-agent systems.

\subsection{Simulation Results}

\subsubsection{Distributed Coordinated Tracking Control with Virtual Leader Velocity Information}

We consider a network of 39 follower agents and a virtual leader moving in a 2-dimensional space. The initial position and velocity of the virtual leader are set as $q_{\gamma}(0)=[5,5]^{\mathrm{T}}$ and $p_{\gamma}(0)=[0.1,0.1]^{\mathrm{T}}$. The initial positions and initial velocities of the 39 follower agents are chosen randomly from the boxes $[-20,20] \times[-20,20]$ and $[-0.1,0.1] \times[-0.1,0.1]$, respectively. The parameters in the control protocols $(5.6)$ are set as $k_{1}=1.5$, $k_{2}=3, c_{1}=5, c_{2}=2$, and $\delta=0.01$. The influencing/sensing radius is chosen as $r=10$. The number of informed agents is 1 .

Shown in Figs. 5.1 and 5.2 are some simulation results. In these figures, the green solid line denotes the informed follower agent and blue dotted lines denote the uninformed follower agents. Note that, for the chosen initial conditions, by analyzing the eigenvalues of the Laplacian matrix of the initial interaction topology at $t=0$, we verify that zero is not a simple eigenvalue (in fact, it has multiplicity of two) of the initial Laplacian matrix and thus the initial interaction topology among all the follower agents is not connected. In fact, this can also be observed by looking at the position tracking errors in Figs. 5.1 and 5.2, from which we can get a rough idea that there exist two separate connected subgroups at $t=0$ (recall that the sensing radius $r=10$ ). Obviously, in such a case, the assumption that the interaction topology among all the follower agents are connected all the time, which is a quite standard assumption in the literature for this type of problems (e.g., ( $[20,21]))$, is not satisfied. Our Theorem 5 establishes that, even though the dynamic interaction topology among all the follower agents is not connected all the time, under our proposed control protocols, all the informed and uninformed agents are still guaranteed to achieve asymptotic tracking 
of the virtual leader as long as the milder joint connectivity assumption is satisfied. This is verified by the simulation results in Figs. 5.1 and 5.2, from which we can see all the informed and uninformed follower agents are driven to track the virtual leader, and both the position tracking errors and velocity tracking errors converge to zero asymptotically, even if the interaction topology is not connected all the time. Such simulation results demonstrate that our Theorem 5 enriches the literature on this type of problems by relaxing the more restrictive condition on network topology connectivity (i.e., the topology is connected all the time) to a milder joint connectivity condition.

On the other hand, there are situations where even such a mild joint connectivity condition is not necessary guaranteed to be satisfied. In such cases, the new network connectivity enhancing mechanisms we propose in this chapter can be used to help achieve the desired tracking performance. We next conduct simulation study to verify the performance of the control protocols (5.6) with the topology connectivity enhancing mechanism implemented. For example, Fig. 5.3 shows the simulation results for comparison of the tracking performance under our proposed control protocols (5.6) without and with the network topology connectivity enhancing mechanism. For the simulation in Fig. 5.3, the initial positions and initial velocities of the 39 follower agents are chosen randomly from the boxes $[-20,20] \times[-20,20]$ and $[-15,15] \times[-15,15]$, respectively. Parameter $\delta$ is set to be 1 . All other control parameters as well as the initial virtual leader position and velocity are the same as those used for Figs. 5.1 and 5.2. As can be seen by comparing Fig. 5.3 and Fig. 5.4, for the chosen initial conditions, without the topology connectivity enhancing mechanism, even though the initial interaction topology is connected, the dynamic interaction topology among all the follower agents does not satisfy the mild joint connectivity condition, and as a result, only the informed follower agents and a portion of the uninformed agents (i.e., Type I uninformed follower agents) achieve asymptotic tracking of the virtual leader. In contrast, with the help of the topology connectivity enhancing mechanism, the dynamic interaction topology among all the follower agents remains connected, and all the informed and uninformed agents achieve asymptotic tracking of the virtual leader. In fact, our extensive simulation study verify that, with the help of the proposed topology connectivity enhancing mechanism, if the initial interaction topology among all the follower agents is connected, then it is always the case that all the informed and uninformed agents will track the virtual leader asymptotically. Moreover, under our proposed topology connectivity enhancing mechanism, for situations where the topology is not initially connected, when two initially separated connected subgroups interact with each other with the closest distance between the two groups being less than $r-\delta$ at some time instant, they will be combined into a single connected group and the connectivity would be maintained afterwards. As a result, the network topology connectivity could be enhanced as time goes by. This indicates that our proposed connectivity enhancing mechanism is effective in helping enhance the topology connectivity and cause the informed and uninformed agents to track the virtual leader. 

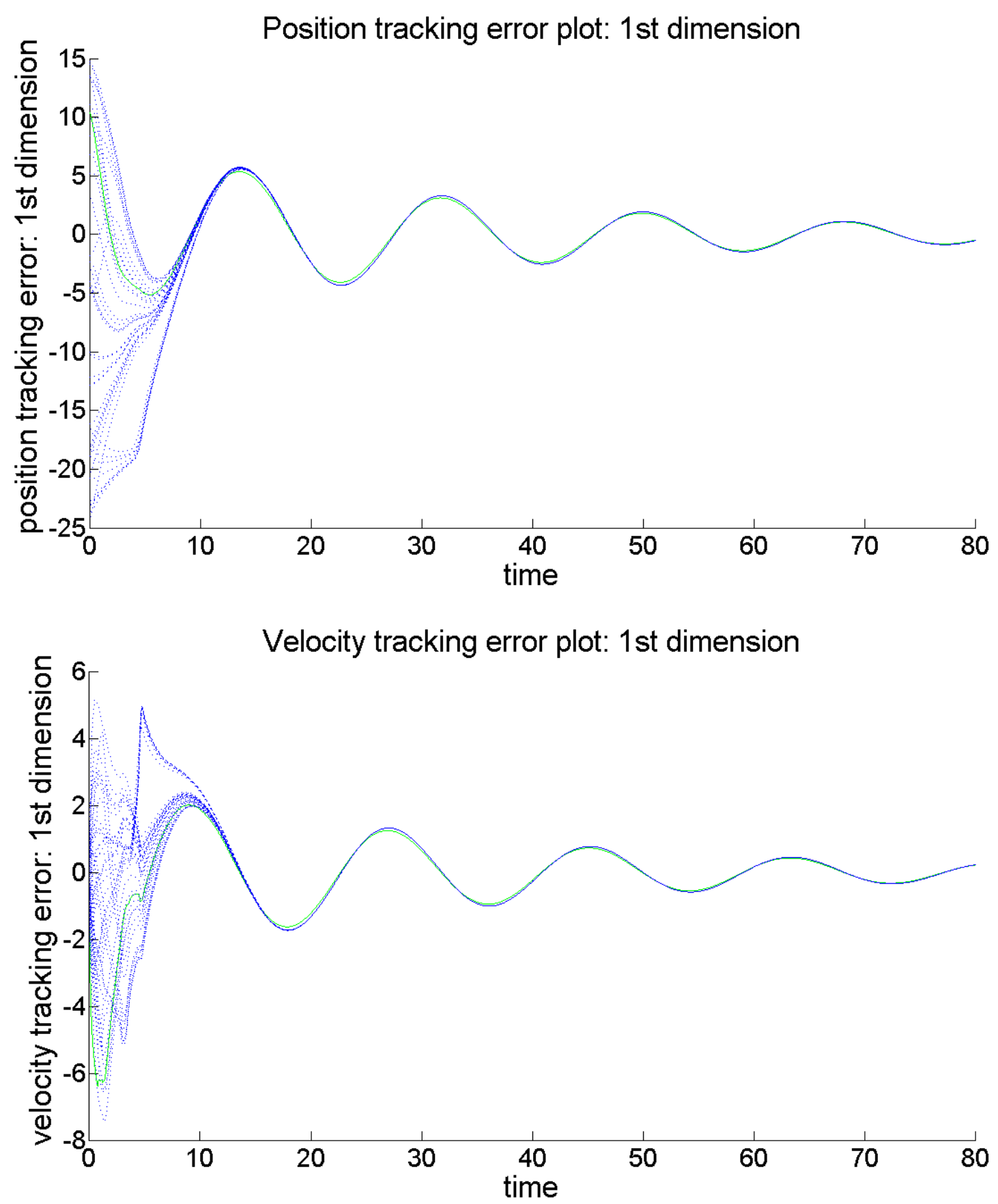

Figure 5.1: The 1st dimension tracking errors between follower agents and the virtual leader under control protocols (5.6)

\subsubsection{Distributed Coordinated Tracking Control without Virtual Leader Veloc- ity Information}

In this section, we investigate the performance of our proposed control protocols (5.18) without the virtual leader velocity information. In particular, we again consider a network of 39 follower agents and a virtual 

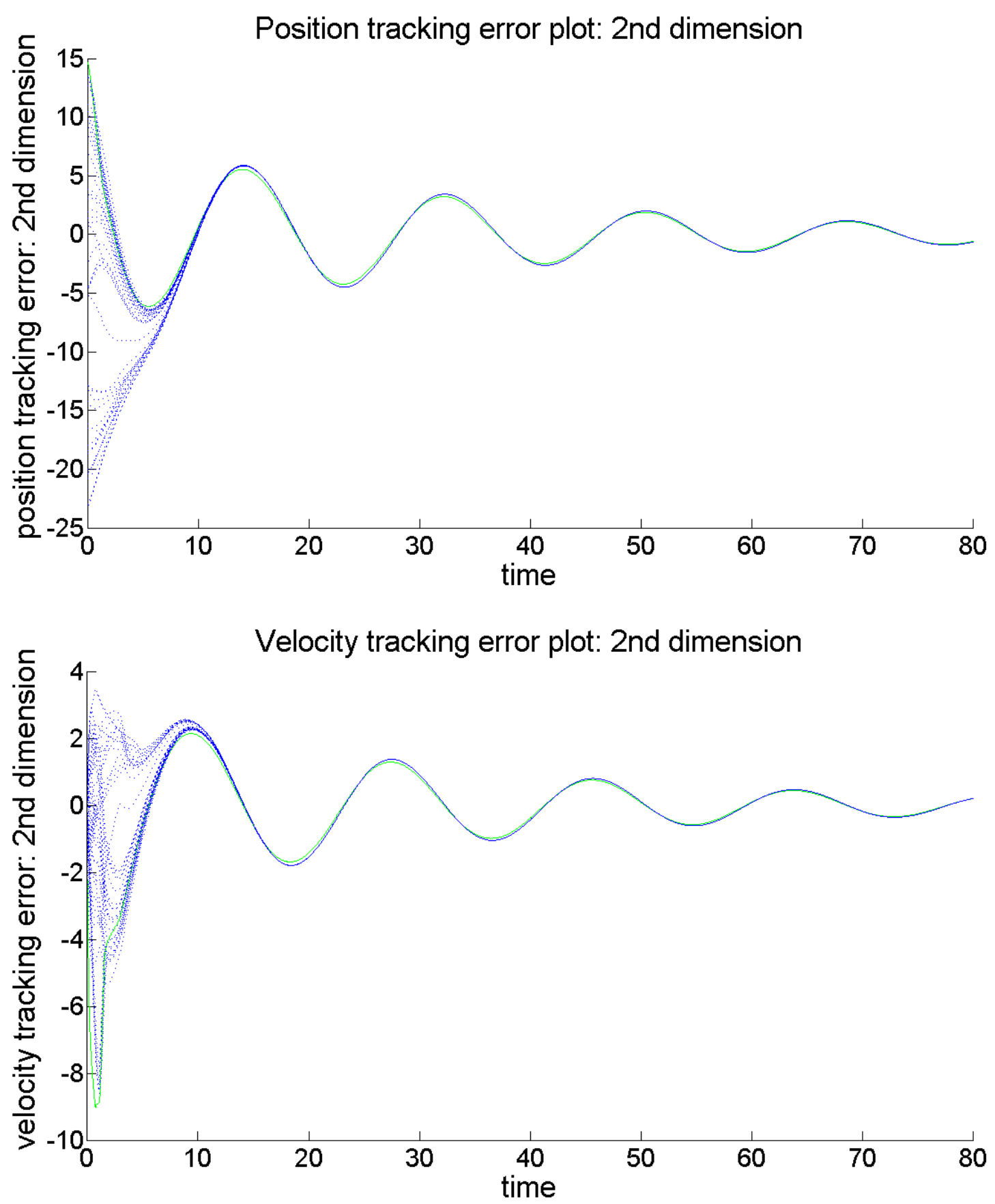

Figure 5.2: The 2nd dimension tracking errors between follower agents and the virtual leader under control protocols (5.6)

leader moving in a 2-dimensional space. The initial position and velocity of the virtual leader are set as $q_{\gamma}(0)=[5,5]^{\mathrm{T}}$ and $p_{\gamma}(0)=[0.1,0.1]^{\mathrm{T}}$. The initial positions and initial velocities of the 39 follower agents were chosen randomly from the boxes $[-20,20] \times[-20,20]$ and $[-0.1,0.1] \times[-0.1,0.1]$, respectively. The parameters in the control protocol (5.6) are set as $k_{1}=1.5, k_{2}=3, c_{1}=5$ and $\delta=0.01$. The influencing/sensing radius is chosen as $r=10$. The number of informed agents is 1 . 

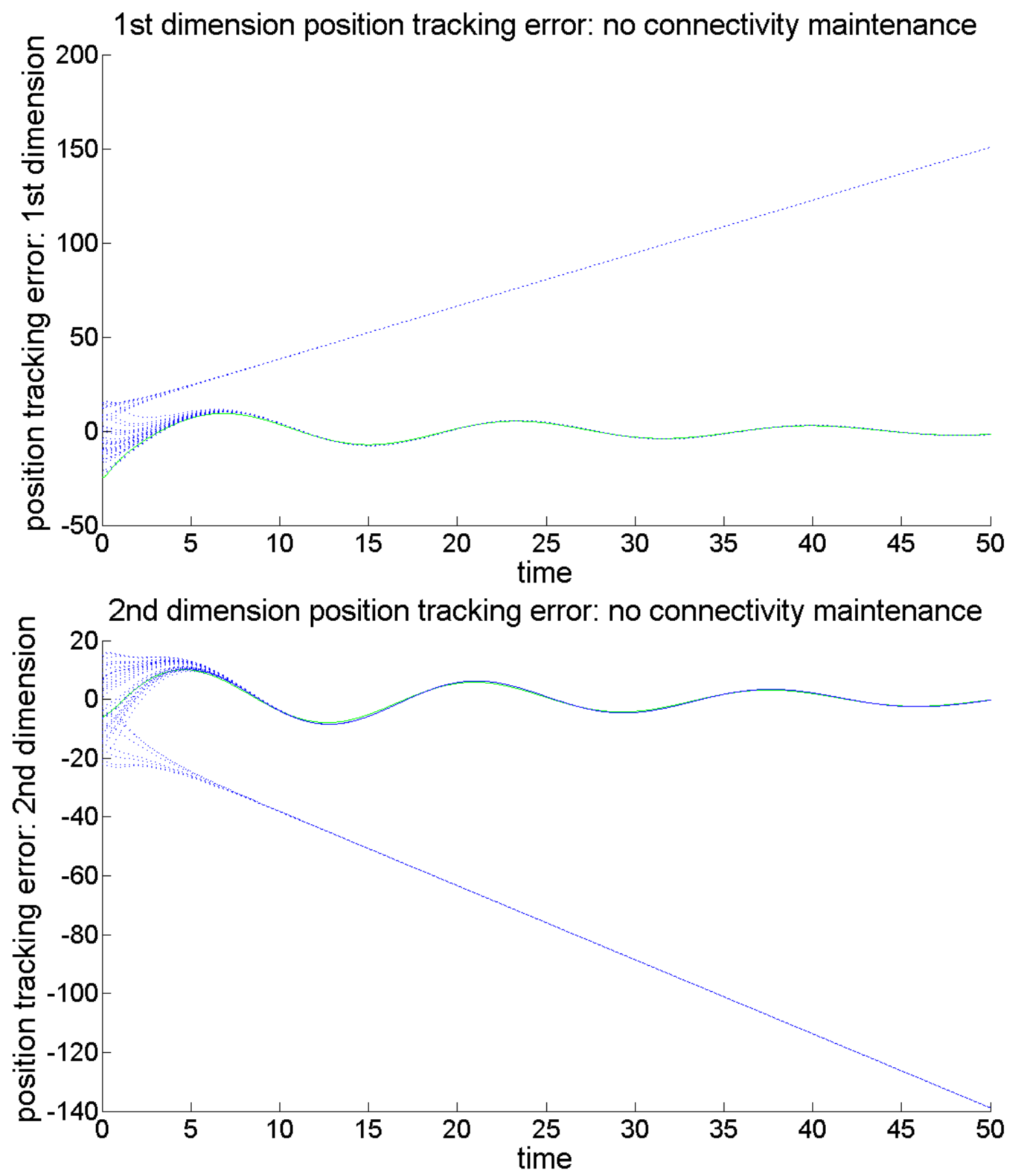

Figure 5.3: Tracking performance under control protocols (5.6) without the proposed topology connectivity maintenance mechanism

Shown in Figs. 5.5 and 5.6 are some simulation results. For the simulation results in Figs. 5.5 and 5.6, we have used exactly the same initial condition for Figs. 5.1 and 5.2 as in Section 5.3.1, and the initial interaction topology is not connected. Again, in these figures, the green solid line denotes the informed follower agent and blue dotted lines denote the uninformed follower agents. As can be seen in Figs. 5.5 and 5.6 , in the absence of the virtual leader velocity information, our proposed control protocols are still able to 

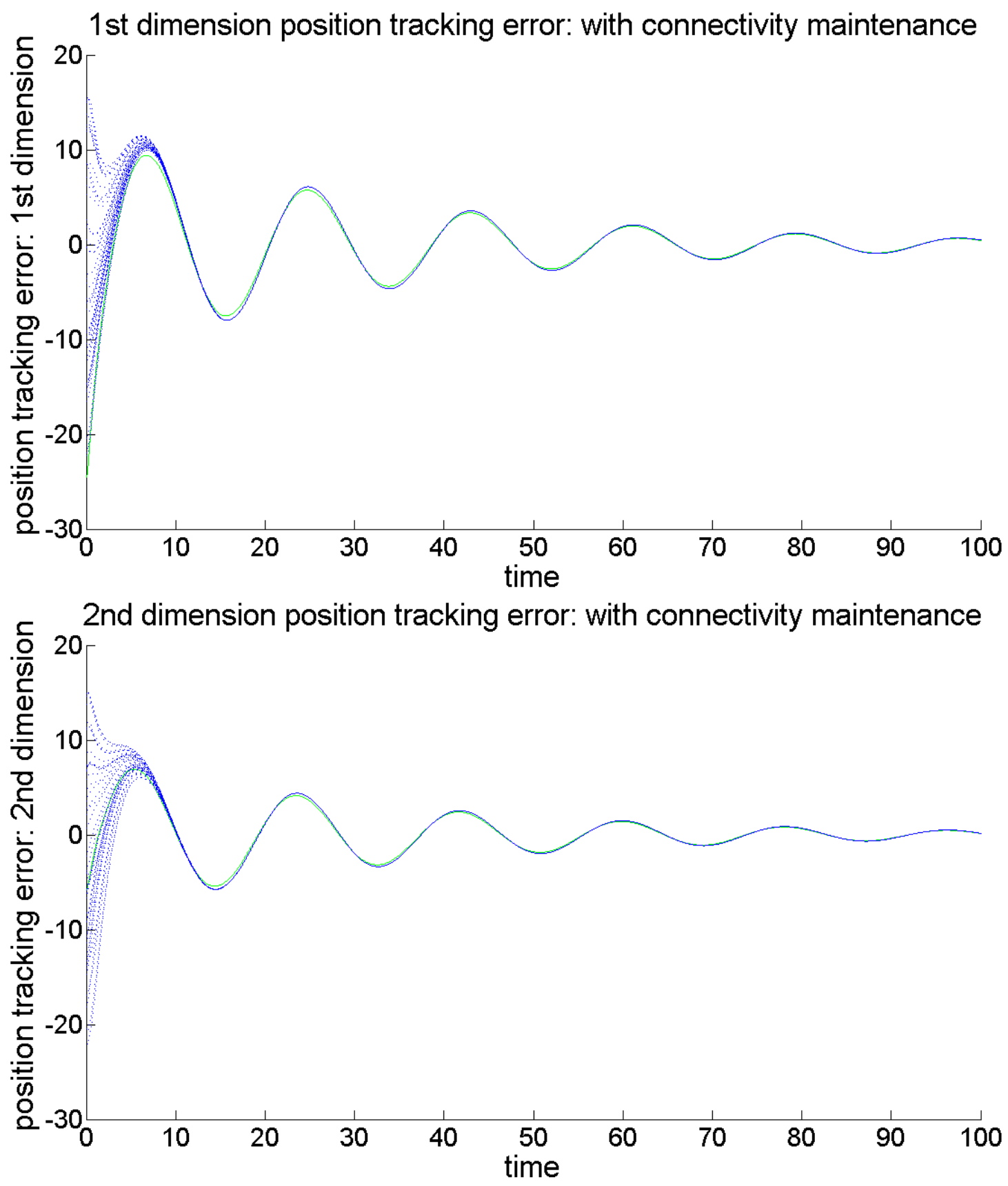

Figure 5.4: Tracking performance under control protocols (5.6) with the proposed topology connectivity maintenance mechanism

drive all the informed and uninformed agents to achieve asymptotic tracking of the virtual leader, even if the underlying interaction topology is only jointly connected but not connected all the time. Such simulation results demonstrate that our Theorem 6 enriches the literature on this type of problems by not only relaxing the more restrictive condition on network topology connectivity (i.e., the topology is connected all the time), but also relaxing the requirement on the availability of the virtual leader velocity information. 

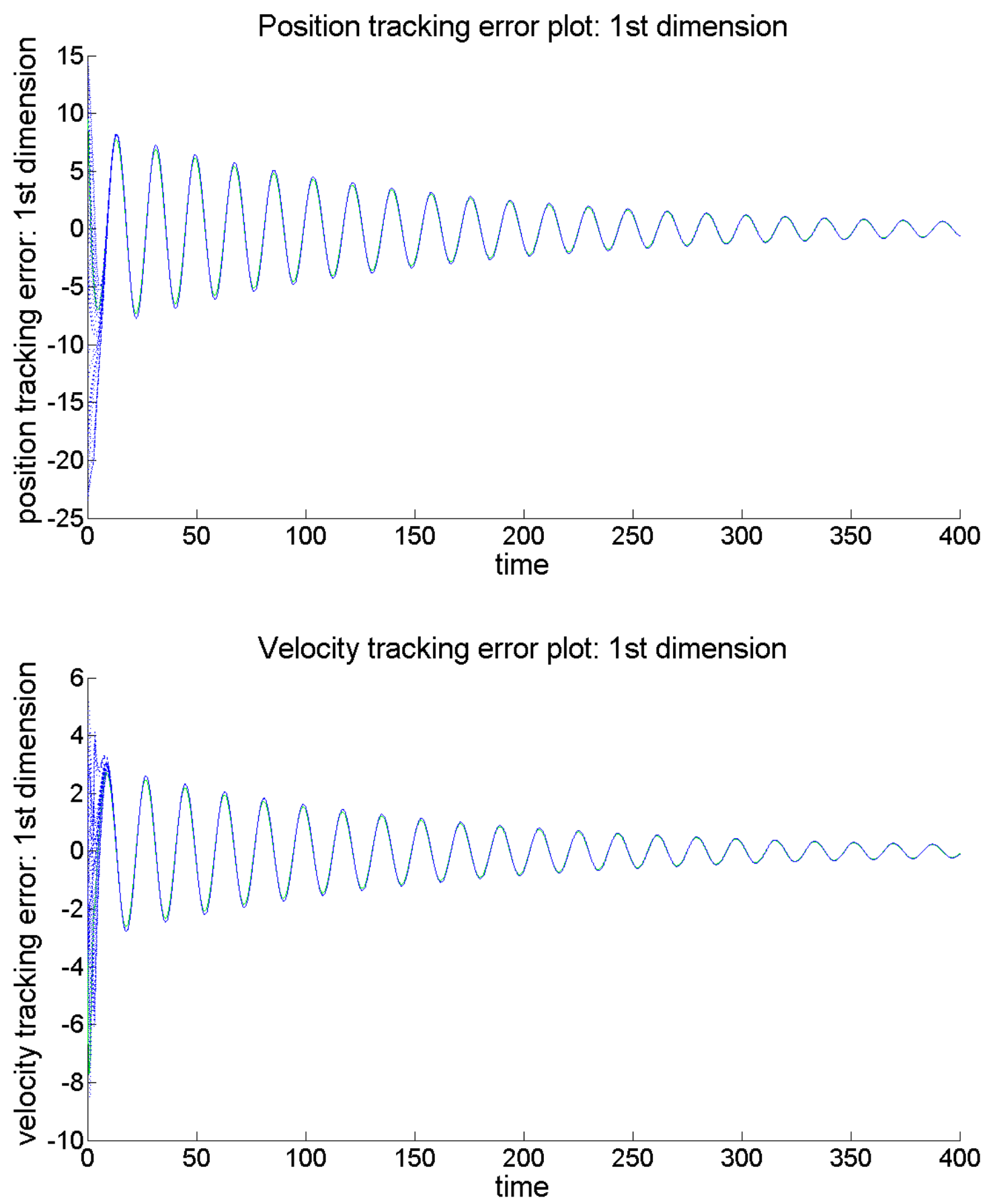

Figure 5.5: The 1st dimension tracking errors between follower agents and the virtual leader under control protocols (5.18) 

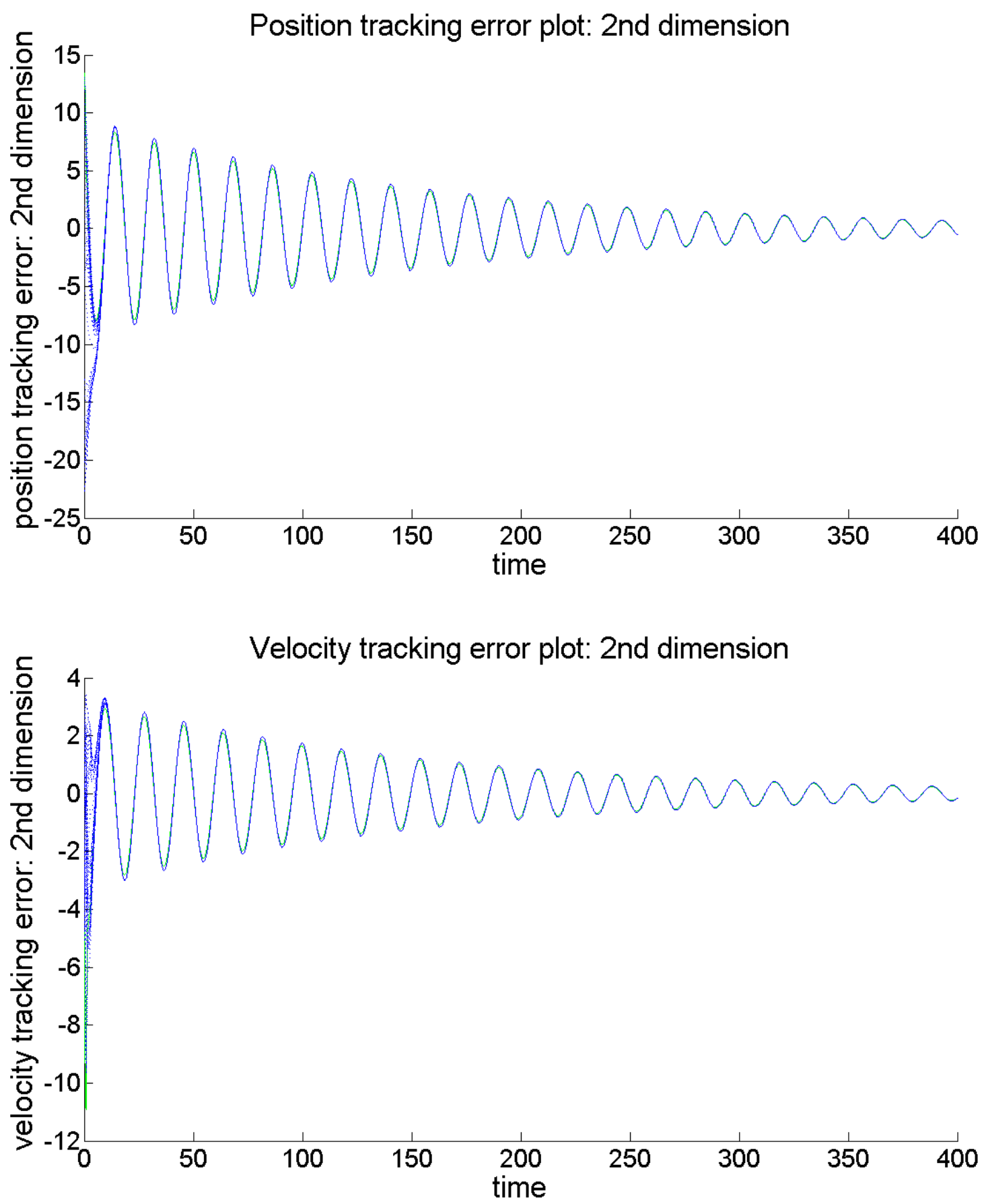

Figure 5.6: The 2nd dimension tracking errors between follower agents and the virtual leader under control protocols (5.18)

\subsection{Summary}

In this chapter, we revisited the problem of distributed virtual leader tracking for a class of multi-agent systems with second order agent dynamics under a state dependent dynamic topology. In particular, we 
proposed a controller structure that is much simpler than those in the literature to solve the multi-agent system virtual leader tracking problem under a jointly connected state dependent topology. In addition, we have also proposed a novel connectivity enhancing mechanism in which the adjacency matrix is defined in such a way that the mild topology connectivity condition can be more easily satisfied in real world implementations. In comparison with existing topology preserving algorithms, our proposed mechanism is effective in maintaining both the initially existent topology edges and those newly formed topology edges that were not initially existent, and does not require the initial topology to be initially connected. Therefore, under our proposed mechanism, the topology connectivity could be enhanced as time goes by. In contrast, existing connectivity preserving coordinated tracking control results all assume the interaction topology to be initially connected, and technical issues on solution uniqueness of the closed-loop system have not been adequately addressed. Our proposed technique applies to both consensus tracking and flocking. It is noted that the proposed connectivity enhancing mechanism and the corresponding analysis technique are also applicable to connectivity enhancing coordinated control problems of other multi-agent systems. 


\section{Chapter 6}

\section{Conclusions}

This dissertation studied the distributed coordination control of complex multi-agent networks with dynamic interaction topologies. Both state independent interaction topologies and state dependent interaction topologies have been addressed.

In the first part of this thesis, we solved the distributed synchronization control problem for a multi-agent network with $n$th order unknown nonlinear agent dynamics. We established the results for both an undirected time varying interaction topology and a directed time varying interaction topology. A standard assumption on the interaction topology connectivity in the literature on this type of problems was made, namely, the interaction topology is connected. Distributed synchronization control algorithms were developed and the desired synchronization performance of the multi-agent network system was proven.

In the second part of this thesis, we investigated the distributed consensus control problem of a multi-agent network with high order linear agent dynamics under a directed jointly connected interaction topology. This enriches the existing literature on multi-agent distributed consensus control under a jointly connected directed interaction topology, which was limited to very simple single integrator agent dynamics. The distributed control protocols were developed and the consensus was proven.

In the third part of this thesis, we addressed the distributed coordinated tracking problem for a multi-agent network under a state dependent jointly connected dynamic interaction topology. In many applications such as in situations where information exchange among agents is conducted via equipped sensors, the interaction topology depends on agents' states such as the distance among agents, and cannot be simply assumed as a function of time. The distributed control protocols are developed and the coordinated tracking was proven. In addition, a novel topology connectivity enhancing mechanism is proposed to help ensure coordinated tracking in real world implementations. In comparison with the existing topology connectivity preserving 
algorithms, our proposed topology connectivity enhancing mechanism is effective in maintaining both the initially existent topology edges and the newly formed topology edges, which enables us to relax the more restrictive requirement that the interaction topology is initially connected.

For each of the coordinated control problems considered in this dissertation, simulation results were presented to illustrate the performance of the proposed coordinated control protocols.

For future research, it will be interesting to extend the results in this thesis to also take into account other practical factors such as time delay effects and actuator saturation. Besides, the extension of these results to multi-agent network systems with more general agent dynamics is another promising future research direction to pursue. Finally, extension of the work in this dissertation to scenarios where there are both cooperative and competitive agents would also be an interesting and challenging future research direction. 


\section{Bibliography}

[1] M. H. DeGroot. Reaching a consensus. Journal of the American Statistical Association, 69(345), 118-121.

[2] J.N. Tsitsiklis (1984). Problems in Decentralized Decision Making and Computation. Ph.D. Dissertation. Massachusetts Institute of Technology, Cambridge, MA.

[3] J. N. Tsitsiklis, D. P. Bertsekas, and M. Athans (1986). Distributed asynchronous deterministic and stochastic gradient optimization algorithms. IEEE Transactions on Automatic Control, 31(9), 803-812.

[4] T. Vicsek, A. Czirk, E. B. Jacob, I. Cohen and O. Schochet. Novel type of phase transitions in a system of self-driven particles. Physical Review Letters, 75(6), 1226-1229, 1995.

[5] N. A. Lynch. Distributed Algorithms. San Francisco, CA: Morgan Kaufmann.

[6] A. Jadbabaie, J. Lin and A. Morse (2003). Coordination of groups of mobile autonomous agents using nearest neighbor rules. IEEE Transactions on Automatic Control, 48(6), 988-1001.

[7] R. Olfati-Saber and R.M. Murray (2004). Consensus problems in networks of agents with switching topology and time-delays. IEEE Transactions on Automatic Control, 49(9), 1520-1533.

[8] W. Ren and R.W. Beard (2005). Consensus seeking in multiagent systems under dynamically changing interaction topologies. IEEE Transactions on Automatic Control, 50(5), 655-661.

[9] R. Olfati-Saber(2006). Flocking for multi-agent dynamic systems: Algorithms and theory. IEEE Transactions on Automatic Control, 51(3), 401-420.

[10] R. M. Murray (2007). Recent research in cooperative control of multivehicle systems. Journal of Dynamic Systems, Measurement and Control, 129(5), 571-583.

[11] Z. Hou, L. Cheng, and M. Tan (2009). Decentralized robust adaptive control for the multiagent system consensus problem using neural networks. IEEE Transactions on Systems, Man, and Cybernetics-Part B: Cybernetics, 39(3), 636-647. 
[12] A. Das and F. L. Lewis (2010). Distributed adaptive control for synchronization of unknown nonlinear networked systems. Automatica, 46, 2014-2021.

[13] A. Das and F. L. Lewis (2011). Cooperative adaptive control for synchronization of second-order systems with unknown nonlinearties. International Jonoural of Robust and Nonlinear Control, 21, 1509-1524.

[14] Z. Qu (2009). Cooperative Control of Dynamical Systems: Applications to Autonomous Vehicles. New York: Springer-Verlag.

[15] W. Ren and R.W. Beard (2008). Distributed Consensus in Multi-vehicle Cooperative Control: Theory and Applications. Springer: London, 2008.

[16] J. Hu and Y. Hong (2007). Leader-following coordination of multi-agent systems with coupling time delays. Physica A, 374, 853-863.

[17] Y. Hong, L. Gao, D. Cheng and J. Hu (2007). Lyapunov-based approach to multiagent systems with switching jointly connected interconnection. IEEE Transactions on Automatic Control, 52(5), 988-1001.

[18] P. Lin and Y. Jia (2009). Consensus of second-order discrete-time multi-agent systems with nonuniform time-delays and dynamically changing topologies. Automatica, 45(9), 2154-2158.

[19] P. Lin and Y. Jia (2010). Consensus of a class of second-order multi-agent systems with time-delay and jointly-connected topologies. IEEE Transactions on Automatic Control, 55(3), 778-784.

[20] W. Yu, G. Chen and M. Cao (2010). Distributed leader-follower flocking control for multi-agent dynamical systems with time-varying velocities. Systems \& Control Letters, 59(9), 543-552.

[21] Y. Cao and W. Ren (2012). Distributed coordinated tracking with reduced interaction via a variable structure approach. IEEE Transactions on Automatic Control, 57(1), 33-48.

[22] H. Su, X. Wang and Z. Lin (2009). Flocking of multi-agents with a virtual leader. IEEE Transactions on Automatic Control, 54(2), 293-307.

[23] Z. Ding (2013). Consensus output regulation of a class of heterogeneous nonlinear systems. IEEE Transactions on Automatic Control, 58(10), 2648-2653.

[24] Y. Su and J. Huang (2012). Stability of a class of linear switching systems with applications to two consensus problems. IEEE Transactions on Automatic Control, 57(6), 1420-1430.

[25] J.A. Fax and R.M. Murray (2004). Information flow and cooperative control of vehicle formations. IEEE Transactions on Automatic Control, 49(9), 1465-1476. 
[26] R. Olfati-Saber (2006). Flocking for multi-agent dynamic systems: algorithms and theory. IEEE Transactions on Automatic Control, 51(3), 401-420.

[27] F. Xiao and L. Wang (2008). Asynchronous consensus in continuous-time multi-agent systems with switching topology and time-varying delays. IEEE Transactions on Automatic Control, 53(8), 1804-1816.

[28] P. Lin and Y. Jia (2009). Consensus of second-order discrete-time multiagent systems with nonuniform time-delays and dynamically changing topologies. Automatica, 45(9), 2154-2158.

[29] Y. Zhang and Y. Tian (2010). Consensus of data-sampled multi-agent systems with random communication delay and packet loss. IEEE Transactions on Automatic Control, 55(4), 939-943.

[30] C. Ma and J. Zhang (2010). Necessary and sufficient conditions for consensusability of linear multi-agent systems. IEEE Transactions on Automatic Control, 55(5), 1263-1268.

[31] J. Hu and G. Feng (2010). Distributed tracking control of leader-follower multi-agent systems under noisy measurement. Automatica, 46(8), 1382-1387.

[32] R. Carli, A. Chiuso, L. Schenato and S. Zampieri (2011). Optimal synchronization for networks of noisy double integrators. IEEE Transactions on Automatic Control, 56(5), 1146-1152.

[33] Z. Ji, H. Lin and H. Yu (2012). Leaders in multi-agent controllability under consensus algorithm and tree topology. Systems \& Control Letters, 61(9), 918-925.

[34] Y. Su and J. Huang (2012). Cooperative output regulation of linear multi-agent systems by output feedback. Systems \& Control Letters, 61(12), 1248-1253.

[35] G. Guo, L. Ding and Q. Han (2014). A distributed event-triggered transmission strategy for sampled-data consensus of multi-agent systems. Automatica, 50(5), 1489-1496.

[36] H. Du, G. Wen, X. Yu, S. Li and M.Z.Q. Chen (2015). Finite-time consensus of multiple nonholonomic chained-form systems based on recursive distributed observer. Automatica, 62, 236-242.

[37] H. Meng, Z. Chen, L. Zhu and R. Middleton (2015). Consensus of a class of second-order nonlinear heterogeneous multi-agent systems with uniform time delay. Proceedings of the 2015 IEEE Conference on Decision and Control, Osaka, Japan, 2341-2346.

[38] S. Su, Z. Lin, and A. Garcia (2014). Distributed synchronization control of multi-agent systems with unknown nonlinearities: the case of fixed directed communication topology. Proceedings of the 2014 American Control Conference, Portland, Oregon, USA, 5361-5366. 
[39] S. Su and Z. Lin (2015). On distributed consensus control of higher-order systems with dynamically changing directed interaction topologies. Proceedings of the 2015 34th Chinese Control Conference, Hangzhou, Zhejiang, China, 7102-7107.

[40] S. Su and Z. Lin (2015). Distributed synchronization control of multi-agent systems with switching directed communication topologies and unknown nonlinearities. Proceedings of the 2015 IEEE Conference on Decision and Control, Osaka, Japan, 2341-2346.

[41] S. Su and Z. Lin (2016). Distributed consensus control of multi-agent systems with higher order agent dynamics and dynamically changing directed interaction topologies. IEEE Transactions on Automatic Control, 61(2), 515-519.

[42] S. Su, Z. Lin, and A. Garcia (2016). Distributed synchronization control of multi-agent systems with unknown nonlinearities. IEEE Transactions on Cybernetics, 46(1), 325-338.

[43] S. Su and Z. Lin. A Multiple Lyapunov function approach to distributed synchronization control of multi-agent systems with switching directed communication topologies and unknown nonlinearities. IEEE Transactions on Control of Network Systems, DOI: 10.1109/TCNS.2016.2570016.

[44] S. Su and Z. Lin (2017). Distributed virtual leader tracking of multi-agent systems with second order agent dynamics under a state dependent jointly connected topology. Proceedings of the 2017 American Control Conference, Seattle, Washington, USA, 2014-2019.

[45] S. Su and Z. Lin. Connectivity enhancing coordinated tracking control of multi-agent systems with a state-dependent jointly-connected dynamic interaction topology. Automatia, under review.

[46] S. Su, Y. Wei and Z. Lin, "Stabilization of Discrete-time Linear Systems with an Unknown Time-varying Delay by Switched Low Gain Feedback," IEEE Transactions on Automatic Control, under review.

[47] X. Jin. Adaptive iterative learning control for high-order nonlinear multi-agent systems consensus tracking. Systems \& Control Letters, 89, 16-23.

[48] R. Olfati-Saber, J.A. Fax and R.M. Murray (2007). Consensus and cooperation in networked multi-agent systems. Proceedings of the IEEE 2007; 95:215-233.

[49] Y. Chen, J. Lu, X. Yu and D. Hill (2013). Multi-agent systems with dynamical topologies: consensus and applications. IEEE Circuits and Systems Magazine, 13(3), 21-34.

[50] Y. Cao, W. Yu, W. Ren and G. Chen (2013). An overview of recent progress in the study of distributed multi-agent coordination. IEEE Transactions on Industrial Informatics, 9(1), 427-438. 
[51] D. Liberzon (2003). Switching in Systems and Control. Cambridge, MA: Birkhuser.

[52] J. Park and I.W. Sandberg (1991). Universal approximation using radial-basis-function networks. Neural Computation, 3, 246-257.

[53] J.-J. E. Slotine and W. Li (1991). Applied Nonlinear Control. Prentice Hall, Upper Saddle River, NJ.

[54] A. Berman and R. J. Plemmons (1979). Nonnegative Matrices in the Mathematical Sciences. Academic Press, Inc., New York. 